\title{
Gheirat as a Complex Emotional Reaction to Relational Boundary Violations:
}

\section{A Mixed-Methods Investigation}

\author{
Pooya Razavi ${ }^{1}$, Hadi Shaban-Azad ${ }^{2}$, Sanjay Srivastava ${ }^{1}$ \\ ${ }^{1}$ University of Oregon; ${ }^{2}$ University of Tehran
}

Forthcoming in the Journal of Personality and Social Psychology 


\begin{abstract}
Author Notes
We gratefully acknowledge the assistance of Marziye Vafayi, Fatima Etemadi, and Sana ShabanAzad in transcribing the interviews. We also thank Arman Amiri and Ahmad Nourmohammadi for assistance in data collection.

Portions of the findings in this article have been presented at meetings of the Society for Personality and Social Psychology (February 2019, Portland, OR; February 2020, New Orleans, LA; February 2022, San Francisco, CA, United States), Society for Affective Sciences (March 2019, Boston, MA, United States), and Association for Research in Personality (July 2021, virtual conference).

Materials, quantitative data, R scripts, and analyses output files required to reproduce the results reported in this article are available at https://osf.io/pxsbv/

Correspondence concerning this article should be addressed to Pooya Razavi, Department of Psychology, University of Oregon, 1227 University St, Eugene, OR 97403; email: pooyar@uoregon.edu

(C) 2022, American Psychological Association. This paper is not the copy of record and may not exactly replicate the final, authoritative version of the article. Please do not copy or cite without authors' permission. The final article will be available, upon publication, via its DOI: $10.1037 / \mathrm{pspp} 0000424$
\end{abstract}

\title{
APA Citation:
}

Razavi, P., Shaban-Azad, H., \& Srivastava, S. (2022). Gheirat as a complex emotional reaction to relational boundary violations: A mixed-methods investigation. Journal of Personality and Social Psychology, doi: 10.1037/pspp0000424 


\begin{abstract}
People from different cultural backgrounds vary in how they define, perceive, and react to violations of relational boundaries. Muslim cultures are diverse and include nearly one in four people in the world, yet research on their relational and moral norms is scarce. We contribute to narrowing this gap by studying gheirat, a moral-emotional experience ubiquitous in Muslim Middle Eastern cultures. In four mixed-methods studies, we study how gheirat is experienced, what situations elicit it, and its social functions among Iranian adults $(N=1107)$ using qualitative interviews, scenario- and prototype-based surveys, and an experiment. The prototypical experience of gheirat consisted of diverse appraisals (including sense of responsibility, insecurity, and low self-worth) and emotional components (including hostility, social fears, and low empowerment). We identified three types of relational violations that elicit gheirat: harm or insult to namoos (people and self-relevant entities one is obliged to protect), romantic betrayal by namoos, and intrusions by a third person. Each violation type led to a distinct variant of the prototype. Contrary to folk theories of gheirat, we did not find support for the idea that gheirat is a predominantly male experience. However, an experiment on the signaling effects of gheirat revealed that gheirat-expressors are ascribed both positive and negative traits, but positive traits prevail for men and negative traits prevail for women. We discuss how the results contribute to a better understanding of Iranian social life and intercultural contact, as well as the implications for theories of emotion and the cultural logic of honor.
\end{abstract}

Keywords: culture, moral emotions, relational boundaries, person perception, emic-etic 
I was at a party with my wife. She was wearing a form-fitting dress... while we were dancing, I noticed that other men are checking her out, looking at her body. It took a lot of effort to control myself while we were dancing, but afterwards I told my wife that her dress is inappropriate, and did not allow her to dance again.

$$
\text { Male - } 33 \text { years old }
$$

We were walking in the mall. I saw from a distance that a girl, sitting on a bench, is leering at my husband... I gave her angry looks from afar till we got near her. I kept staring at her until we got past her.

\section{Female - 36 years old}

The first time I saw an Iranian actress's private sex tape; I don't remember her name, but everyone knew her as Zxxxx. I was upset for a few days, and felt stressed, and was sad.

$$
\text { Male-32 years old }
$$

An old street peddler came into the store and the owner reacted inappropriately and threw him out. I reacted by strongly scolding the store owner and refusing to buy anything from him. Then I talked to the peddler and bought some stuff from him.

\section{Female - 33 years old}

The above narratives were provided by Iranians who were asked to write about a time they experienced gheirat $^{l}$. In Iran, gheirat is an important concept in various domains of social life, including romantic relationships, family dynamics, and politics. People often refer to experiencing or expressing gheirat when there is a violation involving people or entities toward whom a person feels a strong connection and a tendency to protect. As illustrated in the example

\footnotetext{
${ }^{1}$ Multiple transliterations of this word exist, including qeirat, ghayrah, ghira, ghayrat, gheerah, and gheera.
} 
narratives above, the violations that lead to the experience of gheirat are surprisingly diverse, ranging from violations of socio-sexual norms to mistreatment of the vulnerable. Similarly, the emotional experiences associated with gheirat are heterogeneous, ranging from jealousy, to sadness, distress, and moral outrage. It is perhaps due to these complexities that, in the absence of an English equivalent for the word gheirat, contiguous constructs such as jealousy, protectiveness, intrasexual rivalry, honor, or confrontativeness are sometimes used to describe gheirat to Western audiences (see Atari \& Jamali, 2016). In the present research, we applied a mixed-method approach (Schoonenboom \& Johnson, 2017) to our investigation of gheirat among Iranian adults with the goal of generating and investigating theories that shed light on the antecedents, emotional, cognitive, and social consequences associated with this important phenomenon.

\section{Why study gheirat? Rationale and approach}

Among Iranians, gheirat is a ubiquitous concept which appears in news, arts, and daily conversations about romantic and familial relations (Allamezade, 2016; Tabatabaei, n.d.), domestic politics (“Mousavi’s delusion”, 2011; Torabifard, 2020), international relations and conflicts (Bastani, 2016; “The gheirat and courage”, 2013), and even the Iran's nuclear program and negotiations with world powers (Behravesh, 2015; “Abedi: Nuclear technology”, 2011; “About Majid Shahriari”, 2019). The norms related to gheirat are grounded in Islamic teachings, and similar examples can be found in other Muslim cultures of the Middle East (AzarbaijaniMoghaddam, 2012; Iqbal, 2012; Malik, 2018; Öner, 2018). Gheirat's relevance to such a diverse set of value-laden experiences points to its importance as a "key cultural concept" (Bakhtiar, 2015; Wierzbicka, 1997) that can provide insight into the cultural norms and psychological 
dynamics of one of the most understudied populations in psychology (Salari Rad et al., 2018; Veillard, 2017).

From a theoretical perspective, studying gheirat contributes to our broader understanding of morality, emotions, and culture. Psychology's general dependence on samples from a small slice of the human population has led to theories that may lack validity, generalizability, and interpretive power when applied to non-represented populations (Brady et al., 2018; Medin et al., 2017). This is especially pertinent to the fields of emotion and morality in which questions about universality and cultural-specificity can directly influence the core assumptions of the field (Ekman \& Cordaro, 2011; Haidt et al., 1993; Mesquita \& Boiger, 2014; Russell, 1991a; Shweder, 1982; Wierzbicka, 1986) and where investigations in new cultural settings have challenged tenets of theories previously accepted as universal (e.g., Miyake et al., 1985; Snarey, 1985; Takahashi, 1990). A rigorous investigation of gheirat, its antecedents and consequences, and its social function can contribute to cumulative theories of how culture shapes emotions.

Furthermore, research on gheirat can have direct applications for contexts in which those associated with Muslim and non-Muslim cultural backgrounds interact. In the absence of an understanding of the cultural processes that influence others' motivations, people are prone to interpret outgroups' actions as "irrational, deficient, or even immoral” (Markus, 2016, p. 162), a dynamic that is costly for inter-cultural contact. A better understanding of this key cultural concept can offset that.

To meet these aims, we adopted a combined emic-etic approach. Emic research avoids imposing theories and concepts developed in other cultures, and instead looks at a phenomenon "through the eyes of the people in a particular culture" (Berry, 2013, p. 58). We therefore began with a qualitative study (Study 1), which allowed us to minimize assumptions about the meaning, 
manifestation, and relevance of gheirat and generate theories and hypotheses grounded in the cultural dynamics of the target population. In subsequent studies, we increasingly complemented this emic approach with an etic one by bringing in established theoretical frameworks that were relevant to gheirat. In Study 2, we extended the insights from the qualitative thematic analysis using a prototype approach that drew in part on established models of emotion components. Using a survey of potentially gheirat-eliciting scenarios (Study 3) and a preregistered experiment (Study 4), we addressed important questions about the types of violations that elicit gheirat, the reputational costs and benefits of experiencing and expressing gheirat, and the gender dynamics influencing these processes. By beginning with data-driven description and then moving to hypothesis-testing experimentation (Rozin, 2001; Scheel et al., 2021), we develop a framework for understanding gheirat in relation to current theories of emotion and culture.

\section{Cultural and Empirical Perspectives on Gheirat}

Muslim religious texts constitute the earliest writings on gheirat. These sources are important because in most Muslim-majority countries religion plays a key role in different aspects of the social ecology such as the law (Coulson, 2017), family structure (Dhami \& Sheikh, 2000), and gender norms (Hassan, 1995; Shaheed, 1986). Classical texts do not provide a complete or current understanding of gheirat, but they are an important starting point.

To understand gheirat, it is important to grasp the concept of namoos. Namoos includes a diverse range of people or entities that one feels a sense of connection towards and has an obligation to protect. In many cases, people's narratives about namoos refer to specific individuals, such as romantic partners and family members (especially female kin). However, collectives (such as a tribe or country) and abstract concepts (such as a gender category) may 
also be referred to as namoos (e.g., "The martyr Fahmideh", 2021; Hassanzadeh, 2020; Noori Ala, 2013). Situations that elicit gheirat typically involve namoos in some way.

In both Shi'a and Sunni Islamic texts, gheirat is referred to as a necessary virtue of the pious Muslim (Al-Bukhari, 1976; Mazaheri, 2016; Tabatabaei, 1976). A devout Muslim man is described as someone who is prone to gheirat and reacts accordingly in response to norm violations involving their female namoos (Akbari \& Tetreault, 2014; Motahhari, 1968). Such violations are often concerned with socio-sexual norms (e.g., men approaching someone’s partner or female relatives with sexual intentions) or norms related to chastity (e.g., a female relative dressing or acting in a way that might be perceived as cues of promiscuity; Motahhari, 1968). Importantly, discussions of gheirat are often intertwined with rules about hijab and female dress code. A man who is prone to gheirat is required to be sensitive to how his female relatives present themselves to the society, and if needed, should enforce religious norms related to female dress code (Shirazi, 2004). Many religious sources go beyond these socio-sexual norms and describe gheirat as a broader response to general moral violations. A man who is not gheirat prone is referred to as bigheirat or dayouth (both derogatory terms) and is often depicted as unworthy, incapable of distinguishing right and wrong, and having a weak moral character (Noori, 1987).

While informative, the religious literature on gheirat is mostly prescriptive - it provides guidelines for the ideal, not actual, norms of behavior. From a more descriptive perspective, a limited number of studies of gheirat exist. A linguistic investigation of gheirat (Bakhtiar, 2015) suggests that gheirat is an "emotional alarm system" monitoring the status of the extended self against threats to one's values. Once a threat is detected, often in the form of dangers to reputation, family, religious values, or country, gheirat is experienced, sometimes as a 
combination of emotions such as anger, moral outrage, jealousy, and hatred. From this perspective, gheirat is at the center of a hyper-cognized network of emotional and value-laden concepts such as romantic jealousy, outrage, chivalry, and aberu (face or public image).

Studies on mate preference and retention strategies in Iran corroborate the key cultural role of gheirat. Atari and Jamali (2016) found that Iranian women perceive gheirat-proneness as a favorable characteristic of a potential mate, almost as important as being physically attractive. Iranian adolescents consider gheirat proneness as a character strength alongside purity and chastity (Jabbari et al., 2021). There are also indications that gheirat-related behavior and values have similarities to the dynamics previously studied in other honor cultures. For example, gheirat-prone men are more likely to engage in mate retention tactics such as intrasexual threats and concealment of mate from rivals (Atari et al., 2017), themes that are commonly associated with protection of honor (Atari, 2018; Hupka, 1991). Furthermore, Atari et al. (2020) found a cluster of gheirat-related attitudes (such as vigilance about a romantic partner's clothing or social interactions) to be moderately correlated with, but distinct from honor values (Novin \&

Oyserman, 2016).

\section{Theoretical Frameworks Relevant to Gheirat}

The religious and empirical literature suggests that gheirat is culturally distinct in many ways, but it also may have some similarities with phenomena found in other cultures. In this section, we discuss potential connections to established theories of emotion and culture, which influenced the present program of research.

Gheirat as a multi-component emotional experience. Gheirat is described as an emotional experience with diverse manifestations (Bakhtiar, 2015; Tawasil, 2015). For example, in the absence of a direct translation, gheirat is sometimes described as a kind of jealousy (e.g., 
Shahabi \& Etaf, 2014; Barakatullah, n.d.)). The similarities between certain characteristics of romantic jealousy and gheirat are clear: Both play a key role in the mate retention process (Atari et al., 2017; Pfeiffer \& Wong, 1989). Furthermore, jealousy, like gheirat, often manifests as a complex set of emotions with distinct communicative patterns (Guerrero, \& Andersen, 1998). For example, White and Mullen (1989) discuss three basic emotion clusters associated with the experience of jealousy: an anger cluster (e.g., hate and contempt), a sadness cluster (e.g., hopelessness and depression), and a fear cluster (e.g., anxiety and distress). Despite these similarities, there are critical aspects of gheirat that do not fit the jealousy conceptualization. First, even though gheirat towards one's partner is common, the target of gheirat goes far beyond that, and includes people and entities that are not involved in the mating process. Second, even when gheirat is towards the partner, many narratives, as discussed by Bakhtiar (2015), are about protection against harm and do not happen as a result of a romantic rivalry. Third, the social construction and consequences of these concepts differ-gheirat is, at least according to religious texts, often discussed as a virtue, unlike jealousy which is generally perceived negatively (Motahhari, 1968).

In the present research, instead of assuming that gheirat is the same as, or a variant of, another emotion, we started by asking a broader question: "What is gheirat?" We were informed by the theoretical perspective that treat emotional experiences as multi-component processes (for a review, see Lange \& Zickfeld, 2021). From this perspective, emotions involve several components, including appraisals, feelings, somatic experiences, and expressive behavior (Izard, 2010; Moors, 2009; Scherer, 2009). Not all emotions differ on all components, leading to fuzzy boundaries and conceptual overlaps (Russell \& Fehr, 1994; Ellsworth \& Scherer, 2003). As such, a comprehensive examination of multiple components is an effective way to describe an 
emotional experience. Following this perspective, in Study 2, we used a prototype approach to study gheirat and its components.

Cultural logic of honor. Iran, and a number of other predominantly Muslim cultures, are considered to be honor-oriented societies (Aslani et al., 2016; Estiri, 2019; Khurshid, 2012; Said, 1991; Smith et al., 2021). Although the present program of research is not a study of honor or cultural logic of honor, our review identified some relevant theories and findings that informed our work.

Theories of the origins of cultures of honor posit that under specific environmental conditions such as resource scarcity and an absence of strong legal institutions, certain cultural norms become beneficial for the person and adaptive for the survival of the collective (Nowak et al., 2016; Schneider, 1971; Shackelford, 2005; Vandello \& Cohen, 2004). These norms include an emphasis on upholding a reputation as someone who abides by moral rules and does not tolerate transgressions (Nisbett \& Cohen, 1996). Violations can harm a person's social standing as an honorable person, and may even affect a person's survival through mechanisms such as exclusion from the collective or physical harm (Leung \& Cohen, 2011; Thrasher \& Handfield, 2018). Therefore, threats to a person's honor often elicit strong negative emotional reactions (e.g., shame and anger; Rodriguez Mosquera et al., 2008), and elevation of one's honor can lead to positive emotions (e.g., pride; Uskul et al., 2014). Furthermore, individuals or groups that engage in honor-threatening behavior may face aggressive reactions. The goal of such reactions is often two-fold: taking revenge against the transgressor while signaling to others that future violations will be costly, and restoring personal and/or collective honor (Abu-Lughod, 1999; Ashokkumar \& Swann, 2022; Thrasher \& Handfield, 2018). 
Our review of the anecdotal and scholarly writings on gheirat and the literature on honor indicated noteworthy similarities between instances of honor violation (e.g., threats or insults to one's family) and some of the situations that elicit gheirat, suggesting the possibility that gheirat is an emotional response to violations of honor norms that are common in Muslim cultures. For that reason, our investigation was informed by research on the cultural logic of honor. For example, research in honor-oriented cultures demonstrate that honor-threats elicit strong, and sometimes complex, emotions (Rodriguez Mosquera et al., 2002; Uskul et al., 2014). Thus, we investigated a wide range of emotional reactions associated with gheirat and its different elicitors in Study 2. Furthermore, concerns about reputation and social standing are among the central motives driving reactions to honor threats (Nisbett \& Cohen, 1996; Günsoy et al., 2020a). In line with this notion, we investigated the role of concerns for reputation in elicitation and expression of gheirat in Studies 1 and 2, and expand on reputational consequences of expressing gheirat in Study 4. Finally, similar to gheirat, many honor-related norms and behaviors are intertwined with gender norms (Baboli \& Karimi-Malekabadi, 2020; Barnes et al., 2012; Barnes et al., 2014; Rodriguez Mosquera et al., 2002). As such, in all studies, we pay extensive attention to gender dynamics in the experience of gheirat.

Although we were informed by this literature, we were also cautious about constraining our investigation of gheirat by imposing models developed in other cultures onto this new context. There are important cultural differences in conceptualizations and manifestations of honor (Cross et al., 2014; Helkama et al. 2013; Guerra et al., 2013; Günsoy et al., 2020b; Uskul et al., 2012), and as Uskul et al. (2019) note, "honor cultures in different parts of the world such as South Asia, South America, and the Middle East and other parts of Europe [other than southern and south-eastern European honor cultures] have received less attention" in honor 
research (p. 40). Moreover, gheirat may have important features that we did not want to assume, a priori, could be subsumed within an honor framework. Norms that are at the center of the experience of gheirat are rooted in Islamic ideology and drastically differ from norms in many other non-Muslim honor cultures, and honor researchers have observed that theoretical perspectives that are disengaged from the lay conceptions of a phenomenon "risk being overly narrow and neglecting key elements of the phenomenon" (Cross et al., 2014, p. 247). For example, there has been considerable attention to insult and harm as triggers of honor concerns, and aggressive tendencies as the common response to such violations (e.g., Cohen et al., 1996; IJzerman et al., 2007). As demonstrated in one of the participant narratives above, gheirateliciting situations do not necessarily involve direct insult or harm to the self or someone close to the self. They may lead to avoidance-oriented responses in which, instead of engaging with others aggressively to defend one's reputation, the person facing a violation withdraws from the situation and wallows in feelings of lower self-worth and anxiety. To understand these variations in gheirat-eliciting situations, their appraisals, and their emotional consequences, we adopt a combined emic-etic approach (Rodriguez Mosquera, 2018; Arshad \& Chung, 2022) that, while informed by the research on other honor cultures, is culturally and methodologically sensitive and designed to reflect the perspectives of the people who experience gheirat.

\section{Cultural Context}

For readers who are less familiar with Iran, we provide a brief discussion of some of the geographic, historical, and cultural characteristics that have influenced the social lives of Iranians and may be relevant to the present work. Iran is located in the southwest region of the Asian continent. With a population of approximately 82 million people ( $4.6 \%$ of the world's population), it is the seventh most populous Muslim-majority country and home to the largest 
Shi'a Muslim population in the world (Pew Research Center, 2009). Iran is a multi-ethnic country (e.g., Fars, Turk, Kurd, Lor) where the official language of education is Farsi/Persian. Other languages and dialects (e.g., Azerbaijani, Kurdish, and Luri) are spoken by many Iranians (Moradi, 2020; Windfuhr, 1991). Iran is considerably different from its neighbors and the countries in the region in terms of the ethnic composition of the population, spoken languages, GDP, political system of governance, and international relations. For example, Iran's GDP per capita in 2020 was $\$ 2422$, considerably lower than Turkey (\$8536) and Saudi Arabia (\$20110) and higher than Pakistan (\$1189) and Afghanistan (\$517; The World Bank Data, 2020).

Several historical events are noteworthy for their relevance to the cultural dynamics of contemporary Iran and the role of gheirat. Many Iranian cultural traditions are influenced by Iran's history prior to the advent of Islam. For example, the Iranian New Year (Nowruz) and many social rituals surrounding it are attributed to pre-Islamic Zoroastrian traditions (TavakoliTarghi, 1999; Stausberg et al., 2015). During the centuries after the Muslim conquest of Persia in $650 \mathrm{CE}$, this pre-Islamic cultural heritage (including the Persian language) was heavily influenced by the Islamic and Arab cultural traditions. It has been proposed that concepts such as gheirat were adopted during this historical period. Although for most of its history, Iran (formerly referred to as Persia) has been a monarchy, a popular uprising in 1978-1979 led to the establishment of an Islamic Republic. Post-revolution Iran went through important sociopolitical changes. The new constitution heavily emphasizes the role of religious rules in governance and in social life. Consequently, Islamic rules, such as mandatory hijab and restrictions on contact between men and women, have a strong presence in everyday life and are an important topic of societal and political discourse (Farazmand, 1995; Keddie, 1980). 


\section{Present Research}

We started our investigation with a fully exploratory and descriptive methodology that made as few assumptions about the nature of gheirat as possible. In Study 1, using qualitative methodology, we investigated Iranians' conceptions of gheirat, its situational elicitors, and the cognitive and emotional reactions associated with it. In Study 2 we used a prototype approach to investigate the emotional clusters and cognitive evaluations associated with the experience of gheirat, as well as the role of gender and religiosity. Importantly, the first two studies identified three major situational elicitors of gheirat and their unique emotional and cognitive profiles. In Study 3, we presented participants with a large number of gheirat-eliciting scenarios to further study the types of violations that lead to the experience of gheirat. Furthermore, we investigated how ingroup vs. outgroup membership, gender, and religiosity influence the experience and intensity of gheirat. Finally, in Study 4, using a preregistered experimental design, we investigated how expressing gheirat elicits moral and affiliative judgements that can contribute to one's reputation.

\section{Study 1}

Before we inquire into origins and functional relations, it is necessary to know the thing we are trying to explain (Asch, 1952/1987, p. 65).

Qualitative research offers methodological and analytical depth that is suitable for exploring under-investigated phenomenon (Creswell, 2013; Levitt, 2021) or historically underrepresented populations in the research literature (e.g., The APA Presidential Task Force on Immigration, 2013) and generating theories that can be further tested using quantitative methods (Auerbach, \& Silverstein, 2003; Krauss, 2005). We conducted semi-structured interviews with a heterogenous group of subjects and analyzed the data using thematic analysis (Braun \& Clarke, 
2006). We adopted this approach to generate a rich description of gheirat as it is experienced by Iranians, and to detect patterns to help generate hypotheses regarding the nature of gheirat, its elicitors, and its psychological components. In the broadest sense, our overarching question was, "What is gheirat?" More specifically, the objective was to gain insight into (a) the situations that elicit gheirat, (b) the targets towards which gheirat is experienced, and the behavior towards these targets, (c) the phenomenology of gheirat (i.e., the range of emotional and cognitive experiences during an episode of gheirat), and (d) participants' attitudes towards gheirat and its consequences, and the way the participants interpret gheirat or make sense of it.

For readers less familiar with qualitative methods, we point out two distinct features of a qualitative approach (more details are provided in the Online Supplement). First, statistical generalization to a population is not the primary goal of this approach (Levitt et al., 2018; Norman, 2017). Instead, qualitative methods provide the means to discover and explore variation in the experience of a phenomenon. As such, qualitative generalization is "not to the population but to the phenomenon" (p. 95, Levitt, 2021). Second, the process of data collection and analysis is non-linear and iterative (Levitt et al., 2018; Merriam \& Tisdell, 2015). Sometimes data collection and analysis might overlap, or the researcher may move back and forth between different phases of research to make sense of inconsistencies or reorganize the interpretation of patterns observed in the data (Braun \& Clarke, 2006).

\section{Method}

\section{Ethics Statement}

All APA ethical guidelines, including informed consent, were followed in all data collection. Study 1 was initiated, and its data were collected and analyzed, by the second author in Iran, where non-biomedical psychology research does not require institutional review. The 
first author only worked with fully de-identified data, and the third author did not analyze the qualitative data. Studies 2 to 4 were approved by University of Oregon's IRB (protocol \# 10252016.031, titled "Exploring the psychological elements of "gheirat" among Iranian adults").

\section{Sources of Data}

Researchers. The interview outline and questions for this study were designed by the first two authors. These authors grew up in Iran and have experience of living within and outside Iran. Having been exposed to non-Muslim cultures in Southeast Asia, Europe, and North America, the authors were conscious of the drastic differences among these cultures and the uniqueness of the gheirat experience among Iranians. The authors' perceptions and attitudes about gheirat prior to this research were primarily informed by their lived experiences. Prior to the study, both authors considered gheirat to be a multi-dimensional phenomenon which can manifest in both desirable (e.g., protectiveness towards the vulnerable) and undesirable (e.g., relational aggression following jealousy) forms. The authors were also aware of the potentially harmful biases which can result from cultural essentialism or preconceptions rooted in perceiving all members of a cultural group as psychologically homogeneous (e.g., uncritically interpreting or situating Muslim individuals' behavior within an honor framework; Grewal, 2009; Mayeda \& Vijaykumar, 2016; Shier \& Shor, 2016). To minimize the influence of their personal attitudes on the research process, from the beginning stages of the research process the authors actively sought diverse perspectives about gheirat through collecting different anecdotal and scholarly writings in Persian, Arabic, and English. Furthermore, through open-ended interview questions and heterogeneous sampling, the investigators actively searched for data that disconfirmed their expectations and updated the data collection process accordingly. For example, consistent with the popular cultural belief that gheirat is primarily experienced by men (Khezr-Heidari, 2018), 
prior to this study the authors thought that the interviews should be focused on male participants. Nevertheless, early in the data collection process they interviewed a female participant, after which it became clear that the presupposition about gheirat being a male experience is not accurate. Therefore, the rest of the interviews and analyses were revised so that both male and female perspectives were included. The third author was not involved in the data collection and analysis. Once the initial findings from the thematic analysis were summarized, the third author provided extensive feedback on the coherence of the themes, alternative interpretations of the data excerpts, and recommendations for organizing and presenting the findings.

Participants. Participants were selected from volunteers who reached out to the second author in response to social media announcements (on Facebook and Telegram) regarding a study about the lived experiences of gheirat. Our selection strategy was maximum variation sampling (Palinkas et al., 2015; Patton, 1990), which was informed by the goal to collect a heterogeneous sample that helps cover diverse gheirat-related experiences and strengthens generalizability to the phenomenon. We identified five dimensions of variation (i.e., relationship status, religiosity, socio-economic status, education, and the city in which the participants grew up) critical to constructing a holistic understanding of gheirat. We kept track of variability in these dimensions through the pre-interview conversations and voluntary disclosures during the interviews, and selected volunteers who increased heterogeneity.

The sampling procedure was iterative and interwoven with the analysis process. We used thematic saturation as the criteria for sample size sufficiency (Saunders et al., 2018). Some guidelines suggest that thematic saturation (i.e., the point at which no new themes are detected in the data) is reached with approximately 12 to 15 in-depth interviews (Guest et al., 2006). Since the variability in interview content and topic may affect saturation, our plan was to primarily rely 
on regular monitoring in determining the stopping point. Specifically, we began with the goal of interviewing 10 participants from diverse backgrounds. After reaching this goal, an initial analysis was conducted, and tentative themes were produced. We then continued interviews until we reached thematic saturation at the 15th interview (Ando et al., 2014; Lowe et al., 2018) such that the additional data was not contributing to the discovery of new themes. At this point, we continued with four more interviews to ensure that our judgement of thematic saturation is accurate. The final sample consisted of 19 Iranian residents who were on average 29.68 years old $\left(S D_{\text {age }}=3.09\right)$ and consisted of 12 men and seven women. The selected sample included participants that varied in terms of relationship status (single $(n=6)$, in a monogamous relationship $(n=7)$, married $(n=4)$, and in non-monogamous relationships $(n=2))$, religiosity (ranging from conservative Muslim to atheist), socio-economic status (self-identified as low $(n=$ $6)$, middle $(n=11)$, and high $(n=2)$ ), education (high school diploma or pre-university $(n=4)$, undergraduate students or bachelor's degree $(n=5)$, graduate students or graduate degree $(n=$ $10)$ ), and the cities in which they grew up (Amol, Bandar Abbas $(n=2)$, Bandar Anzali, Boroujen, Boushehr, Isfahan, Kabul $(n=2)$, Kerman, Kermanshah, Khoi, Rasht, Roudaan, Shiraz $(n=3)$, Tehran $(n=2))$. Participation in this and the rest of the studies did not involve any monetary incentive or compensation.

\section{Procedure and Materials}

This study was not preregistered. The data were collected through semi-structured indepth interviews (DiCicco-Bloom \& Crabtree, 2006; Jamshed, 2014) which were conducted and analyzed over a period of 13 months. Consistent with general guidelines for semi-structured interviews (Corbin \& Strauss, 2008; Merriam \& Tisdell, 2015), the first and second authors developed five over-arching open-ended interview questions that corresponded with the 
previously mentioned objectives of the study. The initial questions were piloted once and refined to improve depth and clarity. The final questions (see the Online Supplement for the exact wording) provided a general structure for the interviews. Respondents were asked to talk about (a) a time they experienced gheirat, (b) the people they usually experience gheirat towards, (c) the kind of events or experiences that leads to the experience of gheirat, (d) psychological and phenomenological aspects of their experience of gheirat (e.g., cognitive appraisals, behavioral reactions, etc.), and (e) the effect gheirat has had on different aspects of their daily life (e.g., romantic or family relationships, friendships, etc.). Within this structure, when needed, the interviewer explored the respondents' answers by probing and asking specific questions that helped extend or clarify the initial responses. All interviews were in-person and were conducted by the second author in Persian/Farsi language. They took between 23 to 78 minutes $(M=45, S D$ =17). Prior to analyses, verbatim transcripts of all interviews were created $(M=6580$ words; $S D$ $=2958)$.

\section{Analytic Process}

The analytic process was consistent with the widely accepted guidelines for thematic analysis by Braun and Clarke (2006): First, the researchers familiarized themselves with the data through repeated listening of the audio and reading of the transcripts. Second, the interviews were reduced to initial codes which included the most basic meaningful elements of the raw data (Boyatzis, 1998). Third, the initial codes were sorted into potential themes. This was an iterative process, with the main goal of combining the consistent codes into broader themes or subthemes. In the case of disagreement, the authors would discuss and explain their decision process extensively, revisit the interview transcripts, and reevaluate the extracted codes in an iterative process until they reached consensus. Fourth, the themes created in the previous phase were 
reviewed to make sure they are internally homogeneous, and externally heterogeneous (Patton, 1990). As a result, some themes were consolidated, some were broken into several themes, and a few potential themes were removed because the data to support them were not sufficient.

\section{Results}

Consistent with the APA reporting guidelines (Levitt et al., 2018), we provide data extracts related to each theme directly quoted from the interviews.

\section{Concerns that Led to Elicitation of Gheirat}

When participants discussed situations where they, or someone they knew, experienced or expressed gheirat, there was often at least one of these concerns present:

(a) Perception of harm or insult. This was the most consistent concern discussed by all participants. The situation often included a potential harm or insult to someone who is close to the participant, someone who is perceived as vulnerable, or an entity that is perceived as sacred or pure. Once the participant observed or anticipated the occurrence of such harm, they experienced gheirat followed by a need to take action to protect the person being harmed:

“On the street, some dude was harassing a girl, I didn't even know the girl, but I went and told the guy 'why are you bothering her? Who gave you the right?'... I'd like the same thing to happen if someone else is bothering my namoos on the street and I'm not there." -Male, 34 years old.

When talking about harm or insult, participants referred to a wide range of events such as rude comments, verbal insults, sending offensive messages, physically hurting someone, and sexual assault. Importantly, participants referred to verbal insult and physical harm as interrelated violations, a characteristic that is common to this cultural setting and many other honor cultures (Leung \& Cohen, 2011; Maitner et al., 2017). Gheirat was elicited either as a result of 
observing these events, hearing about them happening in the past, or anticipating that such event can happen to namoos. The target of the insult was often described as either vulnerable or sacred, and the participants reported a sense of responsibility to protect the target:

"I experience it towards children too. For example, a woman was hitting her child. It was offensive to me. I wanted to go talk to her... when you see someone is subject to injustice and can't defend themselves, you feel gheirat, [you are] incited to go protect them."Female, 35 years old

(b) Violation of namoos' sacred boundary by a third person. Interviewees talked about a "sacred space," often referred to as harim, that they believed exists around their partner or other close female relatives (e.g., sister and mother); there are boundaries around this space, and only a person's partner, or close relatives are allowed within this space. Participants consistently reported strong feelings of gheirat once an outsider (especially a man) tried to cross this boundary:

"She was the first serious girlfriend I ever had [...] she told me that once she was alone with her lecturer, I think she said that the lecturer either touched her or propositioned her or something like that. I didn't know her well, but I thought the teacher was at fault and wanted to go fight with him, which she stopped me."--Male, 31 years old There was considerable variability in what participants perceived as boundary violations. Examples of such behaviors included looking at, leering, or checking out the namoos, catcalling, dancing or chit-chatting with the namoos, showing interest or trying to establish a relationship. In participants' narratives, there seemed to be an expectation that men should be aware of such boundaries and be careful not to cross them. However, participants expressed concerns that men 
do not always abide by these codes, and this puts women in danger of harassment, or increases the possibility that other men try to establish a relationship with them:

"Maybe I go out, for example, with a top and short pants someday... What will happen? Will someone chase me? Will people give me dirty looks? No!... If a woman goes out with a top and shorts in our society, is that the same as a man going out like that? From the janitor of this apartment complex to people on the streets, all will chase her."-Male, 32 years old

To counter these possibilities, one common strategy expressed by the male participants was to restrict the way their female relatives dress or interact with the opposite sex:

"Nowadays it's more towards my wife, previously it used to be towards my female cousins; I tell them 'wear this, or don't wear that, cover your hair, wear chador.' I try to make the situation so that [when we are] outside, no one says anything to them or looks at them."-Male, 24 years old When describing their experience of gheirat, female participants mentioned being the target of such restrictive behavior:

"For example, your boyfriend might tell you "why did you talk about a certain thing with so and so?" or "don't wear that dress, it's too tight or too short!"-Female, 32 years old While this strategy of influencing namoos's behavior is intended as an anticipatory measure, in the event that someone actually violated the boundary of namoos, male participants reported strong, sometimes aggressive, reactions towards the perpetrators:

"I had a date with my girlfriend. When I arrived there, I saw two boys catcalling her. I don't know what happened, I just know I got to them and beat them up, punching them and kicking them, I destroyed them."-Male, 34 years old 
It is important to note that this theme manifested strongly among the male participants' narratives, raising the possibility that perhaps this type of gheirat is more commonly (or exclusively) experienced by men. This is consistent with the popular accounts that perceive gheirat to be a primarily male mate-retention strategy (e.g., Khezr-Heidari, 2018; Mortazavi \& Poelker, 2017). We will continue investigating the role of gender in the next three studies.

(c) Perceptions of losing namoos. A third theme consisted of situations where the participants felt that namoos (often their partner) wanted to cross the established boundaries of the relationship, which led to the possibility of losing them to a rival. The behavioral elicitors of this type of gheirat were sometimes as subtle as the participants' partner complimenting or paying attention to another person, or as drastic as the partner getting romantically involved with another person. When talking about this type of situations, both male and female participants expressed concerns such as threat of loss, a sense of abandonment, or anticipating the possibility of infidelity as part of their narratives, which had considerable similarities to the experience of romantic jealousy (Radecki-Bush et al., 1993; Sharpsteen, 1993). In fact, some participants had theories about the relationship between jealousy and gheirat:

"I think gheirat sometimes comes out of jealousy, feeling of losing something, and that's why we feel that emotion and we call it gheirat...For example, someone who is the focus of your attention, you don't want him to pay attention to someone else. You want to be the center of his attention and any other attention can bother you and you may react."Female, 30 years old

\section{Targets for which gheirat is experienced}

In describing the targets of gheirat, there was some variability in what constitutes namoos for each interviewee. The most common targets in the narratives included partner, followed by 
first-degree relatives, especially the female ones. Almost all participants expressed a positive relationship between how much they like a person (or they care for them), and the frequency of experiencing gheirat towards them:

"Gheirat is about people who are close and intimate... As the fondness for someone increases, the feeling of protection and care, or even possessiveness increases."-Female, 32 years old

"If you see someone being harassed or harmed on the street, you might be offended; but in many cases you say 'why should I get involved? How is that my business?' But if you are closer [to the person], you have a stronger feeling to defend and protect [them] against harm."-Male, 34 years old

Of the three types of gheirat-eliciting situations discussed above, narratives with the theme of "perception of harm or insult" had the widest range of targets. This was the only category where participants' gheirat was extended to non-related individuals and abstract concepts such as religion, ethnicity, sports team, or even one's career:

"When people talk [negatively] about my nationality, I feel gheirat."-Female, 30 years old

"Sometimes gheirat is about nationality. For example, you go watch a football match, you can't sit there in the corner indifferently...This has to do with gheirat, national pride."Male, 28 years old

\section{Attitudes towards and Perceptions of Gheirat}

Participants often expressed their personal theories about gheirat, as well as how they perceive or evaluate it. Three important sub-themes emerged from these discussions: 
Gheirat as an automatic or regulated reaction. Participants often described their reaction to a gheirat-eliciting situation as automatic and arising unbidden, similar to how many people describe emotions (Averill, 1980). Especially in situations where there was an aggressive reaction to the situation, several participants chose statements such as "I don't know what happened" or "I lost control" to describe the automaticity of their reaction or how overwhelmed they were by their experience of gheirat. Furthermore, several participants referred to gheirat as "an instinct," "unconscious," or "natural" reaction:

"I think gheirat is part of every human's nature and it is a mistake that many people in Iran think that such a thing does not exist in other cultures and it's only a male Iranian thing... gheirat has an instinctive root."-Male, 30 years old

Expressing gheirat to save face. This belief in the automaticity of gheirat, however, does not mean that participants always lost control, or did not regulate their gheirat at all. In fact, some participants discussed upregulating or downregulating their emotional reaction in order to meet societal expectations. For example, male participants reported external societal pressures (e.g., concern for reputation) or the positive impression that expressing gheirat might leave on others as their reason for expressing it:

"When it happens, it's because it's a norm in the society. For example, my sister wears something and I tell her 'don't wear this, we'll lose face!'”- - Male, 30 years old "I had a girlfriend...in her absence, I didn't have much gheirat towards her, but in her presence, I showed myself as someone prone to gheirat, because girls like this feeling of gheirat."-Male, 32 years old

The negative side of gheirat. There was considerable variability in how the participants evaluated gheirat as a moral experience. In contrast to those who saw gheirat as a necessary and 
justified reaction, several participants expressed negative attitudes about some types of gheirat, especially when it led to restricting the behavior of a romantic partner or female relatives:

"I'm not like what I read in some newspapers, for example, brother killed his sister or husband killed his wife; this is not comprehensible for me. Even if I notice that my sister had an extra-marital affair with a stranger, I will never allow myself to even lay a hand on her. If it's the case that she has been raped, I might get so furious that I might go cut the guy into pieces; but if it's consensual, and they are adults who wanted it, what do I have to tell her?"-Male, 31 years old "When gheirat means taking responsibility, that's a good thing. But when it means being controlling and insulting [namoos's] intelligence, that's not good."-Female, 30 years old

Defining and describing gheirat. Even though participants could recount instances when they had experienced gheirat, when asked to provide their definition, some participants (both male and female) found gheirat to be a challenging construct to define, often pointing out the multifaceted nature and meaning of gheirat:

"This gheirat is a very chaotic and hard-to-define word, because it is being interpreted in many different ways in our society." —Female, 23 years old

"What does gheirat mean? [long pause] I'm not sure, it could mean the emotion felt after someone has done something like disrespect, or like being possessive about [namoos's] clothing." —Male, 30 years old

Gheirat as a male experience. A theme common among the female participants was a sense of uncertainty about whether gheirat is an exclusively male experience, or it is qualitatively 
different for men and women, pointing to the importance of gender in conceptualizations of gheirat:

"I never thought about when I might experience gheirat. I always expected that it's a male thing and men feel gheirat."-Female, 32 years old

"I think the same way that there is male gheirat, there is female gheirat... but gheirat in marriage is more common among men than women."-Female, 28 years old

\section{Discussion}

Thematic analyses indicated that there are three types of concerns and situational appraisals that often elicit gheirat, each of which seem to be associated with different emotional responses and cognitive evaluations. We re-examined and extended this finding in Study 2 and 3 using mixed methods and quantitative approaches. Additionally, results demonstrated considerable variability in gheirat frequency and intensity based on the characteristics of the gheirat-expressor and the target of gheirat. Using a large set of gheirat-eliciting scenarios in Study 3, we further investigated the individual differences and cultural backgrounds of the gheirat-elicitor (e.g., religiosity, gender differences), and the characteristics of the norm violator (e.g., ingroup vs. outgroup) or the target of the violation (e.g., male vs female relative) in relation to the frequency and intensity of gheirat. Finally, several participants expressed ambivalent attitudes and judgments about gheirat-proneness and emphasized the importance of protecting one's reputation and saving face as a motive behind regulating their expression of gheirat. We addressed these themes in Study 4, where the multi-dimensional judgments and social consequences of expressing (vs. not expressing) gheirat was studied using a preregistered experimental design. 


\section{Study 2}

In Study 2, our goal was to complement and extend some of the key findings from the previous study using a mixed method prototype-based approach (Rosch, 1975; Russell, 1991b; Shaver et al., 1987). Grounded in the insights from the qualitative interviews and the prior writings on gheirat, we organized our inquiry around three main questions.

\section{Describing the Prototype}

In this study, our first goal was to explore and describe the common properties of a prototypical experience of gheirat. Participants' narratives and discussions pointed to gheirat as a morally-laden emotional experience with diverse situational elicitors and appraisals. The broader literature on the fundamental studies of emotions suggests that emotional experiences often entail multiple components (i.e., affective experience, appraisal of the situation or elicitor, physiological or bodily symptoms, and behavioral responses; Barrett et al., 2004; Moors et al., 2013; Roseman et al., 1994; Scherer, 1984, 2005). During the qualitative interviews, these components were evident in the participants' narratives. For example, interviewees regularly mentioned affective states (e.g., jealousy, anxiety, anger), situational appraisals (e.g., being insulted or betrayed), and diverse behavioral reactions (e.g., withdrawing from the situation, aggressing against the perpetrator) when describing their gheirat experiences. In this study, we extended our understanding of these components using a mixed method prototype approach.

To do this, we asked participants to write about a time they experienced gheirat.

Afterward they made ratings of their affective experiences during this episode, their appraisals of the situation, their bodily and physiological experiences, and their behavioral responses. Understanding the experience of gheirat through examining these components provides insights into the social construction of gheirat (e.g., what kinds of appraisals elicit gheirat?), 
phenomenology of gheirat (e.g., what are people experiencing during a gheirat episode?), and the relation between gheirat and other emotional constructs. Furthermore, such a multicomponent granular description of gheirat allows us to further investigate the systematic sources of variance in the experience of gheirat, such as situational elicitors and gender differences.

\section{Gheirat in Response to Different Elicitors}

During the qualitative interviews, participants showed an awareness of the heterogeneity of different gheirat experiences, especially in terms of the different concerns and situations that lead to gheirat. Our qualitative analysis suggested that while there are commonalities in experiences of gheirat, there seems to be systematic differences depending on the type of violations that elicit gheirat. To expand on this point, our second goal in Study 2 was to investigate the variants of the prototype of gheirat and to discover the features that make these variants distinct from each other (Clore \& Ortony, 1991). This approach has been extensively used to develop theoretical models of emotion categories and is especially fruitful in cases where the emotional experience has "fuzzy" linguistic boundaries (e.g., awe; Keltner \& Haidt, 2003). To do this, we coded the narratives for the presence of situational elicitors of gheirat, building on themes identified in Study 1, and then analyzed the different components of the gheirat experience to detect the differences based on the presence or absence of each elicitor.

\section{Gender Differences in Gheirat Experience}

Given the prominence of gender in conceptions of gheirat, our third goal was to examine whether the intensity of gheirat, and the relative importance of the various experiential and behavioral components, varied between men and women. 


\section{Methodological Approach}

Rationale for a multi-dimensional approach. Emotional experiences can be described on one or more dimensions. Higher dimensionality in describing an emotional experience provides a more nuanced and accurate description of the emotional experience as well as the means to differentiate between diverse emotional experiences. For example, a unidimensional approach that places emotional experiences on the positive-negative continuum would categorize anger as a negatively valanced emotional experience; but might not be effective in distinguishing different types of anger (e.g., self-directed vs. other-directed anger; Becker et al., 2011; Hansen \& Sassenberg, 2011). Adding other dimensions (e.g., situational appraisals, somatic experiences) will generate a richer description that can speak to the similarities and differences among different variants of an emotional experience. Accordingly, given the previously discussed goals of this study to provide an in-depth understanding of participants' experience of gheirat and its variants, we adopted a multi-dimensional approach grounded in theories of emotion and the findings from the qualitative study.

Ensuring cultural sensitivity. To investigate the various dimensions of participants' experience of gheirat, we asked them to complete theoretically relevant measures following their written narratives. Consistent with the theoretical perspective that treat emotional experiences as multi-component processes (discussed earlier), we adopted measures of different components that have either been developed for cross-cultural research or used in multiple cultural settings before. Given our emphasis on an emic-etic approach, we took a number of steps to ensure the cultural sensitivity and applicability of these measures. First, scale and item selection were fully informed by the themes from the previous study and the literature on gheirat. For example, to examine participants' affective experiences during the gheirat episode, we adopted items from 
the Positive and Negative Affect Scale (PANAS-X; Watson \& Clark, 1994) because this scale provided a large repertoire of emotion words that allowed for a granular measurement of affective experiences and has been previously used in this cultural context (e.g., Shokri et al., 2014). Of the sixty emotion words in the original scale, we selected and preregistered 25 specific items (Table OS3) that were deemed relevant based on participants' discussions of gheirat in Study 1 and the prior literature (e.g., Bakhtiar, 2015). A similar selection process was applied to other measures of bodily symptoms, behavioral responses, and cognitive appraisals (adapted from Matsumoto et al. [1988] and Wallbot \& Scherer [1986]). Only selected items from these scales were administered to participants.

Second, as part of the selection and adaptation process, translated scales were reviewed by five cultural informants who provided feedback on the face validity, clarity, and cultural sensitivity of the items. This feedback informed our item adaptation and translation process (see the Online Supplement for more details). Third, when calculating composite scores using adapted scales, the component structures were either decided and preregistered a priori based on the findings from Study 1, or decided in a data-driven manner (using principal component analysis). Finally, for one of the key outcomes (i.e., appraisals) where we realized that the established measures do not comprehensively capture the themes that emerged in the previous study, we developed a gheirat-specific measure based on the gheirat literature and the insights from the interviews.

Our primary aim in this study was to deepen our understanding of gheirat through a descriptive approach (Rozin, 2001; Scheel et al., 2021). We created a preregistration (https://osf.io/3p69m) that identified this as a primarily exploratory study, but which also included analyses for ten specific hypotheses that were conjectures based on background 
knowledge and the qualitative interviews. Because the set of preregistered analyses was not comprehensive, and were not based on a systematic approach, we have chosen to present the results without separating or prioritizing the preregistered analyses. Instead, for clarity of presentation we have organized the results around the research questions listed above. For transparency, we present results for all preregistered analyses in the online supplement. Because of the quasi-exploratory approach, we adjust the alpha threshold and calibrate our interpretations of the results as cautionary steps against over-generalization. Namely, for non-preregistered analyses, we set the threshold for significance at $p<.005$ and report the results of analyses with p-values between .05 and .005 as "suggestive" (see Benjamin et al., 2018).

\section{Method}

\section{Participants}

Considering the novelty of the topic, we aimed for a target sample size of at least 300 participants, based on feasibility of data collection, and the finding that correlations tend to stabilize around this sample size (Schönbrodt, \& Perugini, 2013). Furthermore, because of the nature of the phenomenon and its relation to participants' gender, it was important to have complete responses from at least 150 male participants. As such, using Qualtrics' "Report" function, the completion rates and gender distribution were checked every other day, without running any analyses, until the target sample size was achieved.

We collected data from 394 Iranian adults (50.5\% identified as female; $M_{\text {age }}=31.17$, $\left.S D_{\text {age }}=7.66\right)$ who learned about the study through Telegram (a popular social networking platform in Iran, with a high penetration rate; Jafari, 2017) and participated in exchange for personality feedback. Due to cultural and legal sensitivities, we did not ask participants to report sexual orientation or non-binary gender. Consistent with the preregistration, $32(8 \%)$ responses 
were excluded because the open-ended narratives were missing or non-compliant with the instructions. Responses were coded as non-compliant if they did not provide any details about a specific personal event or experience that led to the experience of gheirat (e.g., "I don't remember exactly. To the extent that I remember, anger, confusion, feeling of not being taken seriously", "I believe this concept [gheirat] is wrong, and it's wrong that we were taught about it"). The remaining sample ( $N=362,52 \%$ female, $47 \%$ male) achieved our preregistered goal of having at least 150 responses from these gender categories. A majority of the participants (92\%) reported residing in Iran, $84 \%$ reported that they have had a romantic partner in the past, and $64 \%$ reported having a romantic partner now. Consistent with recent surveys of the changing pattern of religiosity among Iranians (e.g., Maleki \& Arab, 2020), the participants reported low to moderate levels of religiosity $(M=2.29, S D=1.16$, on a $1-5$ scale). Sensitivity analyses (Faul et al., 2009) indicate that the final sample size $(N=362$ ) has $80 \%$ power (when $\alpha=.05$, twotailed) to detect small-to-moderate effect sizes for correlational analysis $(|\rho|=.15)$, withinsubjects mean differences $\left(d_{\mathrm{z}}=0.15\right)$, gender differences $(d=0.30)$, linear regression models with three predictors $\left(f^{2}=.03\right)$, and within-between interaction effects in a 2 X 2 mixed ANOVA $(f=0.07)$.

\section{Procedure and Materials}

After providing consent, participants were instructed to recall an instance when they experienced gheirat and write about this episode, providing details about the situation and their reaction. They were then asked to respond to items about the recalled episode. The study was administered in Persian/Farsi. The established measures that were developed in English were translated using the back-translation method (Brislin, 1970). All study materials, data, analyses code and output reproducing the results are available at https://osf.io/pxsbv/ 
Affective experience. We measured affective experiences related to the experience of gheirat using 25 items from the Positive and Negative Affect Scale (PANAS-X; Watson \& Clark, 1994). Items were rated on a 5-point Likert scale (not at all to a lot).

Somatic symptoms. Using a list of ten bodily symptoms (e.g., lump in throat, heart beating faster), participants were instructed to report all symptoms that they experienced during the gheirat episode using one of three responses for each symptom (yes/no/don't remember). These items were taken from Wallbot and Scherer (1986), and Matsumoto et al. (1988).

Expressive and behavioral reactions. Using a list of 10 items, participants reported their behavioral, verbal, and expressive reactions during the experience of gheirat (response options: yes/no/don't remember). Additionally, participants reported how much they tried to hide their feelings from others during the episode $(1=$ not at all, $3=$ very much $)$. These items were also taken from Wallbot and Scherer (1986), and Matsumoto et al. (1988).

General appraisals. Participants evaluated eight situational aspects of the episode (items adapted from Wallbott and Scherer, 1986). Appraisal items measured expectedness, unfairness, and appearance of impropriety (responses for all three items range from $1=$ not at all to $3=$ very $m u c h)^{2}$, pleasantness (response options: pleasant, neutral, unpleasant), influence on goal pursuit (response options: it helped, it was neutral, it hindered), responsibility (i.e., "who do you think was responsible for the event in the first place?") with eight mutually exclusive response options (e.g., "you," and "fate/destiny"), effects on self-esteem, and effects on their relationship with the

\footnotetext{
${ }^{2}$ We originally planned to use different response options for the unfairness item (fair, neutral, unfair) and the impropriety item (improper, neutral, proper). However, the options were mistakenly entered in Qualtrics as 1= "not at all" to $3=$ "very much." While deviating from the original plan, these response options still allow for the test of the preregistered hypotheses that a gheirat eliciting event is generally perceived as "unfair" and "improper/immoral."
} 
target (response options: negative, no effect, positive). In addition to the response options listed above, all items included an "I don't know" choice.

Gheirat-specific appraisals. Based on insights from the Study 1 interviews, we developed 20 items reflecting appraisals of the gheirat-eliciting situation (e.g., "I felt/sensed that someone is being victimized"). Participants rated these items on a 5-point scale (not at all to extremely).

Gheirat intensity and other characteristics of the episode. Participants were asked to report the intensity of their experience of gheirat during the episode on a 5-point scale $(1=$ not at all, $5=$ extremely; $M=3.73, M d n=4, S D=0.97)$, as well as other characteristics of the episode (e.g., the target of gheirat). These characteristics are reported in the online supplement.

\section{Mixed-Method Coding of Situational Elicitors}

One of the goals of this study was to explore the similarities and differences in the experience of gheirat due to different situational elicitors. To do this, the first and second authors coded the narratives for situational elicitors. We used the three themes identified in Study 1 (i.e., the three types of concerns that led to gheirat) as the starting point; but in keeping with the exploratory approach of this study, the code definitions were refined in an iterative process. First, each coder analyzed 10 narratives independently. Next, the two coders shared the results of their analysis and discussed potential categories or code definitions that can better describe the situational elicitors in the narratives. After repeating this process for 50 narratives, it became clear that each gheirat-eliciting situation involved one or two of the following themes: (a) perception of harm or insult to namoos, (b) a third person violating namoos' boundary, or (c) namoos violating a norm or boundary. Afterwards, all narratives were coded (independently of the rest of the dataset) by the two coders (i.e., the first and second authors) for the presence or 
absence of each category $(0=$ absent, $1=$ present $)$. We did not consider the elicitors to be mutually exclusive, so we coded the presence or absence of each elicitor separately to allow multiple elicitors in a single narrative. After all narratives were coded, inter-rater reliability analysis indicated substantial agreement for all categories $\left(\kappa_{\mathrm{a}}=.86, \kappa_{\mathrm{b}}=.78, \kappa_{\mathrm{c}}=.84\right)$. Where there was a disagreement in ratings, it was resolved by discussion between the coders.

Based on the final agreed-upon codes, the difference in the frequency of themes was significant (Cochran's $Q(2)=26.53, p<.001)$, with the most common theme being boundary violation by a third person (55\%) followed by perception of harm or insult to namoos (43\%) and boundary violation by namoos $(33 \%)$. The gheirat-elicitors in $68 \%$ of the narratives were consistent with only one theme, while the rest were consistent with two themes.

\section{Results}

\section{A Prototypical Gheirat Episode}

Affective experience. To investigate the affective dimensions of a prototypical gheirat episode, we conducted the following series of non-preregistered analyses. First, as an initial variable-reduction step, we used a principal components analysis (PCA) with varimax rotation on the participants' ratings of 25 emotions (see online supplement; this was not pre-registered). We used PCA as a data-driven strategy to reduce the dimensionality of the variables collected from this sample. The goal was not to indicate latent variables, which require other analytical approaches such as exploratory factor analysis. The PCA resulted in a four-component solution: (a) Hostility, consisting of seven emotions (angry, loathing-hateful, hostile, disgusted, upset, excited, and irritable), (b) Self-blame, consisting of four emotions (angry at self, guilty, disgusted with self, embarrassed), (c) Bold/empowered, consisting of seven emotions (lively, proud, enthusiastic, happy, daring, strong, and relaxed), and (d) Socially fearful, consisting of four 
emotions (afraid, shy, anxious, lonely). The four components cumulatively explained $60 \%$ of the total variance and had eigenvalues between 5.43 and 1.29 (see Figure OS2 and Tables OS3 and OS4 for detailed results). We calculated scale scores for each emotion component by averaging the corresponding items. See Table 1 for correlations among the scales, internal consistencies, and means.

Table 1.

Internal Consistencies, and Intercorrelations Among the Four Emotion Categories

\begin{tabular}{lccccc} 
& Hostility & $\begin{array}{c}\text { Self- } \\
\text { blame }\end{array}$ & $\begin{array}{c}\text { Bold/ } \\
\text { Empowered }\end{array}$ & Socially Fearful & $M(S D)$ \\
\hline Hostility & $(.87)$ & & & & $3.35(0.92)$ \\
Self-blame & .26 & $(.76)$ & & & $1.97(0.86)$ \\
Bold/empowered & $-.02^{\dagger}$ & $-.15^{*}$ & $(.88)$ & & $1.98(0.91)$ \\
Socially fearful & .40 & .51 & -.18 & $(.73)$ & $2.42(0.89)$ \\
\hline
\end{tabular}

Note. Internal consistencies (Cronbach's $\alpha$ s) are displayed in the diagonal (within parentheses). ${ }^{\dagger} p=.729 ; * p=.004$; For the rest of the correlations, $p \mathrm{~s}<.001$.

Second, to investigate the within-subjects differences in the emotions associated with the experience of gheirat, a non-preregistered one-way ANOVA with emotion category as the independent variable was conducted, which revealed a significant difference in the intensity of different emotion categories during a gheirat episode, $F(3,1071)=219.17, p<.001, \eta^{2}{ }^{2}=.38$. Post-hoc analyses showed that the intensity of the hostile emotion category was significantly higher than self-blame $\left(d_{z}=1.29^{3}\right)$, bold/empowered $\left(d_{z}=1.07\right)$, and socially fearful category $\left(d_{z}\right.$ $=0.94)$. Furthermore, the intensity of socially fearful emotion category was higher than selfblame $\left(d_{z}=0.53\right)$ and bold/empowered emotions $\left(d_{z}=0.39 ;\right.$ all $\left.p \mathrm{~s}<.001\right)$. There was no

\footnotetext{
${ }^{3}$ The effect size calculations for paired-samples t-tests are based on Cohen's $d_{z}$ formula which accounts for the correlation between variables (see Cohen, 1988, and Lakens, 2013).
} 
significant difference between the self-blame and bold/empowered emotions, $p=.801, d_{z}=0.01$ (Table 1).

Reported somatic symptoms. Endorsement of different physiological experiences is presented in Figure 1. We tested a preregistered hypothesis that four somatic symptoms associated with high arousal (i.e., "Change in breathing, feeling hot, cheeks burning, heart beating faster, muscles tensing, trembling") were more common than two symptoms associated with low arousal (i.e., "Feeling warm pleasant, muscles relaxing, restful.") To test this, consistent with the preregistration, a high arousal and low arousal score was calculated for each participant by dividing the number of symptoms reported as experienced by the total number of symptoms in that category, and then compared using a paired-samples t-test. Consistent with expectations, high-arousal symptoms were much more common than low-arousal symptoms when experiencing gheirat, $t(348)=26.19, p<.001, d_{z}=1.40$.

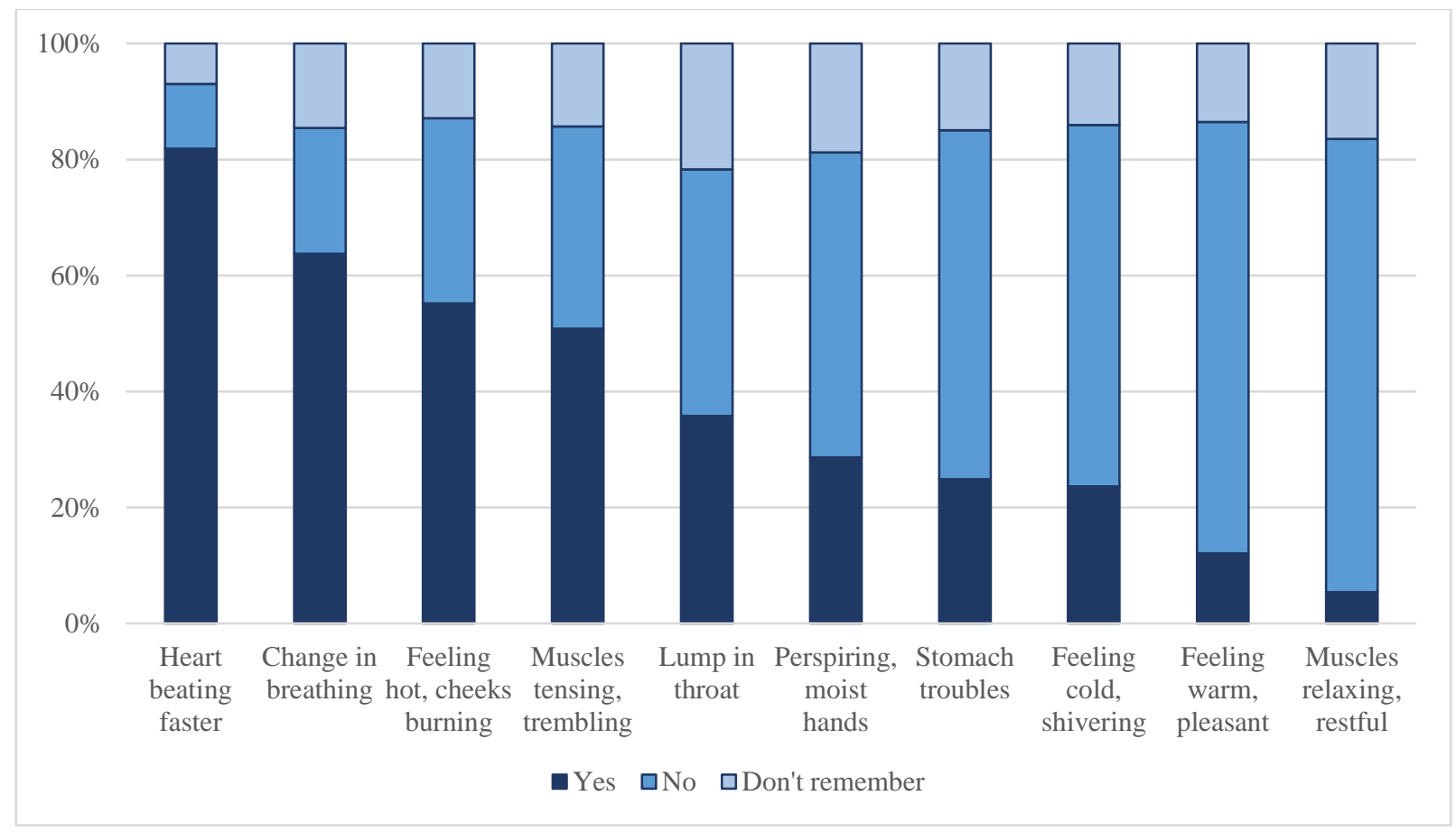

Figure 1. Participants' reported somatic symptoms during the experience of gheirat. 
Reported expressive and behavioral reactions. Endorsement of different expressive reactions is presented in Figure 2. We tested the preregistered hypothesis that negative expressive reactions (i.e., "Screaming, yelling", "moving away/withdrawing from people," “aggression") will be experienced more than positive reactions (i.e., "laughing, smiling”). Similar to the somatic symptoms, proportion scores for these expressive reactions were calculated for each participant. The results of within-subjects comparisons showed that, as predicted, participants had more negative expressive reactions when experiencing gheirat, $t(347)$ $=12.23, p<.001, d_{z}=0.66$.

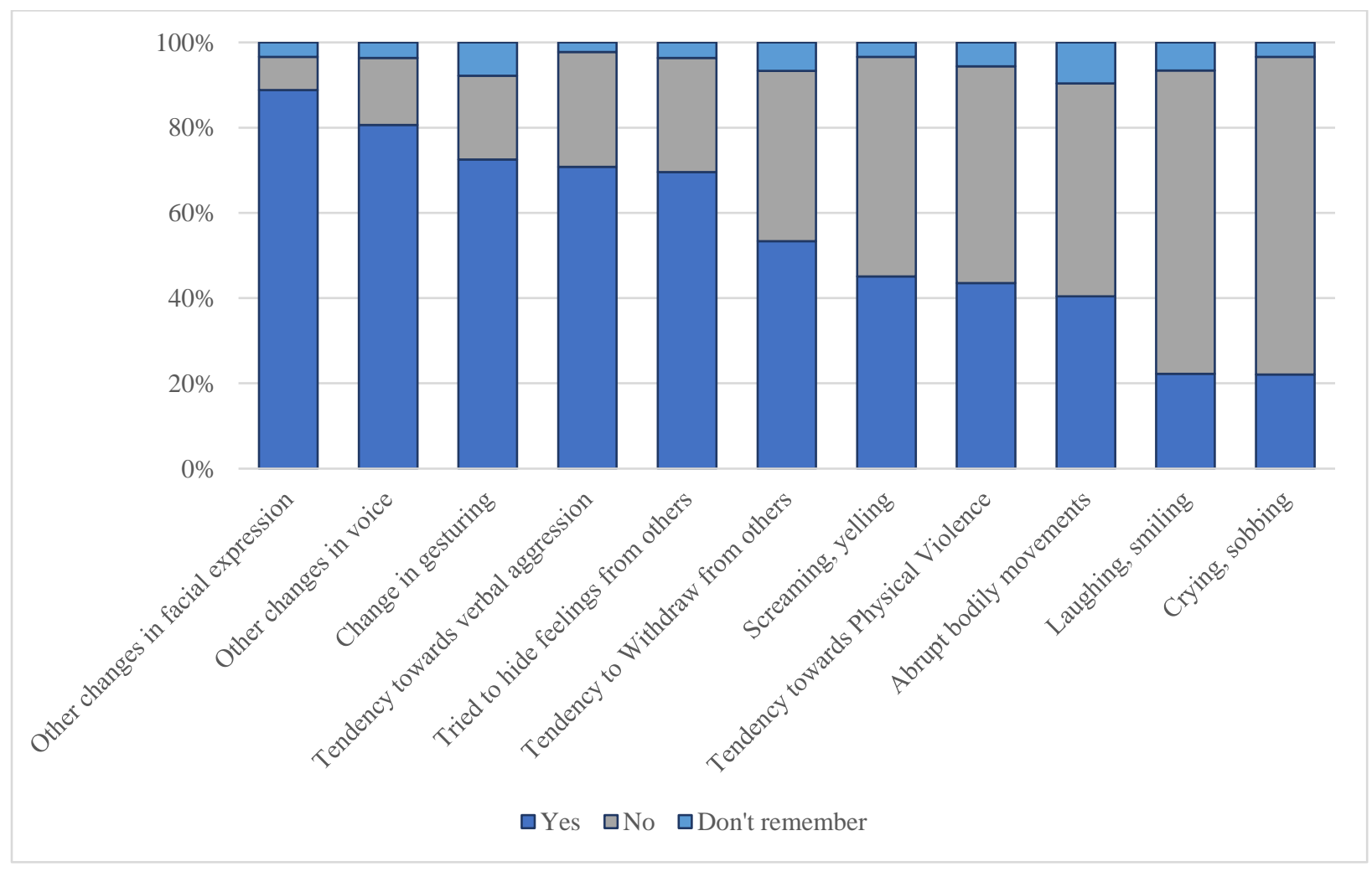

Figure 2. Participants' reported behavioral/expressive responses during the experience of gheirat. Note. Other changes in facial expression refers to expressions other than "laughing/smiling" and "crying/sobbing." Other changes in voice refers to changes other than "screaming/yelling." 
General appraisals. We tested a preregistered hypothesis that the experience of gheirat, and the situations eliciting it, are more likely to be categorically evaluated as unpleasant, unfair, and improper or immoral. Three chi-square tests supported these predictions. Eighty-two percent of participants perceived the experience of gheirat as unpleasant $\left(\chi^{2}(2)=362.34, p<.001\right), 83 \%$ rated the situation eliciting gheirat as somewhat or very unfair $\left(\chi^{2}(1)=135.12, p<.001\right)$, and $65 \%$ rated the behavior eliciting gheirat would be evaluated as somewhat or very improper and immoral by an acquaintance $\left(\chi^{2}(1)=28.83, p<.001\right)$. For further analyses of other appraisals, see Table OS2.

Gheirat-specific appraisals. To investigate the gheirat-related appraisals, we conducted the following series of non-preregistered analyses. We ran a PCA on the 20 items measuring gheirat-specific appraisals to summarize these variables into fewer dimensions (see Online Supplement for details). A three-component model emerged: (a) Insecurity (seven items; e.g., I felt "hopeless," "belittled," "my reputation is at risk"); (b) Responsibility: (five items; e.g., I felt "responsible for someone," "protective towards someone," "someone is being oppressed"); (c) Self-worth: (five items; e.g., I felt "respected," "liked," "focused."). The three components had eigenvalues ranging from 4.70 to 1.71 (see Figure OS5) and cumulatively explained $57 \%$ of the total variance (see Tables OS6 and OS7). Based on these results, we calculated scale scores for each appraisal component by averaging the responses to the corresponding items. Intercorrelations and internal consistencies are reported in Table 2.

A non-preregistered repeated-measures one-way ANOVA revealed significant differences in the intensity of different appraisal components during a gheirat episode, $F(2,714)$ $=329.64, p<.001, \eta_{p}^{2}=.48$. Post-hoc analyses revealed that the intensity of responsibility 
appraisals experienced by participants was significantly higher than insecurity $\left(d_{z}=0.69\right)$ and self-worth appraisals $\left(d_{z}=1.62\right)$, and insecurity appraisals were significantly higher than selfworth $\left(d_{z}=0.74 ; p \mathrm{~s}<.001\right)$.

Table 2.

Intercorrelation between Appraisals, and Correlations with Emotion Categories

\begin{tabular}{llcccc} 
& & Insecurity & Responsibility & Self-worth & M $(S D)$ \\
\hline Appraisals & Insecurity & $(.87)$ & & & $2.41(1.04)$ \\
& Responsibility & $.11^{\dagger}$ & $(.80)$ & & $3.37(1.04)$ \\
& Self-worth & $-.27^{*}$ & $.27^{*}$ & $(.73)$ & $1.61(0.70)$ \\
Emotional experiences & Hostility & $.47^{*}$ & $.46^{*}$ & -.01 & $3.35(0.92)$ \\
& Self-blame & $.49^{*}$ & .08 & $-.20^{*}$ & $1.97(0.86)$ \\
& Bold/empowered & $-.27^{*}$ & $.28^{*}$ & $.70^{*}$ & $1.98(0.91)$ \\
& Socially fearful & $.66^{*}$ & .07 & $-.21^{*}$ & $2.42(0.89)$ \\
\hline
\end{tabular}

Note. Internal consistencies (Cronbach's $\alpha$ ) are displayed in the diagonal (within parentheses). ${ }^{\dagger} p=.044 ; * p<.001$.

\section{Situational Elicitors and Variations in Experience}

Variations in affective experience. To investigate how the participants' emotional experiences differed in response to different elicitors, we ran a non-preregistered regression model for each emotion category. In each model, the intensity of the emotion category was the outcome, and the presence of each of the three types of elicitors were the predictors (dummy coded: 0 = absent, 1 = present; all three codes were entered in the same model; Figure 3). There was no difference in the intensity of hostility in relation to the different elicitors, $F(3,345)=$ $0.77, p=.515, R^{2}=.01$. However, the intensity of the other emotion categories varied as a result of different elicitors $\left(p \mathrm{~s} \leq .008, .03 \leq R^{2} \leq .15\right)$. Specifically, participants reported significantly higher intensity of self-blame when violation by namoos was present (vs. absent), $B=.38, \beta=$ $.21, t(345)=3.03, p=.003$. Furthermore, participants had a significantly higher intensity of 
bold/empowered emotions when the situation created a perception of harm or insult, $B=.46, \beta=$ $.25, t(345)=3.28, p=.001$, but they had a significantly lower level of bold/empowered emotions when there was a violation by namoos, $B=-0.36, \beta=-.19, t(345)=-2.85, p=.005$. Finally, when there was a violation by namoos, participants experienced much higher levels of socially fearful emotions, $B=0.64, \beta=.34, t(345)=4.96, p<.001$. Additional non-preregistered models indicated that controlling for gender did not substantially change any of the effects. It did, however, reveal a main effect where men reported higher levels of self-blame emotions, $B=$ $0.28, \beta=.16, t(341)=2.93, p=.004$.

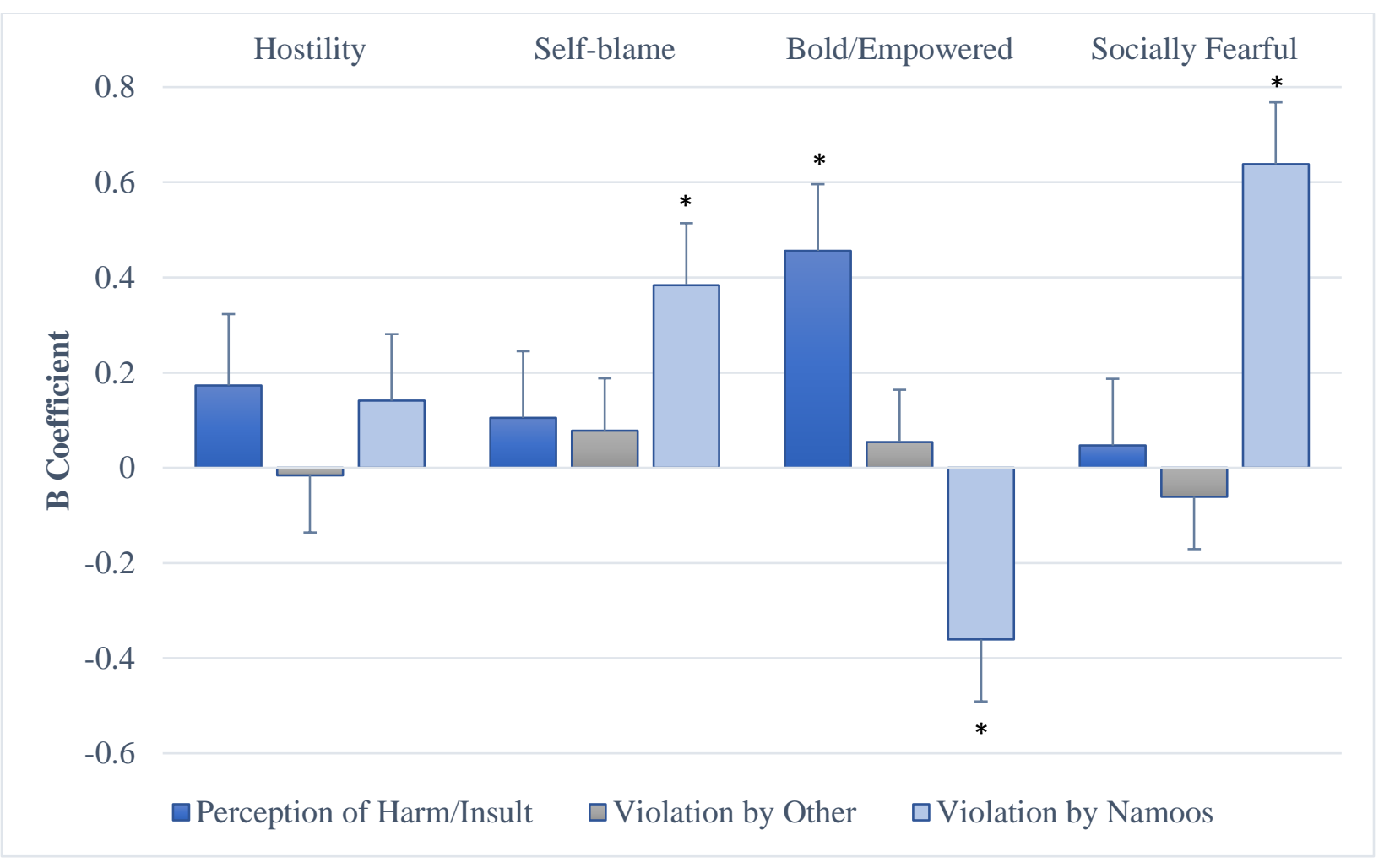

Figure 3. Affective experiences during the gheirat episode as a function of presence (vs. absence) of different violations. Y-axis represents unstandardized $B$ coefficients, and the error bars are SEs. * indicates $p<.005$.

Variations in somatic symptoms. Non-preregistered chi-squared tests indicated that the ratings of somatic symptoms were largely independent from the three types of gheirat elicitors, 
with two exceptions. Stomach trouble was more common in the presence of a boundary violation by namoos $\left(42.5 \%\right.$ vs. $\left.22.3 \% ; \chi^{2}(1)=12.45, p<.001\right)$. Results were suggestive of a similar pattern for feeling cold and shivering (37.7\% vs. $\left.22.7 \% ; \chi^{2}(1)=6.16, p=.013\right)$.

Variations in expressive and behavioral reactions. A series of non-preregistered chisquare tests revealed that gheirat-eliciting situations led to different expressive responses (Figure 4). In response to perception of harm to namoos, aggressive reactions (e.g., screaming/yelling) were more common. Furthermore, participants had a lower tendency to hide their feelings or, suggestively, withdraw from others. In contrast, in response to violation by namoos, more participants reported a tendency to withdraw from others, or hide their feeling or mask it with other expressions (e.g., sadness); results were also suggestive of a lower tendency towards aggression. Presence or absence of violation by other did not lead to significantly different expressive responses. 


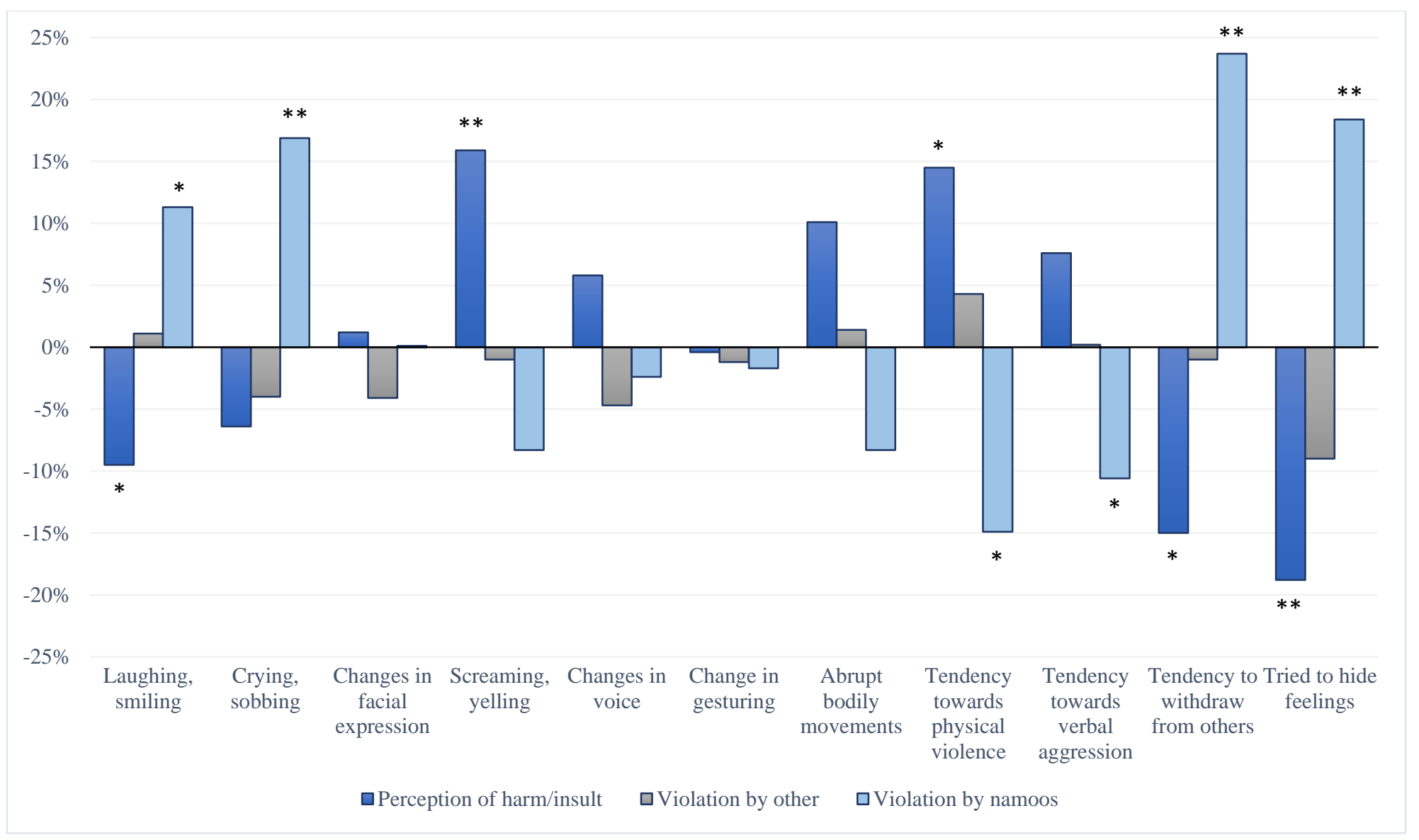

Figure 4. Expressive responses as a function of different gheirat elicitors.

Note. Values on the $y$-axis are the difference between the percentage of participants who reported the expressive response when the corresponding elicitor was present vs. absent. Detailed report of the analyses is provided in the Online Supplement.

$* .05>p \geq .005$ (i.e., suggestive); $* * p<.005$ (i.e., significant). 
Variations in gheirat-specific appraisals. We conducted a non-preregistered regression model for each appraisal category. In each model, the appraisal category was the outcome and the presence of each of the three types of gheirat-elicitors were the predictors (dummy coded: $0=$ absent, 1 = present; all three codes were entered in the same model; Figure 5). The first model (outcome: insecurity) indicated that insecure appraisals were considerably higher when there was a violation by namoos, $B=.76, \beta=.34, t(345)=5.19, p<.001$. The next model (outcome: responsibility) demonstrated that perception of harm was strongly associated with higher intensity of responsibility appraisals, $B=0.73, \beta=.35, t(345)=4.88, p<.001$, whereas violation by namoos was significantly associated with lower responsibility appraisals, $B=-0.47, \beta=-.21$, $t(345)=-3.39, p<.001$. The third model (outcome: self-worth) demonstrated that violation by namoos was associated with significantly lower levels of self-worth, $B=-0.30, \beta=-.21, t(345)=$ $-3.00, p=.003$

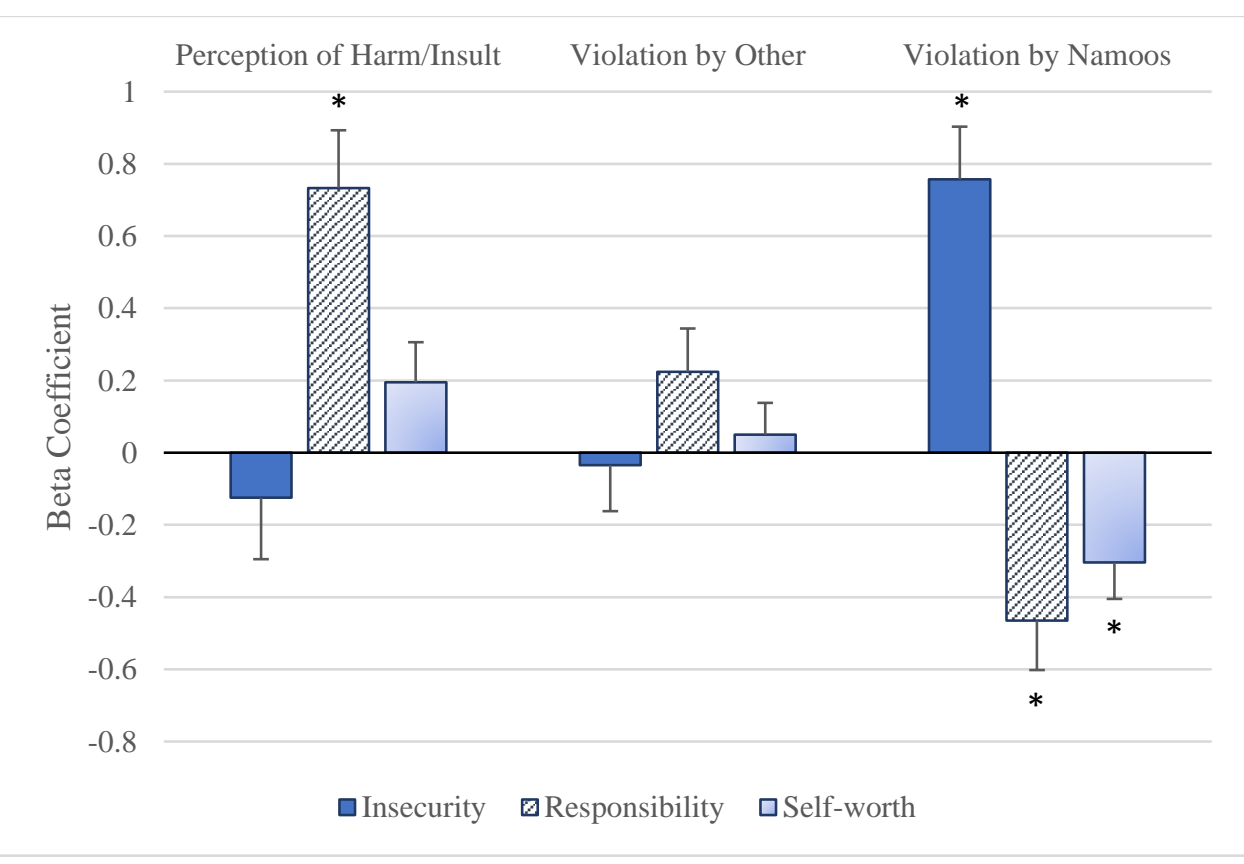

Figure 5. The association between the three types of elicitors and the three appraisal categories. Note. Y-axis represents unstandardized $B$ coefficients, and the error bars are SEs. ${ }^{*} p<.005$. 


\section{Gender Differences}

Frequency of the gheirat-elicitors. Exploratory analyses indicated that a larger proportion of men than women wrote scenarios where the elicitor included a perception of harm or insult to namoos $\left(51 \%\right.$ vs. $\left.37 \% ; \chi^{2}(1)=7.50, p=.006\right)$ or boundary violation by a third person $\left(66 \%\right.$ vs. $\left.48 \% ; \chi^{2}(1)=11.24, p<.001\right)$. In contrast, a higher proportion of women wrote scenarios where gheirat was elicited due to boundary violation by namoos $\left(44 \%\right.$ vs. $22 \% ; \chi^{2}(1)=$ $17.51, p<.001)$.

Intensity and frequency of gheirat. Consistent with the folk theories of gheirat as a primarily male experience, we tested the preregistered hypothesis that men will report a higher intensity of gheirat compared to women. Participants' intensity of gheirat during the episode was measured using a single item $(M=3.73, S D=.097)$. Contrary to our preregistered prediction, the results revealed a slightly higher intensity of gheirat reported by female participants, $t(350)=$ $2.12, p=.035, d=0.23$. Furthermore, we tested the preregistered hypothesis that men, in general, will report a higher frequency of gheirat compared to women. To test this, consistent with the preregistration, we calculated the mean gheirat frequency across 11 targets for each participant $(\alpha=.89)$ and compared the mean frequency of male and female participants. There was not a significant gender difference, $t(342)=0.72, p=.470, d=0.08$. Overall, these results are contradictory to the common notion that gheirat is a primarily male experience.

Gender differences in experience. Overall, non-preregistered analyses of gender differences in the various experience domains revealed a few interesting differences and many similarities:

Analyses of the 4 emotion components showed no significant gender differences. 
In analyses of the somatic symptoms, significantly more women reported feeling cold, shivering $\left(35.5 \%\right.$ vs. $\left.18.1 \% ; \chi^{2}(1)=10.59, p=.001\right)$. Additionally, results were suggestive that more men reported heart beating faster $\left(93.0 \%\right.$ vs. $\left.83.3 \% ; \chi^{2}(1)=6.37, p=.012\right)$. There were no significant gender differences in other physiological symptoms $\left(\chi^{2} \mathrm{~s} \leq 3.13, p \geq .077\right)$.

In the expressive and behavioral reports, a significantly larger percentage of women reported laughing, smiling $\left(33 \%\right.$ vs. $\left.14 \% ; \chi^{2}(1)=15.14, p<.001\right)$ and crying, sobbing (34\% vs. $\left.10 \% ; \chi^{2}(1)=26.59, p<.001\right)$. In contrast, a significantly larger percentage of men reported a tendency towards physical violence in response to elicitation of gheirat $\left(55 \%\right.$ vs. $38 \% ; \chi^{2}(1)=$ $10.51, p=.001)$. There were no gender differences in the other expressive responses $\left(\chi^{2} \mathrm{~s} \leq 2.84\right.$, $p s \geq .092)$. See Figure OS3 for an overview of the gender differences.

In the general appraisal items, the results (Table OS5) were suggestive that men were more likely to evaluate the event that led to gheirat as improper and immoral, and women were more likely to consider the event as a negative influence on their relationship with namoos.

In the gheirat-specific appraisal items, responsibility appraisals were higher among men $(t(353.5)=4.34, p<.001, d=0.46)$. Results were also suggestive that Insecurity appraisals were higher among women $(t(352.1)=2.18, p=.030, d=0.23)$, and Self-worth appraisals were higher among men $(t(354.7)=2.29, p=.023, d=0.24)$.

\section{Discussion}

Broadly speaking, in the face of a gheirat-eliciting situation, people typically experience a sense of responsibility towards others, insecurity about the self, and low self-worth. These appraisals are joined with the affective experiences of hostility (e.g., anger, disgust) and social fear (shyness, loneliness), and physiological symptoms often associated with high arousal (e.g., heart beating faster). Behavioral responses generally consist of approach-oriented expressions 
and aggressive tendencies but could also include a tendency to withdraw from others. The gheirat episode is generally perceived as unpleasant, with negative influence on one's selfesteem and relationship with namoos. Furthermore, the behavior that triggers the experience of gheirat is considered to be unfair and improper or immoral. These results shed light on the features and characteristics of the prototype of gheirat. However, there is considerable variability around this typical experience depending on the situation that elicits gheirat.

Analysis of the participants' narratives was consistent with the findings from the first study indicating that the presence or absence of three types of elicitors leads to three "variants" of gheirat, each of them having distinct affective and cognitive features (Table 3). When there is a violation by other (usually of a sexual or romantic nature), the features of the experience of gheirat are the closest to the gheirat prototype. When there is a perception of harm or insult to namoos, the experience of gheirat includes a stronger sense of protectiveness and responsibility. In addition to the negative affective experiences associated with the prototype of gheirat (e.g., hostility), this variant is associated with a cluster of positive emotions reflecting a feeling of boldness and empowerment (e.g., proud, daring). Even though this experience is perceived as unpleasant, it also leads to an increase in one's self-esteem and improvement of one's relationship with namoos. All together, these results suggest that a perception of harm or insult to namoos triggers a cluster of affective and cognitive reactions aimed at changing and influencing the social environment in a way that the possibility of future harm or insult is removed.

When there is a perception of violation by namoos, the experience of gheirat is quite different. It includes a stronger sense of insecurity and lower self-worth. Compared to the gheirat prototype, this variant is associated with more self-blaming (e.g., angry at self) and socially fearful (e.g., lonely and anxious) emotions. The behavioral and expressive tendencies often 
revolve around withdrawing from others and sometimes hiding or masking one's emotions.

Furthermore, the overall experience is perceived as harmful to one's self-esteem and to the relationship with namoos. In contrast to gheirat in response to perception of harm, the affective and cognitive reactions to violations by namoos are indicative of an avoidant orientation and a tendency to withdraw from others following the feeling of insecurity and diminished self-worth. For a summary of the findings, see Table 3 . 
Table 3.

Features of the Prototype of Gheirat and its Variants

\begin{tabular}{|c|c|c|c|c|c|}
\hline & & & Variants & & \\
\hline & Prototype & Perception of harm & Violation by other & Violation by namoos & Gender difference \\
\hline Affect & $\begin{array}{l}\text { Hostile }>\text { Socially fearful } \\
>\text { Bold }=\text { Self-blaming }\end{array}$ & More Bold & No difference & $\begin{array}{l}\text { More Self-blaming and } \\
\text { Socially fearful, less } \\
\text { Bold }\end{array}$ & No difference \\
\hline Appraisals & $\begin{array}{l}\text { Responsibility }> \\
\text { Insecurity }>\text { Self-worth }\end{array}$ & $\begin{array}{l}\text { Higher } \\
\text { Responsibility }\end{array}$ & No difference & $\begin{array}{l}\text { Higher Insecurity, lower } \\
\text { Responsibility, lower } \\
\text { Self-worth }\end{array}$ & $\begin{array}{l}\text { Men: higher } \\
\text { Responsibility; } \\
\text { Women: higher } \\
\text { Insecurity }\end{array}$ \\
\hline $\begin{array}{l}\text { Physiological } \\
\text { symptom }\end{array}$ & $\begin{array}{l}\text { Strong indicators of high } \\
\text { arousal (e.g., "heart } \\
\text { beating faster," "change in } \\
\text { breathing," "feeling hot, } \\
\text { cheeks burning") moderate } \\
\text { indicators of anxiety (e.g., } \\
\text { "feeling cold, shivering") }\end{array}$ & Similar to prototype & No difference & $\begin{array}{l}\text { More "stomach } \\
\text { trouble"; more "feeling } \\
\text { cold, shivering"" }\end{array}$ & $\begin{array}{l}\text { Women: more } \\
\text { "feeling cold, } \\
\text { shivering"; Men: more } \\
\text { "heart beating faster" }\end{array}$ \\
\hline $\begin{array}{l}\text { Expressive/behavioral } \\
\text { response }\end{array}$ & $\begin{array}{l}\text { Most common: Changes in } \\
\text { face, voice, and gesture, a } \\
\text { tendency towards verbal } \\
\text { aggression or hiding } \\
\text { feelings. }\end{array}$ & $\begin{array}{l}\text { More } \\
\text { "screaming/yelling,"; } \\
\text { "tendency towards } \\
\text { physical violence"; } \\
\text { less attempt to hide } \\
\text { feeling, lower } \\
\text { tendency to } \\
\text { withdraw }\end{array}$ & No difference & $\begin{array}{l}\text { Stronger "tendency to } \\
\text { withdraw from others;" } \\
\text { more "attempting to } \\
\text { hide the feelings," } \\
\text { "crying/sobbing," and } \\
\text { "laughing/smiling"; less } \\
\text { tendency towards verbal } \\
\text { and physical violence }\end{array}$ & $\begin{array}{l}\text { Women: more } \\
\text { "smiling/laughing," } \\
\text { "crying/sobbing"; } \\
\text { Men: stronger } \\
\text { "tendency toward } \\
\text { physical violence" }\end{array}$ \\
\hline Pleasantness & $\begin{array}{l}\text { Overwhelmingly } \\
\text { unpleasant }\end{array}$ & More unpleasant & More neutral & Similar to prototype & No difference \\
\hline Fairness & Overwhelmingly unfair & More unfair & Less unfair & Similar to prototype & No difference \\
\hline
\end{tabular}




\begin{tabular}{|c|c|c|c|c|c|}
\hline $\begin{array}{l}\text { Appearance of } \\
\text { impropriety }\end{array}$ & $\begin{array}{l}\text { Generally } \\
\text { improper/immoral }\end{array}$ & Similar to prototype & No difference & Similar to prototype & $\begin{array}{l}\text { More men: Very } \\
\text { improper/immoral }\end{array}$ \\
\hline $\begin{array}{l}\text { Influence on goal } \\
\text { pursuit }\end{array}$ & Generally no influence & $\begin{array}{l}\text { More: helped goal } \\
\text { pursuit }\end{array}$ & No difference & $\begin{array}{l}\text { More: hindered goal } \\
\text { pursuit }\end{array}$ & No difference \\
\hline $\begin{array}{l}\text { Influence on self- } \\
\text { esteem }\end{array}$ & More negative & More positive & No difference & More negative & No difference \\
\hline $\begin{array}{l}\text { Influence on } \\
\text { relationship with } \\
\text { namoos }\end{array}$ & More negative or neutral & $\begin{array}{l}\text { More positive or } \\
\text { neutral }\end{array}$ & No difference & More negative & $\begin{array}{l}\text { Women: more } \\
\text { negative; Men: more } \\
\text { positive or neutral }\end{array}$ \\
\hline Who is responsible? & $\begin{array}{l}\text { Most common: Namoos, a } \\
\text { third person, Iran's } \\
\text { cultural environment }\end{array}$ & $\begin{array}{l}\text { More: a third person, } \\
\text { Iran's cultural } \\
\text { environment }\end{array}$ & No difference & $\begin{array}{l}\text { More: Namoos. Less: a } \\
\text { third person, Iran's } \\
\text { cultural environment }\end{array}$ & $\begin{array}{l}\text { Women: more } \\
\text { namoos, men: more } \\
\text { Iran's cultural } \\
\text { environment }\end{array}$ \\
\hline
\end{tabular}

Note. The descriptions under the "Variants" title indicate the difference between the presence vs. absence of the situational elicitor.

The effects in italics are based on suggestive results $(.05>p \geq .005)$; the rest are based on significant results $(p<.005)$. 


\section{Study 3}

In the previous study, participants freely generated the gheirat episode they recalled. A strength of this design is that it provides a naturalistic look into episodes that were experienced by each person and allows an extensive investigation of a prototypical experience of gheirat. However, a limitation is that it is more difficult to make comparisons between features of the eliciting situations or between different people responding to the same situations. For that reason, we complemented the prototype approach by generating a standard set of scenarios to present to participants and then asking them to rate the intensity of gheirat they would feel. We designed these scenarios based on the gheirat literature (e.g., Bakhtiar, 2015) and the themes extracted from Study 1. The scenarios included a wide range of potential targets of gheirat (i.e., romantic partner, female and male relatives, and entities such as country, religion, and career). This provided an opportunity to answer questions about the targets for which gheirat is most commonly experienced. We could also compare how strongly different people responded to the scenarios as a function of the participants' gender, religiosity, traditionalism, and personality.

Following the findings from the qualitative study, we wrote scenarios to have one or more of the following properties: (a) namoos is perceived to be harmed or insulted, (b) a boundary is being violated (by a third person), or (c) there is a violation by namoos (and a perception of loss of namoos to a potential romantic competitor). To provide benchmarks for comparison and validate this approach, we also included "neutral" scenarios in which the three previously mentioned elements are not present (e.g., "if my partner [female] stares at another woman.") $)^{4}$ These scenarios were designed to be parallel to the gheirat-eliciting scenarios in all

\footnotetext{
${ }^{4}$ Note that the term "neutral" is used here to refer to scenarios that, on average, are not expected to elicit gheirat among heterosexual participants. To our knowledge, there is no literature on gheirat among sexual minorities. Furthermore, due to cultural and legal sensitivities surrounding such questions, we did not collect data relevant to
} 
aspects but lacking the distinct element that leads to one of the three hypothesized situational elicitors of gheirat. Consequently, we tested the hypothesis that the neutral scenarios, on average, will elicit less gheirat than the scenarios that have one of the three gheirat-eliciting themes.

Perceiver and target differences. Each participant completed the gheirat scenarios for three different targets: partner, female relative (sister or mother), and male relative (brother or father). This allowed us to test hypotheses about the targets that elicit the strongest gheirat, as well as the gender differences in the intensity of gheirat. According to folk theories, gheirat is perceived as a primarily male experience (Khezr-Heidari, 2018). Furthermore, the most common narratives are about men experiencing gheirat towards their partner or their close female relatives (e.g., sister and mother; Motahhari, 1968). However, folk theories of psychological processes are not necessarily an accurate reflection of the reality (e.g., Dorrough \& Glöckner, 2016;

Terracciano et al., 2005). In the case of gheirat, for example, the results from Study 1 indicated that even though most participants considered gheirat to be generally experienced by men, female participants were also able to describe their own gheirat experiences, some of which were similar to their male counterparts. As such, a systematic test of the hypotheses about the intensity of gheirat as a function of perceiver and target's gender is needed. Specifically, we tested the following hypotheses derived from folk theories of gheirat: (a) Men are more likely than women to report experiencing gheirat towards their partner. (b) Men experience gheirat towards their partner, more than other targets (e.g., their sister, etc.). (c) Men experience gheirat towards female targets in general (partner, sister, mother) more than male targets (brother, father).

Gheirat towards non-relative targets. Namoos extends beyond relatives and consists of entities such as one's country or religion. Examples from discussions of gheirat in the public

sexual orientation during the interviews or for the present study. As such, we are not able to verify that these are appropriate neutral scenarios for individuals who do not identify as heterosexual. 
domain (e.g., political discourse) indicate that gheirat towards non-relative targets can be elicited when the target of an insult or harm is related to one's self and identity (e.g., one's religion or nationality) and the perpetrator is not part of that identity (e.g., a person from a different religion or nationality). For example, Behravesh (2015) point out that as the Iranian nuclear program became a matter of national pride, the political narrative surrounding the issue started to refer to the nuclear program and the scientists involved as namoos, and any potential interference from foreigners as boundary violations (p. 3):

We said we will not allow strangers to conduct any sort of inspection of (our) military centers; [...] We will not allow the smallest insult to the harim [sacred boundary] of our nuclear scientists and scientists in any (other) important and sensitive subject. (Iran's Supreme Leader Ayatollah Khamenei, May, 2015).

In the present study, we tested the hypothesis that the intensity of gheirat will be higher when the perceived "perpetrator" is an outgroup, or when the target of the violation is part of the participants' self or identity (e.g., the participant's religion vs. another religion).

Taking into consideration the folk theory that gheirat is generally stronger when the target of the violation is female, we also tested the hypotheses that, even for the non-relative targets, the intensity of gheirat is higher when the target is female (compared to male). Additionally, considering the strong emphasis on respect for the elderly among Middle Eastern cultures (e.g., Elsaman \& Arafa, 2012), we tested the hypothesis that, even for the non-relative targets, the intensity of gheirat is higher when the target is elderly (compared to youth).

Religiosity and traditionalism. We aimed to test the extent to which a person's religiosity can predict how much they are prone to experiencing gheirat, and how they define the concept. In addition to religiosity, we included traditionalism (i.e., the adherence to the national, 
but not necessarily Islamic, traditions) as a benchmark for comparison with religiosity. First, we tested the hypothesis that traditionalism and religiosity have a positive correlation with intensity of experiencing gheirat. Next, we tested whether one of these two elements are a stronger predictor of experiencing gheirat.

In Study 1, we documented an ambivalence in attitudes towards gheirat. Some participants perceived it as a virtue (e.g., protecting the vulnerable) while others saw it as a flaw (e.g., jealousy or malicious envy). Several religious texts on gheirat (e.g., Motahhari, 1968) have dedicated sections delineating how gheriat is different from hesadat (malicious envy), raising the possibility that the differences in attitude towards gheirat are related to participants' religiosity. As such, we tested the hypothesis that traditionalism and religiosity will have a positive correlation with perceiving gheirat as overlapping with protectiveness, and a negative correlation with perceiving gheirat as overlapping with malicious envy.

\section{Method}

\section{Participants}

The sample for this study was the same as Study 2.

\section{Procedure and Materials}

Big Five Inventory. After completing the Study 2's materials, participants completed a brief 15-item version of the Big Five Inventory-2 (Soto \& John, 2017). This scale was included for exploratory purposes and is further discussed in the Online Supplement.

Next, they were reminded that the remaining items are about their general experiences and attitudes towards gheirat, and unrelated to the previous section. They were then asked to report whether they have a sister and a brother. The responses to these questions were used when assigning different scenarios to participants (see "Scenarios with relative targets" below). 
Gheirat frequency. Participants reported the overall frequency with which they experience gheirat for 11 targets (e.g., "romantic partner") on a 4-point scale ( $1=$ never, $4=$ most of the time).

Scenarios with relative targets. Participants were given 26 scenarios for each of the three targets and asked to report the extent to which they experience gheirat in each scenario $(1=$ not at all, $5=$ extremely). First, all participants completed 26 scenarios with their romantic partner as the target. Next, they completed the same set of items once with a female relative as the target, and another time with a male relative as the target. Our aim was to investigate gheirat towards parents and siblings such that we can make gender comparisons within each category. Accordingly, for those participants who had indicated that they have both a sister and a brother ( $55 \%$ of the total sample), the targets in these scenarios were sister and brother. For others, the targets were mother and father.

For each target, of the 26 scenarios, 22 included a violation based on the three themes extracted from the first study. The scenarios were chosen so that they cover a wide range, from scenarios that only highly gheirat-prone people would react to (e.g., one's namoos shaking hand with a person of a different gender) to those that most people consider to be a prototypical gheirat-eliciting situation (e.g., namoos having an affair). In addition, four "neutral" scenarios, in which none of the gheirat-eliciting themes were present, were included as points of comparison.

Scenarios with non-relative targets. In response to 24 scenarios, participants were asked to report the extent to which they would experience gheirat $(1=$ not at all, $5=$ extremely $)$. These scenarios included non-relative targets (e.g., religion, nationality, country flag, favorite sports team). The common theme among these scenarios was an insult or harm to the target (e.g., "imagine a foreigner burns down the Iranian flag."). The characteristics of the target and the 
perpetrator were manipulated in three ways: (a) self-relevant threat vs. non-self-relevant threat:

For example, disrespect to the participants' nationality vs. disrespect to another nationality; (b) Ingroup perpetrator vs. outgroup perpetrator; and (c) domain of insult: consisting of religion, nationality, flag, job/career, sports team, gender, age, and political group.

To keep the number of items within a feasible range, we did not include the 'ingroup vs. outgroup perpetrator' comparison for all domains. Instead, based on the importance and cultural relevance of ingroup vs. outgroup comparison to each domain, we only included this comparison for religion, nationality, and flag burning. As such, there were four scenarios for each of these domains, in which the self-relevance of the threat and the group membership of the perpetrator was manipulated. For the other domains (i.e., job/career, sports team, gender, age, and political group), two scenarios were included in which the self-relevance of the threat was manipulated.

Religiosity and traditionalism. Two items were used to measure religiosity (e.g., "how religious do you consider yourself?" 1 = not at all religious, $5=$ deeply religious; $\alpha=.86$ ). Furthermore, to capture participants' adherence to Iranian cultural norms and traditions, we measured "traditionalism" using two items (e.g., "abiding by the traditional rules and rituals is very important to me"; 1 = completely disagree, $5=$ completely agree; $\alpha=.47$ ).

Overlap between gheirat, protectiveness, and malicious envy. Using two visual items inspired by the "inclusion of other in the self" scale (Aron et al., 1992), participants reported how much they believe gheirat overlaps with protectiveness and malicious envy $(1=$ no overlap, $5=$ complete overlap).

\section{Results}

As with Study 2, our overall aims were largely descriptive and exploratory. We preregistered some specific hypotheses but not a comprehensive set, and conceptually coherent 
groupings of results include both preregistered and non-preregistered ones. Therefore, for clarity of presentation, we again present analyses together without prioritizing preregistered analyses. All preregistered analyses are reported in the online supplement. Similar to Study 2, for nonpreregistered analyses, we use the alpha threshold of .005 for significance, and refer to $.05>p \geq$ .005 as "suggestive," as recommended by Benjamin et al., (2018). Data, analyses code and output reproducing the results are available at https://osf.io/pxsbv/?view_only=69c35a03c254409fabe213737077c9c6.

\section{General Frequency of Gheirat Toward Different Targets}

How frequently do people experience gheirat towards different targets, and does this vary as a function of gender? We asked participants to rate in general how often they experience gheirat toward 11 people and entities. A non-preregistered 11 (Targets) X 2 (Participant's gender) mixed factorial ANOVA revealed a significant difference among the targets $(F(10$, $\left.2880)=104.53, p<.001, \eta^{2}{ }_{p}=.27\right)$. As depicted in Figure 6, romantic partner, followed by mother, country, and sister were the targets towards which participants experienced the highest frequency of gheirat.

There was no significant main effect of gender $\left(F(1,288)=0.20, p=.650, \eta^{2}<.001\right)$. The ANOVA did reveal a significant Target X Gender interaction $(F(10,2880)=5.89, p<.001$, $\left.\eta_{p}^{2}=.02\right)$. Simple effects analyses showed only one significant difference: Women reported experiencing more gheirat towards their religion or beliefs compared to men, $M_{\text {diff. }}=0.39$, $t(1343)=3.50, p<.001, d=0.38$. There were three suggestive differences such that men (compared to women) reported higher frequency of gheirat towards their sister, their favorite sports team, and strangers $\left(0.23<M_{\text {diffs }}<0.27 ; 2.10<|t| \mathrm{s}<2.45 ; 0.22<d \mathrm{~s}<0.36\right)$. 


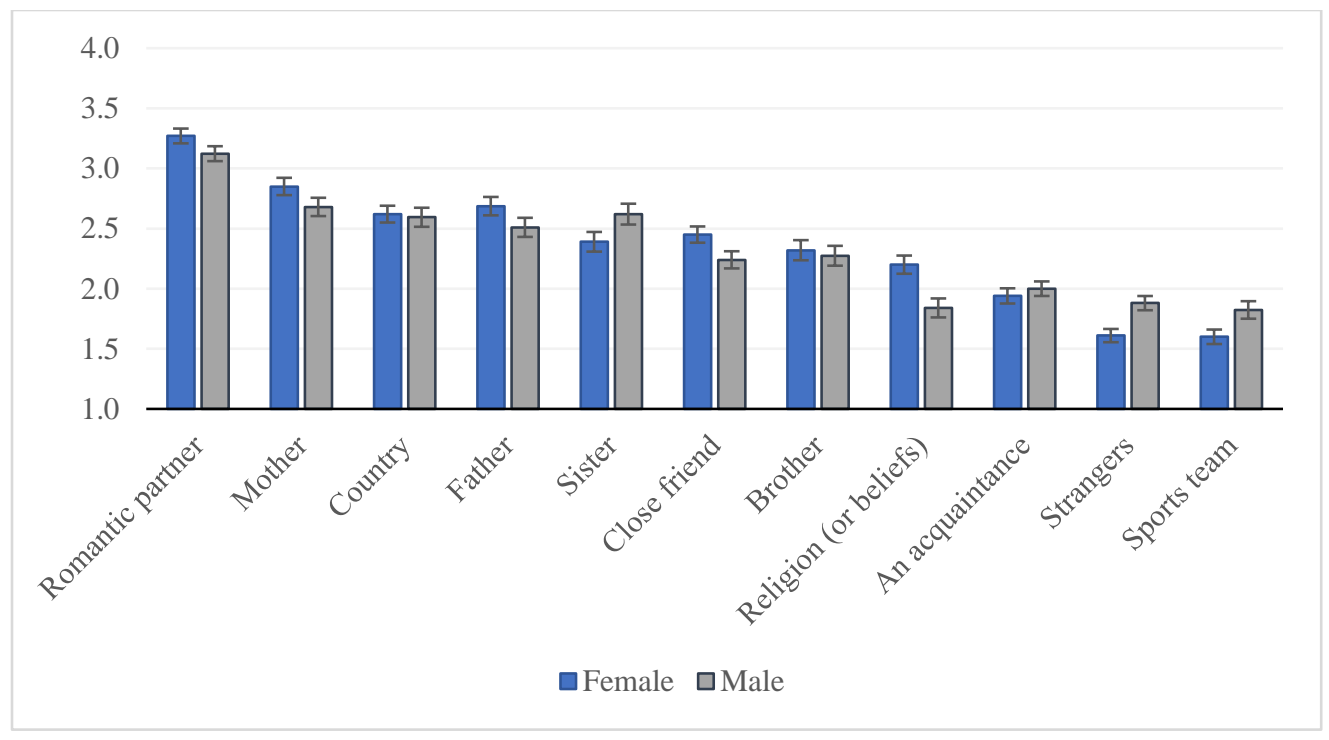

Figure 6. Frequency of experiencing gheirat for different targets.

\section{Gheirat Towards Relatives}

Participants rated the intensity of gheirat they would experience in 26 scenarios (22 theorized to be gheirat-eliciting and 4 neutral comparisons) for 5 different targets ( 3 per participant), resulting in 130 scenario ratings (78 per participant). We used PCA as a data-driven strategy to reduce the dimensionality of the scenarios. We then investigated how participant gender, target characteristics, and different types of elicitors influence the intensity of gheirat.

We conducted a non-preregistered PCA on the 26 scenarios for partners, for whom we had ratings from the entire sample ${ }^{5}$ and who appeared most often in gheirat-eliciting situations in Studies 1 and 2. (PCAs on other targets produced similar, though not identical, results). The PCA results (discussed in detail in the Online Supplement) indicated a five-component structure with the three first components mapping onto the previously discussed gheirat-elicitor types: (1) violation by other (eight items; e.g., "if a person of a different gender catcalls my partner on the

\footnotetext{
${ }^{5}$ To compare the responses of participants who did and did not have a partner, we conducted 26 exploratory independent-samples t-tests. There was no significant difference between the partnered and non-partnered participants' responses on any of the 26 partner scenarios ( $p s>.525$; see Table OS10 in the Online Supplement).
} 
streets"), (2) perception of harm or insult to namoos (three items; e.g., "if someone oppresses my partner or violates their rights"), and (3) violation by namoos (four items; e.g., "if my partner cheats on me and have sexual relationship with a person of a different sex"). The last two components consisted of scenarios in which the partner had interactions with a person of a different sex (component four; three items) or same sex (component five; four items) which were not considered a violation (e.g., "when my partner jokes around with a person of the same sex"). The five components had eigenvalues between 8.00 and 1.22, and cumulatively explained $67 \%$ of the total variance (see Figure OS6 for the screeplot and Tables OS8 and OS9 for item loadings and component statistics).

For each participant, five component scores were created by averaging the responses to the corresponding items. For all targets, the mean intensity of gheirat for the last two components were considerably lower than the first three components $\left(0.92 \leq\right.$ Cohen's $\left.d_{z} \mathrm{~s} \leq 3.86\right)$, suggesting that the last two components represent scenarios that are mostly perceived as neutral daily interactions which are unlikely to elicit gheirat; therefore, we did not include these components in the following multi-level model. Further details are reported in Table OS11.

Comparison of scenario types. To probe five exploratory questions about the intensity of gheirat elicited by different violations across different targets, as well as the moderating role of the participant's gender, we conducted a non-preregistered linear mixed effects model with random intercepts. The three predictors were Target (5 levels: partner, sister, mother, father, brother), Type of Violation (3 levels: violation by other, perception of harm, violation by namoos), and participant's gender; the intensity of gheirat was the outcome. Except for the main effect of gender $\left(F(1,366)=2.74, p=.099, \eta^{2} p=.007\right)$, all the main and interaction effects were significant $\left(F_{\mathrm{S}} \geq 4.20, p \mathrm{~s} \leq .001, .005 \leq \eta^{2} \mathrm{~s} \leq .380\right)$. To examine the following research 
questions, additional non-preregistered pair-wise comparisons and simple effects analyses were conducted:

(a) What is the difference between gheirat intensity towards different targets? Pairwise comparisons indicated that, averaging across the different boundary violations, the intensity of gheirat was highest when partner or mother were the targets, followed by father, sister, and brother. Except for the difference between gheirat towards partner and mother $(t(3065)=0.001$, $p=.999)$, all other differences were significant $(|t| \mathrm{s} \geq 3.23, p \mathrm{~s} \leq .001)$.

(b) Are there gender differences in gheirat towards each target? Simple effects analyses indicated that the gender difference in gheirat intensity towards partner, mother, and sister was not significant $(|t| \mathrm{s} \leq 1.84, p \mathrm{~s} \geq .066)$; however, women (compared to men) expressed stronger gheirat when the target was father $\left(\operatorname{adj} . M_{\text {diff }}=0.36, t(1073)=3.31, p=.001\right)$ or brother $(\operatorname{adj}$. $\left.M_{\text {diff }}=0.57, t(867)=5.73, p<.001\right)$.

(c) How does different boundary violations affect the intensity of gheirat? Averaging across targets, scenarios which involved a perception of harm or insult elicited the strongest gheirat, followed by violations by namoos, and violations by others ( $t \mathrm{~s} \geq 16.62, p \mathrm{~s} \leq .001)$.

(d) Are there gender differences in gheirat elicited by different boundary violations? Simple effects analyses did not reveal a significant gender difference for harm/insult scenarios $\left(\operatorname{adj} . M_{\text {diff }}=0.17, t(561)=1.94, p=.053\right)$ or scenarios that included a violation by other $(\operatorname{adj}$. $\left.M_{\text {diff }}=0.01, t(560)=0.17, p=.866\right)$. The results were suggestive that women (compared to men) might experience stronger gheirat when there is a violation by namoos $\left(\operatorname{adj} . M_{\text {diff }}=0.23, t(564)=\right.$ $2.68, p=.007)$.

(e) Does the intensity of gheirat elicited by different boundary violations vary for different targets? Pairwise comparisons revealed distinct patterns for each variant of gheirat. As 
demonstrated in Figure 7 and Table 4, in scenarios where there was a perception of harm, the difference between intensity of gheirat towards various targets was relatively small (marginal mean differences ranged from 0.06 to $0.44 ; M=0.23$ ) compared to scenarios where there was a violation by namoos (marginal mean differences ranged from 0.01 to $1.01 ; M=0.50$ ) or a violation by other (marginal mean differences ranged from 0.02 to $1.32 ; M=0.67$ ). These results suggest that the norms for gheirat towards different family members are more homogenous when there is a perception of harm or insult to namoos but vary considerably when there is a violation by namoos or a third person.

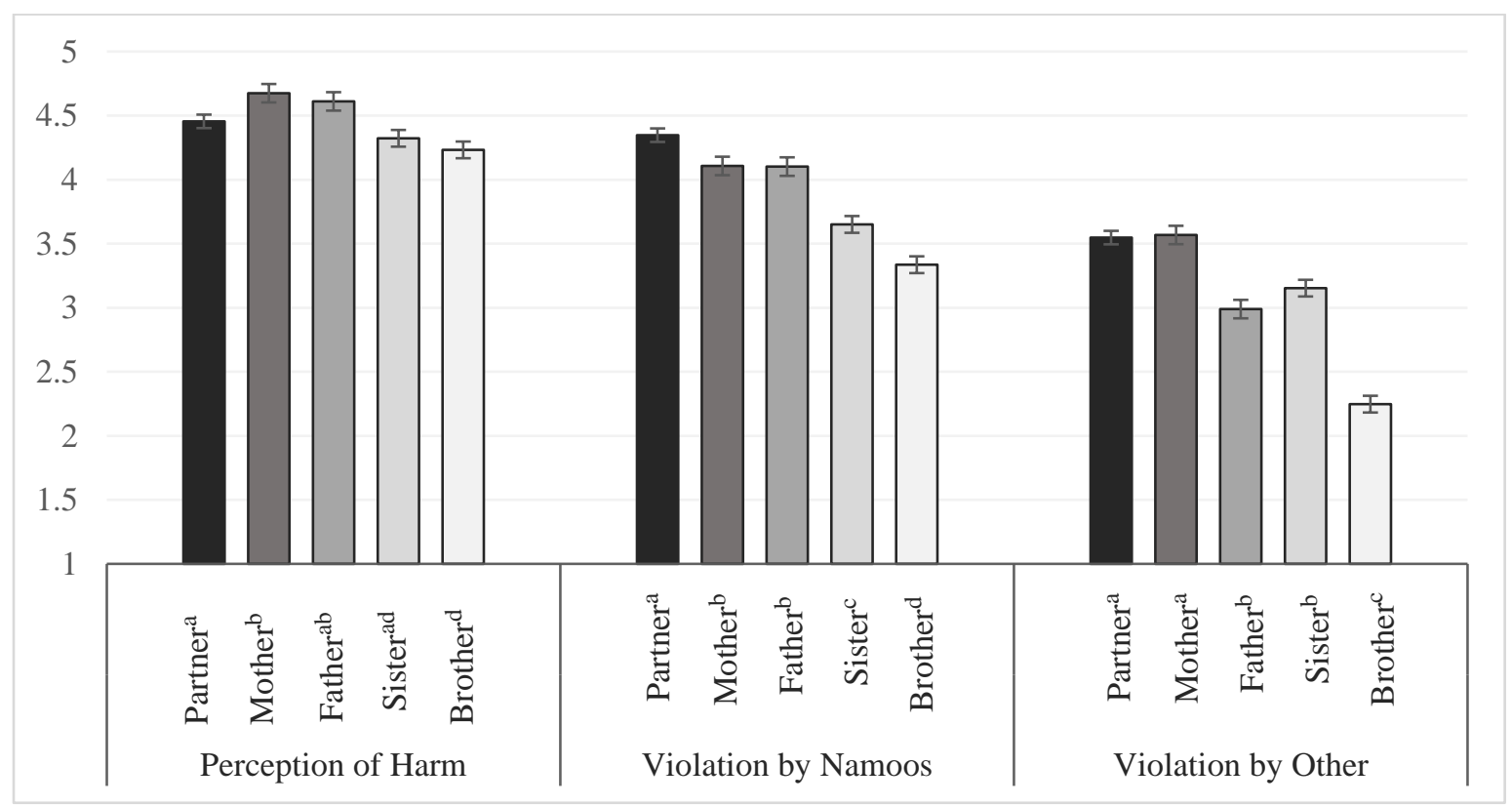

Figure 7. Estimated marginal means of different variants of gheirat towards relative targets.

Note. Within each type of violation, means that do not share a superscript letter are significantly different $(p s<.003)$. 
Table 4.

Gheirat Intensity for Each Target Across Different Scenario Types

\begin{tabular}{lccccc} 
& \multicolumn{5}{c}{ Scenario Type* } \\
\cline { 2 - 6 } Target & 1 & 2 & 3 & 4 & 5 \\
\hline Partner & $4.45^{\mathrm{a}}(0.72)$ & $3.53^{\mathrm{b}}(1.00)$ & $4.35^{\mathrm{a}}(0.80)$ & $1.68^{\mathrm{c}}(0.72)$ & $2.15^{\mathrm{d}}(1.00)$ \\
Sister & $4.35^{\mathrm{a}}(0.92)$ & $3.20^{\mathrm{b}}(1.08)$ & $3.69^{\mathrm{c}}(1.36)$ & $1.48^{\mathrm{d}}(0.52)$ & $1.63^{\mathrm{d}}(0.78)$ \\
Mother & $4.64^{\mathrm{a}}(0.67)$ & $3.50^{\mathrm{b}}(1.03)$ & $4.07^{\mathrm{c}}(1.06)$ & $1.43^{\mathrm{d}}(0.54)$ & $1.55^{\mathrm{d}}(0.76)$ \\
Brother & $4.24^{\mathrm{a}}(1.00)$ & $2.26^{\mathrm{b}}(1.03)$ & $3.34^{\mathrm{c}}(1.39)$ & $1.51^{\mathrm{d}}(0.58)$ & $1.40^{\mathrm{d}}(0.66)$ \\
Father & $4.60^{\mathrm{a}}(0.82)$ & $3.00^{\mathrm{b}}(1.10)$ & $4.09^{\mathrm{c}}(1.10)$ & $1.60^{\mathrm{d}}(0.73)$ & $1.92^{\mathrm{e}}(0.97)$ \\
\hline
\end{tabular}

Note. The estimates are means (and standard deviations). For each target, the means that do not share a superscript letter are significantly different at $p<.001$.

* (1) Perception of harm or insult, (2) Violation by other, (3) Violation by namoos, (4) Ordinary interactions with a person of same gender, (5) Ordinary interactions with a person of different gender.

\section{Insults to Religion, Nationality, and National Flag}

For each domain, we tested two preregistered hypotheses: (a) insults by an outgroup perpetrator elicits stronger gheirat, and (b) insults to self-relevant targets elicits stronger gheirat. To investigate these hypotheses, we conducted a preregistered 2 (perpetrator: ingroup vs. outgroup) X 2 (target: self-related vs. other-related) X 3 (domain of insult: religion, nationality, or flag) ANOVA with gheirat intensity as the dependent variable. As predicted, the main effects of perpetrator and target self-relevance were significant, indicating that insults by an outgroup (compared to ingroup) and insults to self-relevant (compared to other-relevant) targets elicits stronger gheirat $\left(F \mathrm{~s} \geq 25.19, \eta_{p}^{2} \mathrm{~s} \geq .006\right)$. We explored the main effect of domain of insult, which was significant $\left(F(2,3852)=267.37, p<.001, \eta^{2}=.12\right)$; non-preregistered analyses indicated that insults to nationality elicited the highest intensity of gheirat $(M=3.15)$, followed 
by burning of the national flag $(M=2.84)$ and insults to religion $(M=2.34$; $|t| \mathrm{s}>8.33$, $p \mathrm{~s}<$ $.001)$.

Since all the interaction effects in the pre-registered model were significant $(F \mathrm{~s} \geq 9.78, p \mathrm{~s}$ $\left.<.001, \eta_{p}^{2} \mathrm{~s} \geq .005\right)$, we followed up with non-preregistered tests of the effect of different perpetrators and target self-relevance for each of the three domains of insult separately (Figure 8). The results indicated a significant Perpetrator $\mathrm{X}$ Target interaction effect for all three domains. Importantly, simple effects analyses revealed that, in all domains, when the perpetrator was an outgroup, insults to self-related targets elicited significantly more gheirat compared to insults to other-related targets $\left(|t| \mathrm{s} \geq 6.42, p \mathrm{~s} \leq .001, d_{z} \mathrm{~s} \geq 0.32\right)$, but this was not the case when the perpetrator was an ingroup member. Only in the case of burning the flag by an in-group member, insult to self-related target elicited significantly stronger gheirat compared to insult to other-related target, $\left(t(1050)=-10.68, p<.001, d_{z}=0.51\right)$.

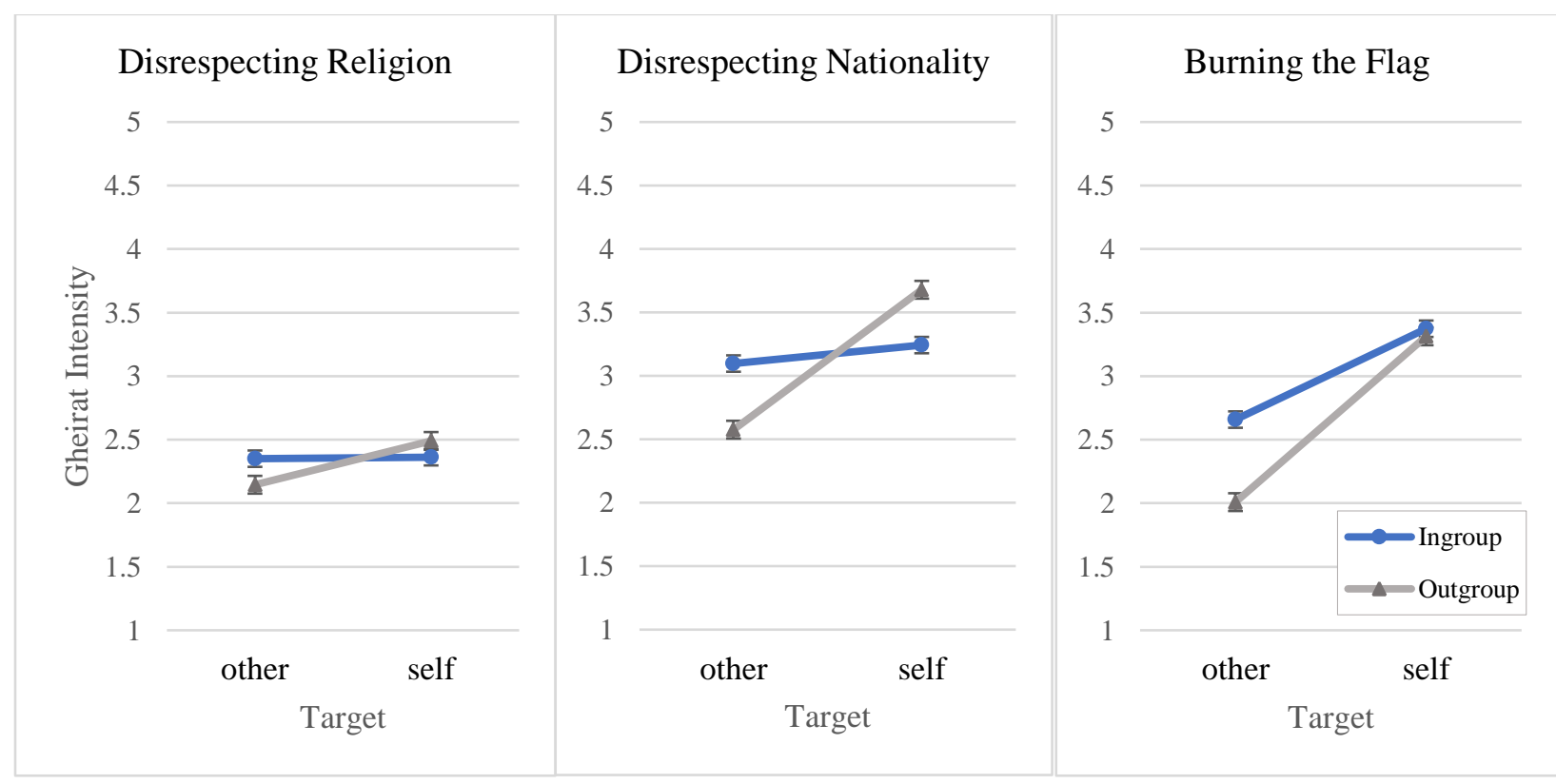

Figure 8. Gheirat in response to insults to religion, nationality, and national flag.

Note. Dark blue lines (with circular links) represent Perpetrator: Ingroup; grey lines (with triangular links) represent Perpetrator: Outgroup. 


\section{Insults to Career, Political Party, Gender, and Favorite Sports Team}

Within these domains, we tested the preregistered hypothesis that insults to self-relevant (compared to other-relevant) targets leads to higher intensity of gheirat using a preregistered 2 (target self-relevance) X 4 (domain of insult) ANOVA. (Note that we did not vary ingroup vs. outgroup for these domains.) As predicted, there was a significant effect of target $(F(1,2413)=$ $\left.386.88, p<.001, \eta_{p}^{2}=.136\right)$ such that stronger gheirat was elicited when the target of the insult was self-relevant.

\section{Insults to Gender and Age Groups}

We tested the preregistered hypotheses that insults to women (compared to men) and the elderly (compared to the youth) will elicit a higher intensity of gheirat. Both hypotheses were supported (for gender: $t(352)=12.63, p<.001, d_{z}=0.68$; for age group: $t(353)=7.56, p<.001$, $\left.d_{z}=0.40\right)$

To examine whether gheirat elicited in response to insults to gender and age groups are moderated by participants' own gender and age, we tested two non-preregistered interaction effects. A 2 (Target of insult: men vs. women) X 2 (Participant gender) ANOVA revealed a significant interaction effect $\left(F(1,350)=68.72, p<.001, \eta^{2}{ }_{p}=.16\right)$. Insults to women (compared to men) elicited a much stronger gheirat response among female participants $\left(d_{z}=1.04\right)$ compared to male participants $\left(d_{z}=0.32, p \mathrm{~s}<.001\right)$, suggesting that the higher level of gheirat in response to insults to women is primarily driven by female participants. However, similar interaction effect was not present for age $\left(F(1,351)=2.60, p=.107, \eta^{2}=.007\right)$.

\section{Relationship with Religiosity and Traditionalism}

To examine the role of religiosity and adherence to cultural traditions in participants' tendency to experience gheirat, a series of non-preregistered Spearman's non-parametric correlation tests were conducted. As represented in Figure 9 and Table OS14, two important 
patterns were present in these results. First, correlations with religiosity (mean of Spearman's $\rho \mathrm{s}$ $=.32)$ were on average stronger than correlations with traditionalism $\left(M_{\rho \mathrm{s}}=.16\right)$. Follow-up partial correlational analyses indicated that the relationships between religiosity and gheirat intensity, after controlling for traditionalism, either remained the same or became only slightly weaker $\left(M_{\rho \mathrm{s}}=.28\right)$, while the correlations between traditionalism and gheirat intensity, after controlling for religiosity, became much weaker, and mostly close to zero $\left(M_{\rho s}=.05\right)$. Overall, the pattern of results suggests that religiosity (compared to adherence to cultural traditions) has a distinct relationship with gheirat intensity.

Second, of the three gheirat variants, gheirat elicited in response to perceptions of harm had the weakest relationship with religiosity (for violations by other: $M_{\rho \mathrm{s}}=.40$; for perception of harm: $M_{\rho \mathrm{s}}=.16$; for violations by namoos: $M_{\rho \mathrm{s}}=.39$ ). Similar results were observed for traditionalism. These results suggest that appraisal of the non-harm scenarios where there are violations by namoos or another person depends on how religious (and to a lesser extent, how traditional) a person is. (For more details, refer to Table OS14.)

Finally, we tested the preregistered hypothesis that traditionalism and religiosity will have a positive correlation with perceiving gheirat as overlapping with protectiveness, and a negative correlation with perceiving gheirat as overlapping with malicious envy. As predicted, more religious people believed that gheirat has more overlap with protectiveness $(\rho=0.26, p<$ $.001)$ and less overlap with malicious envy $(\rho=-0.20, p<.001)$. The relationships were descriptively weaker for traditionalism ( $\left.\rho_{\text {protectiveness }}=0.17, p=.001 ; \rho_{\text {envy }}=-0.08, p=.121\right)$. Together, these results support the notion that people's attitudes towards gheirat is influenced by their religiosity, such that those who are more religious define gheirat in a more positive light 
(i.e., protectiveness) while those lower in religiosity perceive gheirat as more negative (i.e., malicious envy).

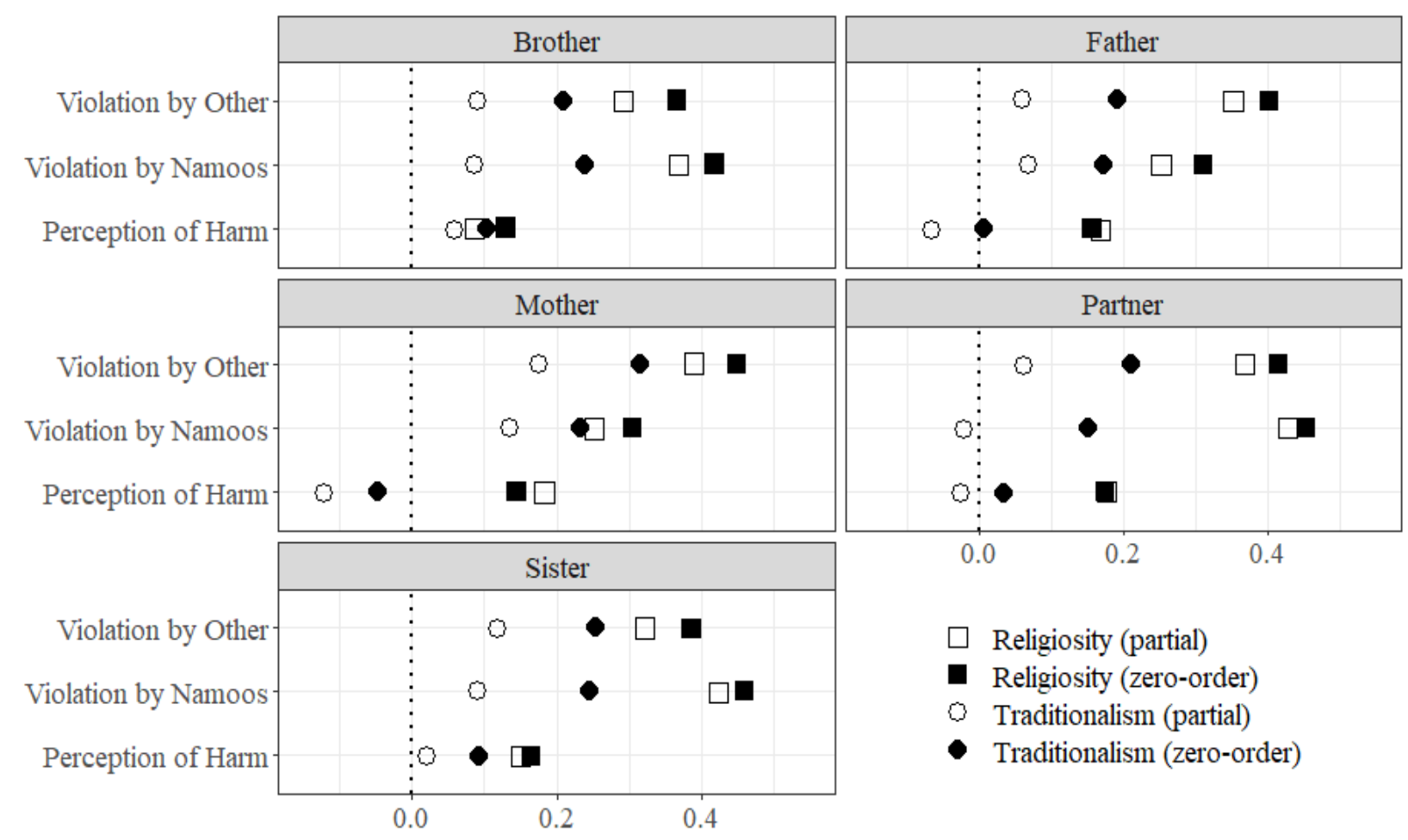

Figure 9. The correlations between religiosity, traditionalism, and gheirat towards different targets. X-axes represent Spearman's $\rho$ coefficients. For confidence intervals, see Table OS14.

\section{Discussion}

Results from this study corroborated those from the previous two in showing that three types of violations elicit gheirat. Of these three variants, the highest intensity of gheirat was elicited when there was a perception of harm or insult to namoos. An interesting pattern across analyses was that the gheirat response to harm or insult tended to be less dependent on the characteristics of the target (e.g., gender, relationship with participant) or the perceiver (e.g., religiosity, traditionalism). For the other two variants, however, intensity of gheirat varied depending on who the target is: partner, mother, and sister elicited the highest intensity. It also depended on the perceiver's religiosity: religious people experienced a higher intensity of 
gheirat. Traditionalism, which in this context meant endorsement of cultural (but not necessarily religious) norms and traditions, was not distinctively associated with gheirat intensity.

Regarding insult to non-relative entities (e.g., country or religion), as hypothesized, we found that violations against self-relevant entities (e.g., one's country) elicits stronger gheirat. This corroborates the notion of namoos as an extended network of self-relevant entities.

Furthermore, results indicated that the intensity of gheirat is higher when the perpetrator is an outgroup, a finding that has important implications for inter-group dynamics. Interestingly, the pattern of interaction between these two main effects suggests that inter-group insults, regardless of whether the target is part of the namoos, are perceived as a violation.

Finally, the results across various domains did not support the folk notion that gheirat is a primarily male experience. There was no considerable gender difference in the gheirat frequency towards different targets. In some cases, in fact, women experience higher intensity of gheirat compared to men. For example, while men and women had the same intensity of gheirat towards their partner and mother, women (compared to men) reported higher intensity when gheirat involved their brother or father. Overall, these results point out the complexity of the relationship between gender and gheirat, a topic that will be addressed further in the next study.

\section{Study 4}

Mentions of gheirat in religious and historical texts demonstrate that it is a key cultural feature that has been sustained generation after generation. What accounts for this sustained prominence of gheirat? And what are its social functions in Iranian culture? One approach to answering these questions, often adopted in social-functionalist accounts of emotions (e.g., Fischer \& Manstead, 2008; Frijda \& Mesquita, 1994), is to investigate the social consequences 
of experiencing and expressing this emotion-laden phenomenon by studying how Iranians interpret one another's displays of gheirat.

From a social-functionalist approach, scripts and norms about emotional experiences and their expression are deeply embedded in the culture (Keltner \& Haidt, 1999). Communities regulate their members' adherence to norms through reputational mechanisms such as character judgments (Craik, 2009). In the case of gheirat, there are indications that deviating from such norms can be socially costly. For example, during the interviews, participants perceived the motive to save face and protect one's reputation as deeply intertwined with the experience of gheirat. Gaining a reputation as someone who is not prone to gheirat can harm a person's social standing and make them targets of humiliation (e.g., Abedinifard, 2019). In the present study, our overarching goal is to investigate the social impressions that the expression of gheirat (or lack thereof) leaves on perceivers, and how it may benefit or harm relationships by changing the expressor's reputation and value as a desirable target for social affiliation.

Results from Studies 1 and 3 suggest an ambivalence in attitudes towards gheirat: gheirat-proneness is sometimes admired as a virtue and sometimes perceived as an undesirable trait akin to proneness to jealousy or malicious envy. This ambivalence is consistent with Atari et al.'s (2020) findings that people who endorse gheirat-related values tend to engage in both socially desirable (i.e., benefit provisioning) and socially undesirable (i.e., cost inflicting) mate retention strategies (Atari et al, 2020). Accordingly, we predicted that a person who expresses gheirat will be perceived positively in some domains (e.g., as brave) and negatively in others (e.g., as stubborn). To capture these nuances, we measured the social consequences of expressing gheirat using a constellation of positive and negative moral and character judgements developed 
based on the literature on virtues (Park et al., 2006), the findings from Study 1, and the results of a preliminary study (explained later).

In addition to our primary goal, which was to investigate judgements of a person who expresses gheirat, we had three other research questions:

1. Does the gheirat expresser's gender affect the social consequences? It is a common perception that expectations about expressing gheirat are highly dependent on gender (KhezrHeidari, 2018). Across the Study 1 interviews, participants told us that men, more than women, are expected to experience and express gheirat. However, in Studies 2 and 3, which focused on elicitors and phenomenology, we found few gender differences. Could it be that gender differences reside more in the domain of social judgment? In the present study we experimentally manipulated the gender of the protagonist and examined the gender $\mathrm{X}$ expressivity interaction. If it is the case that gheirat is expected of men, but not women, social consequences of expressing gheirat should be moderated by gender, as a woman who expresses gheirat is perceived as violating social norms.

2. Are the social consequences specific to expressing gheirat? There are social consequences of showing vs. suppressing expressive behavior for a wide range of emotions (Butler et al., 2003; Gross \& John, 1998; Tackman \& Srivastava, 2016). Because our hypotheses were specific to gheirat, we wanted to rule out general expressivity effects as an alternative explanation. To do that, in addition to the comparisons between a person who expresses vs. does not express gheirat, we added a third condition in which the target expresses sadness, another emotion that would be socially acceptable during the studied scenarios (based on results of the preliminary study). By comparing the judgments of a protagonist who expresses gheirat and one who expresses sadness, we can rule out general expressivity effects. 
3. Some religious texts on gheirat refer to it as an unconditional virtue (Mazaheri, 2016; Tabatabaei, 1976), similar to the notion of sacred values (Dehghani et al., 2010), devotion to which is unconditionally admired. In contrast, the broader literature on emotional reactions suggests that expression of emotions often follow culturally accepted scripts (Keltner \& Haidt, 1999; Tsai et al., 2006), deviations from which are perceived as a norm violation and judged negatively. In Study 4, we assigned participants to different conditions in which expressing gheirat was either expected or unexpected. This allows us to test whether gheirat is perceived as an unconditional virtue, judged regardless of the situation, or if the social consequences of gheirat dependent on the context.

\section{Method}

\section{Preliminary Study}

A preliminary study $\left(N=182,53.3 \%\right.$ female, $\left.M_{\text {age }}=33.56\right)$ was conducted to (a) pilot test and select scenarios in which expression of gheirat or sadness is expected, and (b) develop a list of virtues and character judgements relevant to gheirat expressivity. For details, including the justification for scenario and character judgment selection, see the Online Supplement.

\section{Participants}

In our preregistration, we estimated the sample size needed to test our main research questions (e.g., "comparing the character evaluations of a man who expresses gheirat vs. does not") aiming for a medium population effect size $(\delta=.50)$ and power of .95 at $\alpha=.05$ (twotailed) to be a minimum of 105 participants per cell; as such we aimed for a minimum sample size of 630 participants. Participants learned about the study through announcements on the Telegram social network platform and received personality feedback after completion of the study. The final sample consisted of 694 Iranian adults $\left(M_{\text {age }}=31.76, S D_{\text {age }}=8.75,50.9 \%\right.$ 
female). Of the participants who responded to additional demographics questions, $89.3 \%$ reported residing in Iran, $86.9 \%$ reported that they have had a romantic partner in the past, and $65.6 \%$ reported having a romantic partner now. Of the participants who reported their education, $42.1 \%$ were undergraduate students or had a bachelor's degree, $48.3 \%$ were graduate students or had a graduate degree, and the remaining $(9.7 \%)$ had a high school diploma, pre-university, or an associate degree. Participants reported low to moderate levels of religiosity $(M=2.00, S D=$ 1.12, on a 1-5 scale). Sensitivity analyses (Faul et al., 2009) indicate that this final sample size $(N$ $=694)$ has $80 \%$ power $(\alpha=.05$, two-tailed $)$ to detect small-to-moderate effect sizes for withinsubjects mean differences $\left(d_{z}=0.11\right)$, between-subjects mean differences $(d=0.21)$, and withinbetween interaction effects in a 2 X 2 mixed ANOVA $(f=0.06)$. Preregistration and study materials are available at https://osf.io/f38m7, and data, analyses code and output reproducing the results are available at https://osf.io/pxsbv/

\section{Procedure and Materials}

The study was designed and administered in Persian/Farsi. After providing consent, participants read a total of two scenarios. For the first, participants were assigned to read one of six scenarios in which the protagonist's gender and their expressivity were manipulated based on a 2 (protagonist's gender: male or female) X 3 (expressive reaction: gheirat vs. sadness vs. no expression) design. These scenarios were designed so that the protagonist was expected to experience and express gheirat. The protagonist's gender was conveyed through their name, which was randomly selected from a list of eight popular names in Iran. The following scenario is an example with a male protagonist who reacts by expressing gheirat. The parts that are in bold were changed based on the experimental condition: 
Amir is in the car with his spouse, and his spouse is driving. They get into a car crash, and the other driver insults the spouse and curses at her. Amir feels gheirat, gets out of the car, and gives a firm warning to the offensive driver. (Sadness condition: "Amir feels sad, gets out of the car, and expresses his sadness to the offensive driver." No expression condition: "Without showing any emotional reaction, Amir gets out of the car and talks to the driver about repairing the car.”)

Character judgements. After reading the scenarios, participants were asked to evaluate the protagonist on 26 character judgments and four statements about their desire to affiliate with the protagonist. Items were rated on a 5-point Likert scale (Strong disagree to Strongly agree). We preregistered a PCA on the character judgments, which we ran as a data-driven strategy to reduce the dimensionality of the character judgments prior to conducting the main analyses. Results indicated that these character judgments can be reduced to four components: Competence (7 items, $\alpha=.88$; e.g., "wise" and "intellectual"), Obstinance ( 5 items, $\alpha=.81$; e.g., "stubborn" and "numskull/fatuous"), Warmth (5 items, $\alpha=.79$; "kind" and "grateful"), and Virtue (4 items, $\alpha=.70$; “virtuous/pious" and "noble/genteel”). The four components had eigenvalues ranging from 8.81 to 1.42 (see Figure OS12 for the screeplot) and cumulatively explained 58\% of the total variance (see Tables OS16 and OS17). Accordingly, for each participant, four scale scores were created by averaging the responses to the corresponding items. Desire to affiliate. Participants responded to four statements regarding their attitudes towards or desire to affiliate with the protagonist (e.g., "I'd like to be friends with someone like [protagonist's name]"). Items were rated on a 5-point Likert scale (not at all to a lot). Following the preregistration, since these four items were highly correlated (inter-correlations ranging from $r=.65$ to .86$)$, we averaged them to create a composite score for affiliation $(\alpha=.91)$. 
Next, participants read a second scenario in which the protagonist's gender and their expressive reaction was the same as the first scenario. However, in this scenario, experiencing and expressing gheirat was unexpected (the socially expected reaction was sadness). For example:

Niloofar is in the car with her spouse, and the spouse is driving. She receives a call on her phone and the caller informs her that a family member died after a long illness. Niloofar feels sad and continues talking with the caller in a sad tone. (Gheirat condition: "Niloofar feels gheirat and continues talking with the caller in a harsh tone." No expression condition: "Without showing any emotional reaction, Niloofar continues talking to the caller about the funeral ceremonies.")

After reading the second scenario, the participants rated the protagonist's character and their desire to affiliate with the protagonist using the same 26 items as discussed earlier.

\section{Results}

We ran a preregistered 3 (Expression) X 2 (Protagonist's gender) X 2 (Expectancy) mixed factorial ANOVA for each character judgement scale, as well as the affiliation component. Considering the important role of gender in perceptions of gheirat, the following preregistered contrasts examine the primary research questions for male and female targets separately:

\section{Effects of Expressing Gheirat}

Our primary research question was about the effects of expressing vs. not expressing gheirat when it is socially appropriate and expected. Planned contrast analyses indicated that in such situations, a man who expresses gheirat is perceived as being more obstinate $(\beta=0.64$, $t(1276)=7.09, p<.001)$ and more $\operatorname{warm}(\beta=0.64, t(1230)=7.31, p<.001)$ than a man who 
does not express gheirat. Furthermore, participants expressed a stronger desire to affiliate with a man who expresses gheirat $(\beta=0.43, t(1323)=3.76, p<.001)$. Effect of expressivity on competence and virtue were non-significant $(|\beta| \mathrm{s} \leq 0.14,|t| \mathrm{s} \leq 1.56, p \mathrm{~s} \geq .113)$.

The pattern of results was different when the protagonist was a woman (Figure 10). Planned contrast analyses indicated that a woman who expressed gheirat was seen as less competent $(\beta=-0.73, t(1298)=-8.00, p<.001)$, more obstinate $(\beta=0.96, t(1276)=10.43, p<$ $.001)$, more warm $(\beta=0.40, t(1230)=4.52, p<.001)$, and less virtuous $(\beta=-0.21, t(1254)=-$ $2.89, p=.004)$ than a woman who did not express gheirat. Additionally, participants expressed less interest in affiliation with a woman who expressed (vs. did not express) gheirat ( $\beta=-0.25$, $t(1323)=-2.20, p=.028)$.

To directly compare the previous results for men and women, we tested a series of planned interaction contrasts. These analyses revealed a significant Gender X Expressivity interaction for all judgments except warmth. The effect of expressing gheirat on competence judgements was more negative when the protagonist was a woman $(\beta=-0.59, t(1298)=-4.60, p$ $<.001)$. The effects on perceptions of obstinacy were more strongly positive for women $(\beta=$ $0.32, t(1276)=2.45, p=.014)$. For virtue, the effect of expressing gheirat was more negative for women $(\beta=-0.32, t(1254)=-3.17, p=.002)$. Finally, the effects on preference for affiliation were opposite for women and men $(\beta=-0.68, t(1323)=-4.21, p<.001)$. 

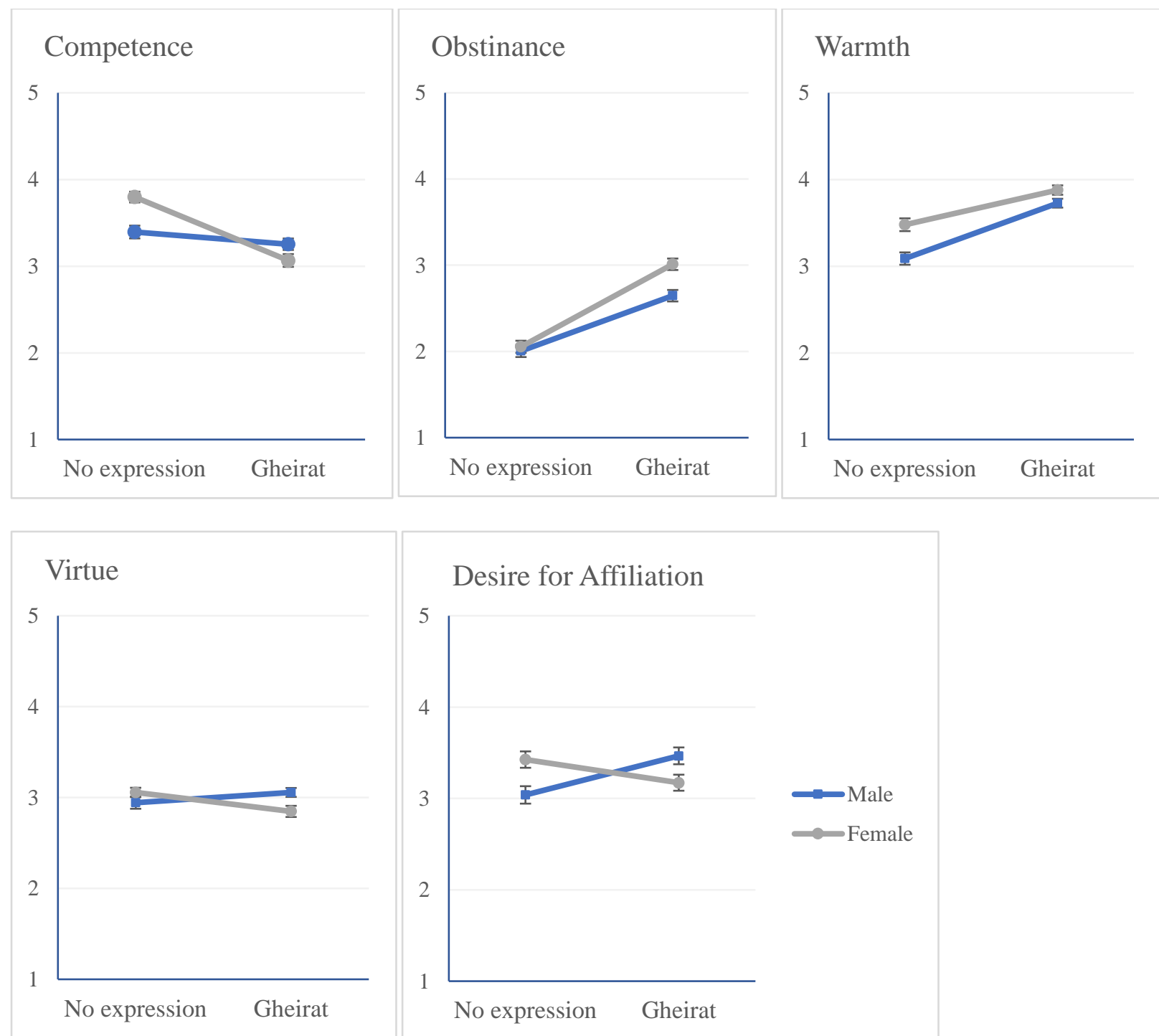

Figure 10. A comparison of character judgements about and desire to affiliate with a protagonist who expresses gheirat (vs. no expression) when it is expected.

\section{Comparison to Sadness}

Are these evaluations specific to gheirat, or are they a result of general expressivity? If the effect of expressing gheirat on social judgments (as seen in the previous section) is a simple expressivity effect, we would expect no difference between judgments of a person who expresses gheirat and someone who expresses sadness. Planned simple contrast analyses indicated that, in a 
situation where gheirat is expected, a man who expresses gheirat (as opposed to sadness) is seen as less competent $(\beta=-0.22, t(1298)=-2.50, p=.013)$ and more obstinate $(\beta=0.41, t(1276)=$ 4.60, $p<.001)$. However, there was no difference in the rating of warmth and virtue $(|\beta| \mathrm{s} \leq 0.10$, $|t| \mathrm{s} \leq 1.41, p \mathrm{~s} \geq .160)$. Furthermore, participants reported more interest in affiliation with the man who expressed gheirat (as opposed to sadness; $\beta=0.23, t(1323)=2.10, p=.036$ ). The pattern of results was similar for the female protagonist, except for affiliation ratings. A woman who expressed gheirat (compared to sadness) was judged as less competent $(\beta=-0.26, t(1298)=-$ $2.90, p=.004)$ and more obstinate $(\beta=0.27, t(1276)=3.01, p=.003)$. However, there was no difference in the ratings of warmth, virtue, and participants' desire to affiliate with the protagonist $(|\beta| \mathrm{s} \leq 0.11,|t| \mathrm{s} \leq 1.02, p \mathrm{~s} \geq .306)$.

Direct comparison of the results for men and women via planned interaction contrasts revealed a significant gender X expressivity (gheirat vs. sadness) interaction only for participants' desire to affiliate and interact with the target, where the effect for men differed from that for women $(\beta=-0.34, t(1323)=-2.16, p=.031)$. Overall, the observed differences suggest that expressing gheirat (instead of another situationally acceptable emotion, sadness) has a number of distinct social consequences and cannot be reduced to a general expressivity effect.

\section{Effect of Expectancy on Perceptions of Gheirat}

Is expression of gheirat evaluated unconditionally (i.e., regardless of the situation), or is it considered a virtue or a vice depending on whether it is socially appropriate and expected? To examine this question, a series of preregistered 2 (gheirat expression vs. no expression) X 2 (gheirat expected vs. not expected) interaction contrasts with the different character judgments as the outcomes were conducted for male and female protagonists. For the male protagonist, results revealed significant interaction effects for all outcomes $(-0.42 \leq \beta \mathrm{s} \leq 1.53,-3.79 \leq t \mathrm{~s} \leq 11.28, p \mathrm{~s}$ 
$<.001)$. Overall, the pattern of results indicated that evaluations of gheirat for men are strongly context-dependent: Expressing gheirat when it is not expected (vs. when it is expected) leads to stronger negative evaluations (i.e., obstinance) and weaker positive evaluations (i.e., competence, warmth, and virtue) of men's character. Furthermore, participants expressed a very low desire to affiliate with a man who expresses gheirat when it is not expected. Similar interaction effects were observed for female targets for most outcomes $(0.21 \leq \beta \mathrm{s} \leq 0.80,2.34 \leq$ $t \mathrm{~s} \leq 6.92, p \mathrm{~s} \leq .019)$, except for obstinance $(\beta=0.11, t(679)=0.97, p=.334)$. Overall, results demonstrated that for women, while the expression of gheirat in general is often judged unfavorably, the negative consequences are even stronger if gheirat is expressed when it is not expected. (For detailed results, see Online Supplement.)

\section{Mediators of the Desire to Affiliate with the Target}

What impressions does the expression of gheirat leave that can lead to a desire to affiliate more or less with the expresser? To investigate this question, we ran two planned mediation models (one for each gender category) with expressivity $(0=$ no expression, $1=$ gheirat $)$ as the predictor, desire to affiliate as the outcome, and the four character judgements as the mediators. As demonstrated in Figure 11, for the male target, we found evidence for possible mediation of obstinance $(\beta=-0.17, z=-2.88, p=.004)$ and warmth $(\beta=0.25, z=3.16, p=.002)$ : a man who expressed gheirat (vs. no expression) was perceived as more obstinate, which was associated with a weaker desire to affiliate with him, but was also perceived as more warm, which was associated with a stronger desire to affiliate with him. In contrast, for the female target, results supported the possible mediation of competence $(\beta=-0.41, z=-5.13, p<.001)$ and warmth $(\beta=$ $0.19, z=3.21, p=.001)$ : a woman who expressed gheirat was perceived as more warm, which was associated with a stronger desire to affiliate with her; but at the same time she was perceived 
as much less competent, which was associated with a weaker desire to affiliate with her. In both models, Virtue was not a significant mediator ${ }^{6}$.

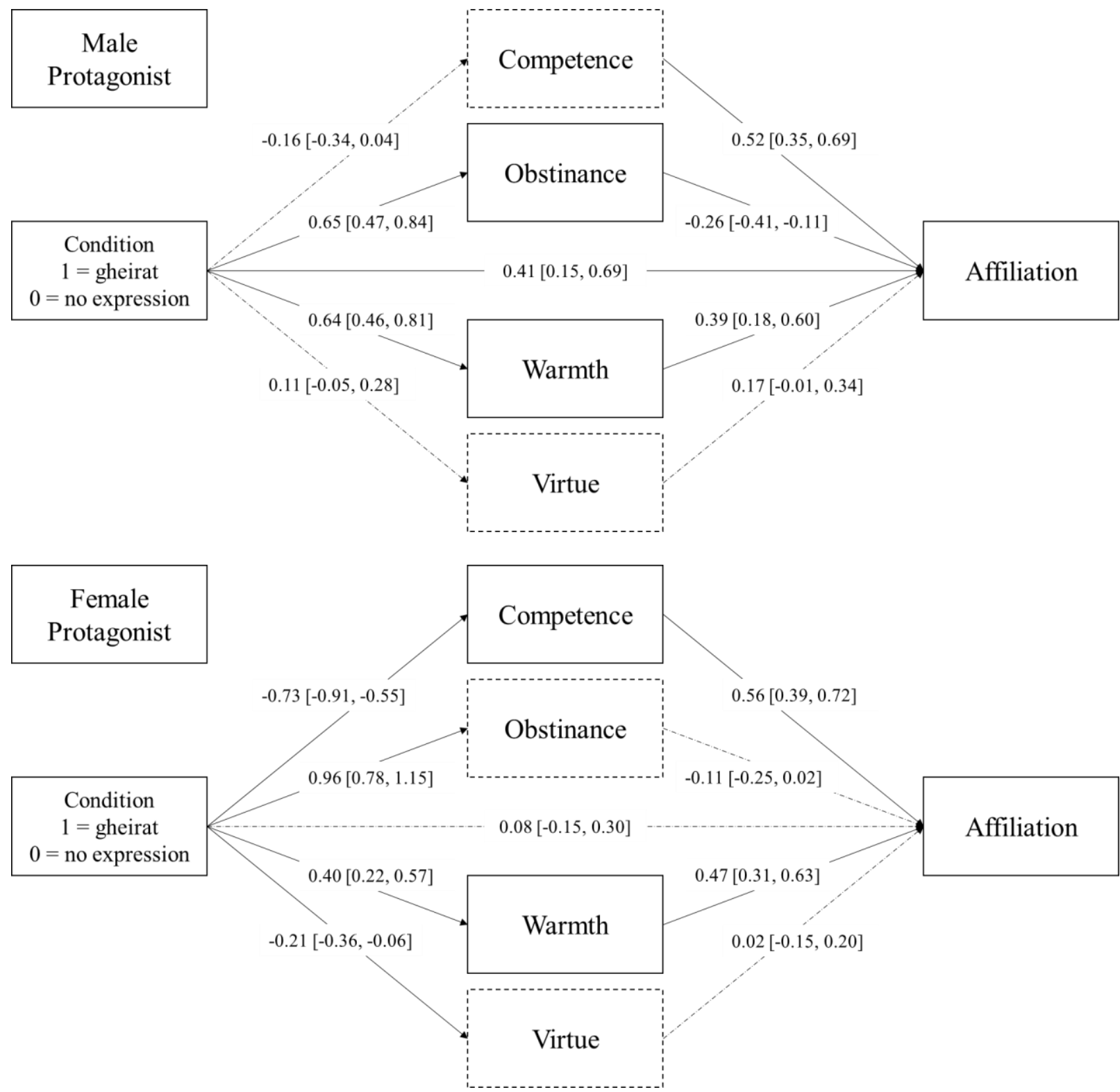

Figure 11. Path analyses indicating the mediators of the effect of gheirat expression on participants' desire to affiliate with the expresser.

Note. Values on the paths are $\beta$ s with lower- and upper-boundary 95\%CIs in the brackets. Solid arrows indicate significant $\beta$ s and solid squares indicate significant mediators $(p \mathrm{~s} \leq .008)$.

${ }^{6}$ Exploratory mediation analyses in which the four character judgments that were used to calculate the Virtue component were individually entered as mediators (instead of the Virtue composite score) demonstrated that none of these character judgments were significant mediators of the effect of gheirat expression on desire to affiliate with the protagonist. 
These results suggest that for both men and women, a constellation of positive and negative evaluations, together with other mechanisms not measured here, may help explain the effects of expressing gheirat on others' desire to affiliate. However, men and women differ in which judgments matter, and whether the positive or negative ones prevail.

\section{Discussion}

Social evaluations of a person who expresses gheirat consist of a combination of positive and negative evaluations, and they are conditional on the expressor's gender and the situational expectancy. In a social interaction where expressing gheirat is expected, a man who expresses gheirat is perceived as both warmer and more obstinate than a man who does not. A woman who expresses gheirat is also judged as warmer and more obstinate than a woman who does not, but she is also perceived as less competent. As a whole, the positive evaluations resulting from expressing gheirat are stronger for men, and the negative ones are stronger for women. Importantly, a man who expresses gheirat is perceived as a more desirable person to affiliate with, whereas the opposite happens for a woman who expresses gheirat. These results suggest that the expression of gheirat can be socially beneficial for men but costly for women.

Results from Studies 2 and 3 did not support the folk theories about gheirat being a primarily male experience. Tying those results to the findings from this study, one possible conclusion is that the gender difference in gheirat is not about the internal experience of gheirat, but how it is expressed in observable behavior and whether it is socially acceptable. This is consistent with results from Study 2 that demonstrated that, during a gheirat episode, women were more likely to engage in expressive reactions that could be interpreted as sadness (e.g., crying) or masking of the negative emotion (e.g., smiling), whereas men were more likely to display overt aggressive intentions. If the social norms make expressions of gheirat costly for 
women, they may choose to mask their emotional reaction or express it in a way that it would not be interpreted as gheirat. On a societal level, this might lead to a collective perception that men (but not women) experience gheirat.

\section{General Discussion}

We started this program of research with the overarching question, "What is gheirat?" Through four studies, using mixed methods and an emic-etic approach, we investigated the phenomenology of gheirat through Iranians' perspective, documented similarities and variations in various components of the experience, investigated situational elicitors and individual differences, and studied its reputational effects. Here, we synthesize the findings and discuss their implications.

\section{What is gheirat?}

A prototypical experience of gheirat entails an emotional response to relational boundary violations that involve namoos. The eliciting event is generally perceived as unfair, unexpected, and improper or immoral. Participants' appraisals of gheirat-eliciting situations indicate that, prototypically, they have a strong sense of responsibility towards namoos, together with a mild sense of insecurity and low sense of self-worth. Breaking down the affective components of the prototypical gheirat, we see that it involves relatively strong hostility and moderate levels of socially fearful emotions. Not surprisingly, participants evaluate the experience as unpleasant and detrimental to their self-esteem and their relationships, and their expressive and behavioral responses to a gheirat episode involves negative hostile expressions with a tendency towards verbal aggression.

In search of variants of gheirat, we found three types of situations that elicit gheirat. Although all three types of gheirat elicitors embody an element of threat, our findings suggest 
that different types of boundary violations lead to diverse emotional and behavioral responses (Figure 12). In fact, we see considerable differences between gheirat in response to harm and insults and gheirat in response to boundary violations by namoos - the former is marked by stronger aggressive tendencies, the latter entails a preference to withdraw from others. This is a theoretically important finding when thinking about situating the current results within models of personality and the broader literature on honor, which we will discuss later.

\section{Gheirat and Personality}

The tendency to experience an emotion (e.g., fear) can be perceived as a personality trait (e.g., fear-proneness; Saucier \& Iurino, 2020). In religious texts and popular discourse, terms such as gheirati (gheirat-prone) or bigheirat (not gheirat-prone) are regularly used to describe a person's personality or character. This is consistent with the findings from a psycho-lexical study of personality structure based on Persian/Farsi lexicon (Herfehdoust et al., 2015) where researchers found gheirat proneness to emerge as one of the indicators of a culture-specific dimension of personality. Furthermore, results from Study 4 suggest that signaling gheiratproneness can provoke a wide range of personality and character evaluations about a person. These insights highlight the cultural significance of gheirat-proneness as a personality trait and has important implications for models of personality. 


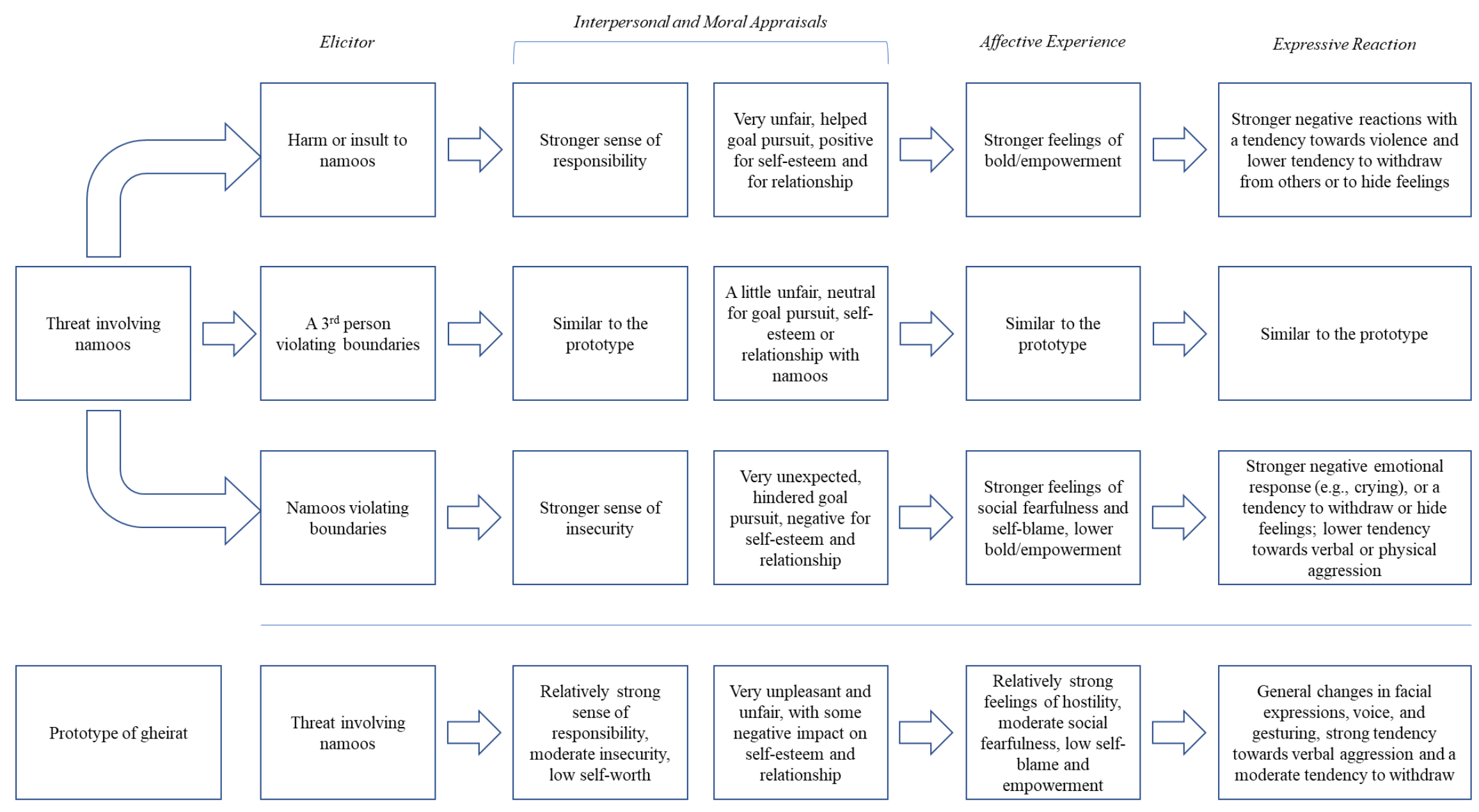

Figure 12. Differences in appraisals, affective experiences and expressive reactions associated with different variants of gheirat. 


\section{Gender and Gheirat}

Deeply embedded within the folk theories of gheirat is the idea that gheirat is primarily a male experience. However, in Study 1 we found that women were able to describe personal experiences of gheirat, and Studies 2 and 3 showed many similarities between men and women in the intensity, frequency, and phenomenology of gheirat. Considering the widespread belief about gender differences in gheirat, this might sound like a puzzling finding. However, gender differences in behavioral responses might provide relevant clues. Results from Study 2 suggest that men and women express gheirat differently. A higher proportion of women reported expressive responses that could be interpreted as masking the experience of gheirat with another negative emotion (e.g., crying, sobbing) or even a positive one (e.g., smiling). It is possible that even though men and women often experience gheirat with similar intensity, women's tendency to suppress or mask their expressions leads to a general perception that gheirat is a male experience.

Why would women suppress or mask their expression of gheirat? The findings from Study 4 may offer an answer. Gheirat-expressers are judged positively on some dimensions (e.g., warmth) and negatively on others (e.g., obstinance). This effect, however, is strongly moderated by the expresser's gender, such that the positive evaluations are stronger for men and negative ones are stronger for women. Importantly, men who express gheirat are perceived as a more desirable target of affiliation, whereas women are perceived as less desirable. In the presence of such gender double-standards, it is plausible that women use masking or expressive suppression to avoid negative social evaluations. The perceived gender differences, therefore, are not necessarily in the fundamental experience of gheirat, but in how men and women manifest their experiences outwardly to navigate the social norms. The present findings provide some early 
insights about the gender dynamics in relation to gheirat and highlight the complexities of this topic. For example, although most analyses of intensity and frequency of gheirat did not indicate gender differences, there were differences in the proportions of elicitors when participants spontaneously wrote about a time in the past they experienced gheirat in Study 2. Although men and women wrote about all three themes in non-negligible numbers, women's narratives were relatively more likely to include boundary violation by namoos, whereas men's narratives more often included the other two themes. These nuances highlight the need for future studies focused on gender differences (or lack thereof) in the meaning, experience, and expression of gheirat.

\section{Who is Gheirat-Prone?}

Gheirat is an important part of religious conceptions of the virtuous Muslim, and in line with that we found that those who are more religious are more prone to experiencing gheirat. This relationship, however, was not the same across different variants of gheirat. Gheirat elicited in response to perceptions of harm had the weakest relationship with religiosity. By contrast, gheirat elicited by boundary violations (either by namoos or by an outsider) were more strongly associated with religiosity. This fits with the religious texts' emphasis on gheirat as a way to enforce socio-sexual norms on female relatives and partners. Less religious Iranians seem to care less about those elicitors, but are still sensitive to harm and insults to namoos ${ }^{7}$.

Interestingly, there were some indications that the meaning of gheirat might vary depending on a person's religiosity. More religious participants were more likely to see an

\footnotetext{
${ }^{7}$ Apart from religiosity, we also conducted exploratory analyses on the relation between Big Five personality traits and the experience of gheirat. Due to the psychometric limitations of our short Big Five scale, we decided to include the detailed results in the Online Supplements. Briefly, these results revealed that two personality traits often associated with social self-regulation (i.e., conscientiousness and agreeableness) were positively correlated with gheirat-proneness. Surprisingly, the results did not suggest a significant relationship between gheirat-proneness and traits associated with conservatism (i.e., openness) or negative affect (i.e., neuroticism). These findings are consistent with Atari et al.'s (2020) results about the relation between Big Five and gheirat-related attitudes.
} 
overlap between gheirat and protectiveness and less likely to see an overlap between gheirat and malicious envy. A potential underlying mechanism for these differences may be the way religious beliefs influence people's perceptions of relationships. For example, a more religious person may consider close social interactions between their namoos and a person of different gender as a "fertile ground" for temptation and sin. Consistently, gheirat-related behaviors or attitudes that restrict such interactions would be interpreted as "protecting" the namoos from committing a sin. In contrast, a less religious person who does not subscribe to such beliefs about relationships may interpret the same restrictive behaviors and attitudes as a manifestation of malicious envy. Considering the importance of the relation between religiosity and gheirat, unpacking the underlying processes of this relation would be an important subject for future research.

\section{Gheirat and Honor}

As we began this program of research, we purposely took an emic approach so that our investigation would not be constrained by theories and assumptions developed in other cultural settings. Instead, after developing an understanding of gheirat through this approach, we can situate it within the prior psychological literature. We focus here on honor, a well-studied phenomenon with particular relevance to gheirat.

(a) Honor threats. Are the situations that elicit gheirat among Iranians similar or different to those identified as honor violations in research conducted in other settings? The answer was complex. Many violations that elicit gheirat would be recognizable to people from other cultures, especially those with an emphasis on honor norms. One area of similarity is in behavior that is perceived as an explicit harm or insult, which was one of the major variants of gheirat and is documented as honor-bound hostility in other honor cultures studied across the 
world (e.g., Cohen et al., 1996; Guerra et al., 2013; Günsoy et al., 2020a, IJzerman et al., 2007).

The other two variants (gheirat elicited by socio-sexual norm violations by namoos or a third person) had elements that were more dependent on the religious norms that are specific to this cultural context. Although the underlying theme in these variants is similar to some of the gendered norms of honor documented in other non-Muslim cultures (e.g., Rodriguez Mosquera et al., 2002), some situational concerns such as sensitivity to clothing, any form of contact (e.g., shaking hands) between non-family members of different genders, or female namoos being at home or work with a person of different gender are directly related to Muslim religious principles such as hijab and khalwa that regulate or forbid such instances. Their relevance to gheirat depends on culturally specific concepts like namoos and mahramiat (Moradinasab, 2021), and they may not be seen as a serious violation in some non-Muslim honor cultures (e.g., the southern United States).

(b) Honor-related emotions. Honor threats often elicit strong emotions; as such, there is a tradition in honor literature to measure emotional reactions as a signal for honor norms. For example, one of the widely used measures of honor (Rodriguez Mosquera et al., 2002; Rodriguez Mosquera, 2016) asks participants to report how bad they would feel about themselves in different situations. Many of these situations revolve around violations that would elicit gheirat in an Iranian (e.g., “you were unable to defend your family's reputation”). People from many cultural backgrounds may describe their emotional reaction to these norm violations with broad language such as "feeling bad." In a Muslim cultural context where concerns about protecting these relational boundaries are highly prioritized, related concepts become hypercognized in language (Levy, 1984; Russell, 1991a). As a result, a single, specific word - "gheirat" - has emerged to describe the complex cluster of feelings elicited by these highly prioritized boundary 
violations. By using or referencing this emotion word (e.g., saying "I felt gheirat"), people can communicate critical social and personal information (e.g., the situation that elicited their experience, the phenomenology of the experience) that are more specific and culturally relevant than broader emotion concepts (e.g., "I felt bad" or "I felt angry").

Focusing on gheirat as an emotional signal of honor violations sheds light on the way honor threats are clustered together in this cultural context. We see a clear distinction between gheirat-eliciting situations based on the way the violation is appraised (i.e., insult/harm vs. sexual/relational) and the relationship with the violator (namoos vs. a third person). Notably, deconstructing the emotional components of gheirat demonstrates that the affective response to boundary violations is more nuanced than "feeling bad." For example, when there is a perception of harm or insult, the experience consists of a mixture of specific negative (e.g., hostility) and positive (e.g., bold/empowered) emotional experiences. Future research using a more granular measurement of emotion can determine whether these emotional configurations are culturespecific or if they appear in other honor cultures.

(c) Reputation. Reputation is a core component of honor (Pitt-Rivers, 1965). Similarly, gheirat experiences have a strong connection with reputation. Two of the gheirat-related appraisal categories in Study 2, insecurity and self-worth, reflect participants' concerns about their social standing. Results from Study 4 extend this idea and demonstrate how expressing gheirat can be socially beneficial (or costly) to one's reputation. Notably, the present findings show the importance of the negative and positive reputational consequences in gheirat experiences. In Study 2, participants' appraisals of the gheirat episode reflected both negative (e.g., "I felt my reputation is at risk") and positive reputational consequences (e.g., "I felt respected"). These two clusters of appraisals emerged as two distinct components that are mildly 
correlated $(r=-.27)$, suggesting that the positive and negative reputational consequences are not simply two poles of a single continuum, and deserve discrete attention. This is further corroborated by the Study 4 findings demonstrating that gheirat expressors are not only perceived as someone who is unyielding and holds grudges, they are also judged as warmer (e.g., more kind, caring, and dependable). This is an important insight for applied research on gheirat (e.g., in marital relations), where a holistic understanding of positive and negative reputational motives can facilitate addressing maladaptive gheirat-related behavior.

(d) The positive side of honor. There has been considerable attention to the negative consequences associated with honor, especially in relation to violence, aggression, and revenge (see Thrasher \& Handfield, 2018, and Uskul et al., 2019), leading to an abundance of studies focused on the detrimental consequences of honor norms (e.g., Altheimer, 2013; Foster, 2020; Osterman \& Brown, 2011). However, multidimensional conceptualizations of honor (e.g., Cross et al., 2014; Rodriguez Mosquera et al., 2002) highlight the importance of a more nuanced perspective that incorporates positive components, such as the desire to be socially responsible and behave morally. The present findings are in agreement with this perspective: Beyond hostility, gheirat involves a sense of responsibility and obligation, especially towards collective values and those who are vulnerable. In Study 2, the strongest appraisal category associated with gheirat was the sense of responsibility following someone being oppressed or a boundary being violated. Importantly, this responsibility extends to those who are perceived as vulnerable, even if they are not immediate family members or ingroups (Study 3). These findings point to the positive role a gheirat-prone person can play for the collective as a deterrent against aggressors, and are consistent with some of the recent accounts about the functionality of honor agents in societies (e.g., Nowak et al. 2016; Sommers, 2018). Positive consequences are not limited to 
collective benefits: at a personal level, in gheirat experiences in response to harm or insult, participants experienced a boost to their self-esteem and their relationship, and considered the experience helpful to their goal pursuit (Study 2). Inspired by these findings, we believe that the theoretical conceptualizations of gheirat can benefit from a nuanced attention to the negative and positive aspects of people's experiences, especially as this research extends into applied domains such as mental health care (see Gul et al., 2021).

\section{Applications}

As the global pattern of immigration leads to increasing diversity in Western countries (Besharov \& Lopez, 2016; "Migration to Europe," 2018; Radford, 2019), there is a growing need for a better understanding of cultural dynamics that can affect intergroup interactions. In the absence of accurate knowledge about outgroups, people are likely to use stereotypes and develop prejudiced attitudes (Matusitz, 2012). Research suggests that Westerners are prone to misinterpreting Muslims’ motives and emotions (Bijlstra et al., 2010; Kommattam et al., 2017), and therefore perceiving and labeling them negatively (Verkuyten, \& Zaremba, 2005) — a trend that can have considerable negative effect on inter-cultural interactions and Muslim immigrant's assimilation (Bruneau et al., 2020; Kamans et al., 2009). High profile controversies in the media (e.g., the ban of burkini in France (Breeden \& Blaise, 2016) and the Muslim students in Switzerland refusing to shake hands with their teachers (Bachmann \& Onyanga-Omara, 2016; Taylor, 2016)) reflect the inter-group tension fueled by different cultural values. As we demonstrated in the present research, some of the situations or behaviors which are perceived as norm violations that elicit gheirat are unfamiliar to those from non-Muslim cultures. Furthermore, the negative consequences of some of these norm violations are exacerbated when the violator is an outgroup member. Consequently, we believe that the present findings about the 
dynamics of gheirat provides applicable insights for social, political, and educational contexts in which those associated with Muslim and non-Muslim cultural backgrounds interact.

\section{Limitations and Future Directions}

In interpreting the present findings, several limitations should be taken into account. First, the present research focused on the everyday experience of gheirat. Events that are more rare or extreme, such as honor killings or extremist violence, were outside of the present scope but would be ripe for future investigation.

Second, our samples were recruited from one Muslim-majority country, Iran. People who share a religion share many cultural norms and values, even across national boundaries (White et al., 2021), and both anecdotal evidence and scholarly writings suggest gheirat is a key cultural component in a wide range of Muslim-majority countries. However, it is important to be mindful of the heterogeneity among Muslim-majority countries, particularly in relation to ethnic and linguistic differences (e.g., Arab and non-Arab), geographic locations (e.g., Middle East vs. Southeast Asia), different denominations (e.g., Shi'a and Sunni), or non-Muslim religious minorities. Another limitation of this work is that we did not ask about sexual and gender minority status, which could be associated with potentially substantial differences in the experience of gheirat. The extent to which the present findings generalize across other Muslim sub-cultures, and potentially extend to non-Muslim cultures, is an empirical question for future research. In extending this research to cultural contexts where there is no direct linguistic equivalent for gheirat, there is an opportunity to broaden our understanding of hypocognition and constructionist views of emotions by investigating how a lack of conceptual knowledge about gheirat affects related psychological processes (e.g., Wu \& Dunning, 2019) and what are the 
effects of introducing gheirat to people who are not familiar with this concept (e.g., Hoemann et al., 2022).

Finally, as discussed extensively in the introduction, due to the novelty of the research topic in the psychological literature, our approach was a mixture of hypothesis generating and hypothesis testing. With regard to the former, our positionality was both an aid in understanding this topic and a potential source of gaps in perspective. With regard to the latter, many of the results are based on non-preregistered, data driven analyses; some, but not all, were followed up with preregistered tests. Readers should be mindful of this distinction (Srivastava, 2018) when interpreting the results and judging how definitive our conclusions are. Future work in this area could take greater advantage of preregistration, including for qualitative methods (Haven \& Van Grootel, 2019).

The insights generated from the present research can be expanded in several ways. First, the unexpected findings regarding gender and gheirat suggests that this important aspect of gheirat requires further interrogation. Future research, building on the present insights, can provide a more focused explanation of the societal dynamics that contribute to the discrepancies between folk theories of gheirat and the present findings. Mixed-methods approaches, such as sequential explanatory designs (Tashakkori \& Teddlie, 1998; Creswell et al., 2003) where quantitative data are followed by qualitative data within one study are particularly well-suited for such explanatory goals.

Second, the three variants of gheirat in this research emerged from three themes that emerged across diverse methodologies. Future research can investigate sub-themes (e.g., different types of harm, insult, or sexual norm violations) and different characteristics of the 
situation (e.g., participants' sense of closeness with the namoos, private vs. public violations) to further explore the variability in emotional and behavioral components of gheirat.

Finally, the present research begins to describe the signaling function of gheirat through the study of social perceptions of gheirat expressors. There are various possibilities for extending these findings. For example, the misalignment between emotional experience and expressivity (Greenaway \& Kalokerinos, 2019) is a dimension that was not controlled or manipulated in Study 4. It is plausible that a protagonist who does not express gheirat (but is experiencing it) is perceived differently from one who does not express gheirat (and is not experiencing it).

Furthermore, since one of the primary goals of Study 4 was to assess the social consequences of expressing gheirat on a wide range of character judgments, we limited the number of scenarios to avoid participant fatigue and attrition. Although we used themes from the Study 2 narratives and the findings from a pilot study to ensure the selected scenarios are typical and relatable cultural experiences, it is possible that some of the findings are specific to the selected scenarios. Future extensions of this study where the experience-expression dimensions are experimentally manipulated, and participants' judgments are evaluated across a greater variety of scenarios can extend the boundaries of the present findings. 


\section{References}

“Abedi: Nuclear energy technology was achieved by the perseverance of the Iranian nation”. (2011, April, 9). IRNA. https://www.irna.ir/news/9233733/

Abedinifard, M. (2019). Persian 'Rashti jokes': Modern Iran's palimpsests of gheyrat-based masculinity. British Journal of Middle Eastern Studies, 46(4), 564-582.

"About Majid Shahriari: Scientist and theoretical designer of the early generation of nuclear reactors”. (2019, November, 27). IRNA. https://www.irna.ir/news/83569590/

Abu-Lughod, L. (1999). Veiled sentiments. Honor and poetry in a Bedouin society. Berkeley, CA: University of California Press.

Akbari, D., \& Tetreault, P. (2014). Honor killing: A professional's guide to sexual relations and ghayra violence from the Islamic sources. Bloomington, IN: AuthorHouse.

Al-Bukhari (1976). Sahih Al-Bukhari. Kazi Publications, Chicago, IL (translated by Muhammad Muhsin Khan).

Allamezade, S. (2016). Men writing women: "The Woman question” and male discourse of Iranian modernity [Doctoral dissertation, University of Maryland]. https://drum.lib.umd.edu/handle/1903/18601

Altheimer, I. (2013). Cultural processes and homicide across nations. International Journal of Offender Therapy and Comparative Criminology, 57(7), 842-863.

Ando, H., Cousins, R., \& Young, C. (2014). Achieving saturation in thematic analysis: Development and refinement of a codebook. Comprehensive Psychology, 3(4).

Arshad, M., \& Chung, J. M. (2022). Practical recommendations for considering culture, race, and ethnicity in personality psychology. Social and Personality Psychology Compass, 16(2), e12656. 
Aron, A., Aron E. N., \& Smollan, D. (1992). Inclusion of other in the self scale and the structure of interpersonal closeness. Journal of Personality and Social Psychology, 63, 596-612.

Asch, S. E. (1987). Social psychology. New York: Oxford University Press. (Original work published 1952)

Ashokkumar, A., \& Swann Jr, W. B. (2022). Restoring Honor by Slapping or Disowning the Daughter. Personality and Social Psychology Bulletin. Advance online publication. https://doi.org/10.1177/01461672221079106

Aslani, S., Ramirez-Marin, J., Brett, J., Yao, J., Semnani-Azad, Z., Zhang, Z. X., ... \& Adair, W. (2016). Dignity, face, and honor cultures: A study of negotiation strategy and outcomes in three cultures. Journal of Organizational Behavior, 37(8), 1178-1201.

Atari, M. (2018). Culture of Honor. In Zeigler-Hill, T. K. Shackelford (eds.), Encyclopedia of Personality and Individual Differences (pp. 1-4). New York: Springer International Publishing.

Atari, M., Barbaro, N., Shackelford, T. K., \& Chegeni, R. (2017). Psychometric evaluation and cultural correlates of the Mate Retention Inventory-short form (MRI-SF) in Iran. Evolutionary Psychology, 15(1), 1474704917695267.

Atari, M., Graham, J., \& Dehghani, M. (2020). Foundations of morality in Iran. Evolution and Human Behavior, 41, 367-384.

Atari, M., \& Jamali, R. (2016). Mate preferences in young Iranian women: Cultural and individual difference correlates. Evolutionary Psychological Science, 2(4), 247-253.

Auerbach, C., \& Silverstein, L. B. (2003). Qualitative data: An introduction to coding and analysis. New York University Press.

Averill, J. R. (1980). A constructivist view of emotion. In R. Plutchik \& H.Kellerman (Eds.), 
Emotion: Vol. 1. Theory, research, and experience (pp. 305-340). New York, NY: Academic Press.

Azarbaijani-Moghaddam, S. (2012, April, 23), Manly honor and gendered male Afghanistan. The Middle East Institute (MEI). https://www.mei.edu/publications/manly-honor-andgendered-male-afghanistan

Baboli, M. F., \& Karimi-Malekabadi, F. (2020, October 10). Qeirat Values and Victim Blaming in Iran: The Mediating Effect of Culture-Specific Gender Roles. https://doi.org/10.31234/osf.io/dvz9k

Bachmann, H., \& Onyanga-Omara, J. (2016, May 25). Muslim students face \$5K fine if they refuse Swiss teachers' handshakes. USA Today. https://www.usatoday.com/story/news/world/2016/05/25/swiss-authorities-overuleteacher-handshake-ban/84899900/

Bakhtiar, M. (2015). Cognitive model of gheirat in Persian. Cognitive Linguistic Studies, 2(2), 257-288.

Barakatullah, F. (n.d.). Reviving our sense of gheerah. Zawaj. http://www.zawaj.com/articles/reviving_gheerah.html

Barnes, C. D., Brown, R. P., \& Osterman, L. L. (2012). Don't tread on me: Masculine Honor Ideology in the U.S. and militant responses to terrorism. Personality and Social Psychology Bulletin, 38, 1018-1029.

Barnes, C. D., Brown, R. P., Lenes, J., Bosson, J., \& Carvallo, M. (2014). My country, my self: Honor, identity, and defensive responses to national threats. Self and Identity, 13(6), 638662.

Barrett, L. F., Quigley, K. S., Bliss-Moreau, E., \& Aronson, K. R. (2004). Interoceptive 
sensitivity and self-reports of emotional experience. Journal of Personality and Social Psychology, 87, 684-697.

Bastani, H. (2016, January, 25). “About Hosein Kordmihan, the person who ordered the attack on Saudi Arabia's embassy". BBC.

https://www.bbc.com/persian/iran/2016/01/160125_139_file_hassan_kordmihan_saudi_e mbassi

Becker, J. C., Tausch, N., \& Wagner, U. (2011). Emotional consequences of collective action participation: Differentiating self-directed and outgroup-directed emotions. Personality and Social Psychology Bulletin, 37(12), 1587-1598.

Behravesh, M. (2015). Iran's Nuclear Behavior: The Gender Dimension. Your Middle East. https://lucris.lub.lu.se/ws/portalfiles/portal/6008845/5471037.pdf

Benjamin, D. J., Berger, J. O., Johannesson, M., Nosek, B. A., Wagenmakers, E. J., Berk, R., ... \& Johnson, V. E. (2018). Redefine statistical significance. Nature Human Behaviour, 2(1), 6-10.

Berry, J. W. (2013). Achieving a global psychology. Canadian Psychology/Psychologie Canadienne, 54(1), 55-61.

Besharov, D. J., \& Lopez, M. H. (2016). Adjusting to a world in motion: Trends in global migration and migration policy. Oxford Scholarship Online.

Bijlstra, G., Holland, R. W., \& Wigboldus, D. H. (2010). The social face of emotion recognition: Evaluations versus stereotypes. Journal of Experimental Social Psychology, 46(4), 657663.

Boyatzis, R. E. (1998). Thematic analysis and code development: Transforming qualitative information. London and New Delhi: Sage Publications. 
Brady, L. M., Fryberg, S. A., \& Shoda, Y. (2018). Expanding the interpretive power of psychological science by attending to culture. Proceedings of the National Academy of Sciences, 115(45), 11406-11413.

Braun, V., \& Clarke, V. (2006). Using thematic analysis in psychology. Qualitative research in psychology, 3(2), 77-101.

Breeden, A., \& Blaise, L. (2016, August 12). Cannes, citing security risks, bans full-body 'burkinis' from its beaches. New York Times. https://www.nytimes.com/2016/08/13/world/europe/cannes-muslims-burkini-ban.html

Brislin, R. W. (1970). Back-translation for cross-cultural research. Journal of Cross-Cultural Psychology, 1(3), 185-216.

Bruneau, E. G., Kteily, N. S., \& Urbiola, A. (2020). A collective blame hypocrisy intervention enduringly reduces hostility towards Muslims. Nature Human Behaviour, 4(1), 45-54.

Butler, E. A., Egloff, B., Wlhelm, F. H., Smith, N. C., Erickson, E. A., \& Gross, J. J. (2003). The social consequences of expressive suppression. Emotion, 3(1), 48-67.

Clore, G. L., \& Ortony, A. (1991). What more is there to emotion concepts than prototypes? Journal of Personality and Social Psychology, 60, 48-50.

Cohen, J. (1988). Statistical Power Analysis for the Behavioral Sciences. New York, NY: Routledge Academic.

Cohen, D., Nisbett, R. E., Bowdle, B. F., \& Schwarz, N. (1996). Insult, aggression, and the southern culture of honor: An" experimental ethnography." Journal of Personality and Social Psychology, 70(5), 945-960.

Corbin, J., \& Strauss, A. (2008). Basics of qualitative research: Techniques and procedures for developing grounded theory ( $3^{\text {rd }}$ ed.). Thousand Oaks, CA: Sage. 
Coulson, N. (2017). A history of Islamic law. New York: Routledge.

Craik, K. H. (2009). Reputation: A network interpretation. New York, NY: Oxford University Press.

Creswell, J. W. (2013). Qualitative inquiry and research design: Choosing among five approaches. Thousand Oaks, CA: Sage.

Creswell, J. W., Plano Clark, V. L., Gutmann, M., \& Hanson, W. (2003). Advanced mixed methods research designs. In Handbook on mixed methods in the behavioral and social sciences, ed. Tashakkori, A. \& Teddlie, C., 209-40. Thousand Oaks, CA: Sage.

Cross, S. E., Uskul, A. K., Gerçek-Swing, B., Sunbay, Z., Alözkan, C., Günsoy, C., ... Karakitapoğlu-Aygün, Z. (2014). Cultural prototypes and dimensions of honor. Personality and Social Psychology Bulletin, 40, 232-249.

Dehghani, M., Atran, S., Iliev, R., Sachdeva, S., Medin, D., \& Ginges, J. (2010). Sacred values and conflict over Iran's nuclear program. Judgment and Decision Making, 5(7), 540-546.

Dhami, S., \& Sheikh, A. (2000). The Muslim family: predicament and promise. Western Journal of Medicine, 173(5), 352-356.

DiCicco-Bloom, B., \& Crabtree, B. F. (2006). The qualitative research interview. Medical Education, 40(4), 314-321.

Dorrough, A. R., \& Glöckner, A. (2016). Multinational investigation of cross-societal cooperation. Proceedings of the National Academy of Sciences, 113(39), 10836-10841.

Ekman, P., \& Cordaro, D. (2011). What is meant by calling emotions basic. Emotion review, 3(4), 364-370.

Ellsworth, P. C., \& Scherer, K. R. (2003). Appraisal processes in emotion. In R. J. Davidson, K. R. Scherer, \& H. H. Goldsmith (Eds.), Handbook of affective sciences (pp. 572-595). 
Oxford University Press.

Elsaman, R. S., \& Arafa, M. A. (2012). The rights of the elderly in the Arab Middle East: Islamic theory versus Arabic practice. Marquette Elder's Adviser, 14(1), 1-53.

Estiri, Z. (2019). How does the concept of honor develop over time in a typical honor oriented society: Iran. [Unpublished master's thesis]. Utrecht University.

Farazmand, A. (1995). Religion and politics in contemporary Iran: Shia radicalism, revolution, and national character. International Journal on Group Rights, 3(3), 227-257.

Faul, F., Erdfelder, E., Buchner, A., Lang, A. G. (2009). Statistical power analyses using G*Power 3.1: Tests for correlation and regression analyses. Behavior Research Methods, $41,1149-1160$.

Fischer, A. H., \& Manstead, A. S. R. (2008). The social function of emotions. In M. Lewis, J. Haviland-Jones, \& L. F. Barrett (Eds.), Handbook of emotions (Vol. 3, pp. 456-470). New York, NY: Guilford

Foster, S. (2020). Relational aggression in romantic relationships: How do honor-endorsing women traverse conflict with their partners? [Doctoral dissertation, University of Oklahoma]. https://shareok.org/handle/11244/324162

Frijda, N.H., \& Mesquita, B. (1994). The social roles and functions of emotions. In S. Kitayama \& H.R. Markus (Eds.), Emotion and culture (pp. 51-88). Washington, DC: American Psychological Association.

Greenaway, K. H., \& Kalokerinos, E. K. (2019). The intersection of goals to experience and express emotion. Emotion Review, 11(1), 50-62.

Grewal, Z. (2009). Death by culture? How not to talk about Islam and domestic violence. Institute for Social Policy and Understanding. Retrieved from 
https://www.ispu.org/death-by-culture-how-not-to-talk-about-islam-and-domesticviolence/

Gross, J. J., \& John, O. P. (1998). Mapping the domain of expressivity: Multimethod evidence for a hierarchical model. Journal of Personality and Social Psychology, 74(1), 170-191.

Guerra, V. M., Giner-Sorolla, R., \& Vasiljevic, M. (2013). The importance of honor concerns across eight countries. Group Processes \& Intergroup Relations, 16(3), 298-318.

Guerrero, L. K., \& Andersen, P. A. (1998). The experience and expression of romantic jealousy. In P. A. Andersen \& L. K. Guerrero (Eds.), The handbook of communication and emotion: Research, theory, applications, and contexts (pp. 155-188). San Diego, CA: Academic Press.

Guest, G., Bunce, A., \& Johnson, L. (2006). How many interviews are enough? An experiment with data saturation and variability. Field Methods, 18(1), 59-82.

Gul, P., Cross, S. E., \& Uskul, A. K. (2021). Implications of culture of honor theory and research for practitioners and prevention researchers. American Psychologist, 76(3), 502-515.

Günsoy, C., Cross, S. E., Uskul, A. K., \& Gercek-Swing, B. (2020a). The role of culture in appraisals, emotions and helplessness in response to threats. International Journal of Psychology, 55(3), 472-477.

Günsoy, C., Joo, M., Cross, S. E., Uskul, A. K., Gul, P., Wasti, S. A., ... \& Yegin, A. (2020b). The influence of honor threats on goal delay and goal derailment: A comparison of Turkey, Southern US, and Northern US. Journal of Experimental Social Psychology, 88, 103974.

Haidt, J., Koller, S. H., \& Dias, M. G. (1993). Affect, culture, and morality, or is it wrong to eat your dog? Journal of Personality and Social Psychology, 65(4), 613-628. 
Hansen, N., \& Sassenberg, K. (2011). Exploring the self-directed anger of the stigmatized: The interplay between perceived legitimacy and social identification. Group Processes \& Intergroup Relations, 14(6), 807-818.

Hassan, R. (1995). Women in Islam: Quranic ideals versus Muslim realities. Planned Parenthood Challenges, 2, 5-9.

Hassanzadeh, P. (2020). From nomos to Iranian namoos. RadioZamaneh. https://www.radiozamaneh.com/509163

Haven, T., \& Van Grootel, D. L. (2019). Preregistering qualitative research. Accountability in Research, 26(3), 229-244.

Helkama, K., Verkasalo, M., Myyry, L., Silfver, M., Niit, T., Manganelli, A.-M., \& Stetsenko, A. (2013). Honor as a value in Finland, Estonia, Italy, Russia, and Switzerland. Group Processes \& Intergroup Relations, 16(3), 279-297.

Henrich, J., Heine, S. J., \& Norenzayan, A. (2010). The weirdest people in the world?. Behavioral and Brain Sciences, 33(2-3), 61-83.

Herfehdoust, M., Falsafinejad, M., Delavar, A., Sohrabi, F., \& Tamimdari, A. (2015). Extracting the personality factors based on lexical study of the Persian language. Educational Measurement, 6, 83-112.

Hoemann, K., Gendron, M., \& Barrett, L. F. (2022). Assessing the Power of Words to Facilitate Emotion Category Learning. Affective Science. Advance online publication. https://doi.org/10.1007/s42761-021-00084-4

Hupka, R.B. (1991). The motive for the arousal of romantic jealousy: Its cultural origin. In P. Salovey (Ed.), The psychology of jealousy and envy (pp. 252-270). New York: Guilford Press. 
IJzerman, H., van Dijk, W. W., \& Gallucci, M. (2007). A bumpy train ride: A field experiment on insult, honor, and emotional reactions. Emotion, 7(4), 869-875.

Iqbal, A. (2012, August, 4), They do not have a word for "gheirat" in English. Dawn. https://www.dawn.com/news/739736/they-do-not-have-a-word-for-ghairat-in-english

Izard, C. E. (2010). The many meanings/aspects of emotion: Definitions, functions, activation, and regulation. Emotion Review, 2, 363-370.

Jabbari, M., Shahidi, S., Mazaheri, M., \& Panaghi, L. (2021). Character strengths of Iranian adolescents. Quarterly of Applied Psychology,15(3), 355-380.

Jafari, H. (2017, June 18). Infographic: State of Internet \& social media usage in Iran. TechRasa. http://techrasa.com/2017/06/18/infographic-state-internet-social-media-usageiran/

Jamshed, S. (2014). Qualitative research method-interviewing and observation. Journal of Basic and Clinical Pharmacy, 5(4), 87-88.

Kamans, E., Gordijn, E. H., Oldenhuis, H., \& Otten, S. (2009). What I think you see is what you get: Influence of prejudice on assimilation to negative meta-stereotypes among Dutch Moroccan teenagers. European Journal of Social Psychology, 39, 842-851.

Keddie, N. R. (1980). Iran: Change in Islam; Islam and Change. International Journal of Middle East Studies, 11(4), 527-542.

Keltner, D., \& Haidt, J. (2003). Approaching awe, a moral, spiritual, and aesthetic emotion. Cognition and Emotion, 17(2), 297-314.

Keltner, D., \& Haidt, J. (1999). Social functions of emotions at four levels of analysis. Cognition \& Emotion, 13(5), 505-521.

Khezr-Heidari, F. (2018, March 29). Are women men's "namoos,” and is "gheirat" a necessity 
for manhood? Radio Farda.

https://www.radiofarda.com/a/taboo-e71-on-ghayrah/29134126.html

Khurshid, A. (2012). A transnational community of Pakistani Muslim women: Narratives of rights, honor, and wisdom in a women's education project. Anthropology \& Education Quarterly, 43(3), 235-252.

Kommattam, P., Jonas, K. J., \& Fischer, A. H. (2017). We are sorry, they don’t care:

Misinterpretation of facial embarrassment displays in Arab-White intergroup contexts. Emotion, 17(4), 658-668.

Krauss, S. E. (2005). Research paradigms and meaning making: A primer. The qualitative Report, 10(4), 758-770.

Lakens, D. (2013). Calculating and reporting effect sizes to facilitate cumulative science: a practical primer for t-tests and ANOVAs. Frontiers in psychology, 4(863).

Lange, J., \& Zickfeld, J. H. (2021). Emotions as overlapping causal networks of emotion components: Implications and methodological approaches. Emotion Review, 13(2), 157167.

Levitt, H. M. (2021). Qualitative generalization, not to the population but to the phenomenon: Reconceptualizing variation in qualitative research. Qualitative Psychology, 8(1), 95110.

Levitt, H. M., Bamberg, M., Creswell, J. W., Frost, D. M., Josselson, R., \& Suárez-Orozco, C. (2018). Journal article reporting standards for qualitative primary, qualitative metaanalytic, and mixed methods research in psychology: The APA Publications and Communications Board task force report. American Psychologist, 73(1), 26-46.

Levy, R. I. (1984). The emotions in comparative perspective. In Scherer, K.R. \& Ekman, P. 
(Eds.). Approaches to emotion. pp. 397-412. New Jersey: Hillsdale.

Leung, A. K. Y., \& Cohen, D. (2011). Within-and between-culture variation: individual differences and the cultural logics of honor, face, and dignity cultures. Journal of Personality and Social Psychology, 100(3), 507-526.

Lowe, A., Norris, A. C., Farris, A. J., \& Babbage, D. R. (2018). Quantifying thematic saturation in qualitative data analysis. Field Methods, 30(3). 191-207.

Maitner, A. T., Mackie, D. M., Pauketat, J. V., \& Smith, E. R. (2017). The impact of culture and identity on emotional reactions to insults. Journal of Cross-Cultural Psychology, 48(6), 892-913.

Maleki, A \& Arab, P. (2020). Iranians' attitudes toward religion: A 2020 survey report. The Netherlands: Group for Analyzing and Measuring Attitudes in Iran (GAMAAN). Available at: https://gamaan.org/wp-content/uploads/2020/09/GAMAAN-Iran-ReligionSurvey-2020-English.pdf

Malik, H. (2018). Media, gender and domestic relations in post-Saddam Iraq [Doctoral dissertation, Keele University]. https://eprints.keele.ac.uk/5102/1/MalikPhD2018.pdf

Markus, H. R. (2016). What moves people to action? Culture and motivation. Current Opinion in Psychology, 8, 161-166.

Matsumoto, D., Kudoh, T., Scherer, K., \& Wallbott, H. (1988). Antecedents of and reactions to emotions in the United States and Japan. Journal of Cross-Cultural Psychology, 19(3), 267-286.

Matusitz, J. (2012). Relationship between knowledge, stereotyping, and prejudice in interethnic communication. Revista de Turismo y Patrimonio Cultural, 10, 89-98. 
Mayeda, D. T., \& Vijaykumar, R. (2016). A review of the literature on honor-based violence. Sociology Compass, 10(5), 353-363.

Mazaheri, M. (2016). Ma'refat-e-nafs [Knowledge of the self]. Isfahan, Iran: Al-Zahra Publications.

Medin, D., Ojalehto, B., Marin, A., \& Bang, M. (2017). Systems of (non-) diversity. Nature Human Behaviour, 1(5), Article 0088.

Merriam, S. B., \& Tisdell, E. J. (2015). Qualitative research: A guide to design and implementation ( $4^{\text {th }}$ ed.). San Francisco, CA: John Wiley \& Sons.

Mesquita, B., \& Boiger, M. (2014). Emotions in context: A sociodynamic model of emotions. Emotion Review, 6(4), 298-302.

Migration to Europe in charts. (2018, September 11). BBC. https://www.bbc.com/news/worldeurope-44660699

Miyake, K., Chen, S.-J., \& Campos, J. J. (1985). Infant temperament, mother's mode of interaction, and attachment in Japan: An interim report. Monographs of the Society for Research in Child Development, 50(1/2), 276-297.

Moors, A. (2009). Theories of emotion causation: A review. Cognition \& Emotion, 23, 625-662.

Moors, A., Ellsworth, P. C., Scherer, K. R., \& Frijda, N. H. (2013). Appraisal theories of emotion: State of the art and future development. Emotion Review, 5(2), 119-124.

Moradi, S. (2020). Languages of Iran: Overview and critical assessment. In S. Brunn \& R. Kehrein (Eds.), Handbook of the changing world language map (pp. 1171-1202). Springer. https://doi.org/10.1007/978-3-030-02438-3_137

Moradinasab, H. (2021). The importance of Mahramiat in the traditional houses of Iran based 
on the Islamic teachings regarding territory and privacy. Housing and Rural Environment, 39(172), 47-60.

Mortazavi S., Poelker, K. E. (2017) Women in Iran. In: Brown C., Gielen U., Gibbons J., Kuriansky J. (eds) Women's Evolving Lives (pp. 73-90). Cham: Springer International Publishing.

Motahhari, M. (1968). Mas'ale-ye Hijab. [The question of hijab]. Qom, Iran: Sadra Publication. "Mousavi's delusion: Egypt's revolution, the extension of the green movement". (2011, January, 30). Teribon. https://www.teribon.ir/archives/38460

Nisbett, R. E., \& Cohen, D. (1996). Culture of honor: The psychology of violence in the South. Boulder, CO: Westview Press.

Noori, M. (1987). Mustadrak al-wasa'il. Al-Bayt Institute.

Noori Ala, E. (2013). The consequences of "namoosifying” affairs. IranGlobal. https://iranglobal.info/node/15336

Norman, G. (2017). Generalization and the qualitative-quantitative debate. Advances in Health Sciences Education, 22(5), 1051-1055

Novin, S., \& Oyserman, D. (2016). Honor as cultural mindset: Activated honor mindset affects subsequent judgment and attention in mindset-congruent ways. Frontiers in Psychology, 7, Article 1921.

Nowak, A., Gelfand, M. J., Borkowski, W., Cohen, D., \& Hernandez, I. (2016). The evolutionary basis of honor cultures. Psychological Science, 27(1), 12-24.

Öner, S. (2018). Namus: woman as “translation”. Trakya Üniversitesi Edebiyat Fakültesi Dergisi, 8(15), 110-118.

Osterman, L. L., \& Brown, R. P. (2011). Culture of honor and violence against the self. 
Personality and Social Psychology Bulletin, 37, 1611-1623.

Palinkas, L. A., Horwitz, S. M., Green, C. A., Wisdom, J. P., Duan, N., \& Hoagwood, K. (2015). Purposeful sampling for qualitative data collection and analysis in mixed method implementation research. Administration and Policy in Mental Health and Mental Health Services Research, 42(5), 533-544.

Park, N., Peterson, C., Seligman, M. E. P. (2006). Character strengths in fifty-four nations and the fifty US states. The Journal of Positive Psychology, 1, 118-129.

Patton, M. Q. (1990). Qualitative evaluation and research methods. London: SAGE Publications.

Pew Research Center (2009). "Mapping The Global Muslim Population. A Report on the Size and Distribution of the World's Muslim Population.” Pew Research Center: Washington, D.C.

Pfeiffer, S. M., \& Wong, P. T. (1989). Multidimensional jealousy. Journal of Social and Personal Relationships, 6(2), 181-196.

Pitt-Rivers, J. (1965). Honour and social status. In J. Peristiany (Ed.), Honour and shame (pp. 19-78). London, England: Weidenfeld and Nicholson.

Radecki-Bush, C., Farrell, A. D., \& Bush, J. P. (1993). Predicting jealous responses: The influence of adult attachment and depression on threat appraisal. Journal of Social and Personal Relationships, 10, 569-588.

Radford, J. (2019, June 17). Key findings about US immigrants. Pew Research Center. https://www.pewresearch.org/fact-tank/2019/06/17/key-findings-about-u-s-immigrants/ Rodriguez Mosquera, P. M. (2016). On the importance of family, morality, masculine and 
feminine honor for theory and research. Social and Personality Psychology Compass, 10/8, 431-443.

Rodriguez Mosquera, P. M. (2018). Cultural concerns: How valuing social-image shapes social emotion. European Review of Social Psychology, 29(1), 1-37.

Rodriguez Mosquera, P. M., Fischer, A. H., Manstead, A. S., \& Zaalberg, R. (2008). Attack, disapproval, or withdrawal? The role of honour in anger and shame responses to being insulted. Cognition and Emotion, 22(8), 1471-1498.

Rodriguez Mosquera, P. M., Manstead, A. S. R., \& Fischer, A. H. (2002). The role of honor concerns in emotional reactions to offenses. Cognition and Emotion, 16(1), 143-163.

Rosch, E. (1975). Cognitive representations of semantic categories. Journal of Experimental Psychology: General, 104, 192-233.

Roseman, I. J., Wiest, C., \& Swartz, T. S. (1994). Phenomenology, behaviors, and goals differentiate discrete emotions. Journal of Personality and Social Psychology, 67(2), 206-221.

Rozin, P. (2001). Social psychology and science: Some lessons from Solomon Asch. Personality and Social Psychology Review, 5(1), 2-14.

Russell, J. A. (1991a). Culture and the categorization of emotions. Psychological Bulletin, 110(3), 426-450.

Russell, J. A. (1991b). In defense of a prototype approach to emotion concepts. Journal of Personality and Social Psychology, 60(1), 37-47.

Russell, J. A., \& Fehr, B. (1994). Fuzzy concepts in a fuzzy hierarchy: Varieties of anger. Journal of Personality and Social Psychology, 67(2), 186-205.

Said, E. W. (1991). Orientalism: Western conceptions of the Orient. Penguin, London (first 
published 1978).

Salari Rad, M., Martingano, A. J., \& Ginges, J. (2018). Toward a psychology of Homo Sapiens: Making psychological science more representative of the human population. Proceedings of the National Academy of Sciences, 115(45), 11401-11405.

Saucier, G., \& Iurino, K. (2020). High-dimensionality personality structure in the natural language: Further analyses of classic sets of English-language trait-adjectives. Journal of Personality and Social Psychology, 119(5), 1188-1219.

Saunders, B., Sim, J., Kingstone, T., Baker, S., Waterfield, J., Bartlam, B., ... \& Jinks, C. (2018). Saturation in qualitative research: exploring its conceptualization and operationalization. Quality \& Quantity, 52(4), 1893-1907.

Scheel, A., Tiokhin, L., Isager, P., Lakens, D. (2021). Why hypothesis testers should spend less time testing. Perspectives on Psychological Science. 16(4) $744-755$

Scherer, K. R. (1984). Emotion as a multicomponent process: A model and some cross-cultural data. Review of Personality and Social Psychology, 5, 37-63.

Scherer, K. R. (2005). What are emotions? And how can they be measured? Social Sciences Information, 44, 695-729.

Scherer, K. R. (2009). The dynamic architecture of emotion: Evidence for the component process model. Cognition and Emotion, 23(7), 1307-1351.

Schneider, J. (1971). Of vigilance and virgins: Honor, shame and access to resources in Mediterranean societies. Ethnology, 10(1), 1-24.

Schönbrodt, F. D., \& Perugini, M. (2013). At what sample size do correlations stabilize? Journal of Research in Personality, 47(5), 609-612.

Schoonenboom, J., \& Johnson, R. B. (2017). How to construct a mixed methods research design. 
Kölner Zeitschrift für Soziologie und Sozialpsychologie, 69(2), 107-131.

Shackelford, T. K. (2005). An evolutionary psychological perspective on cultures of honor. Evolutionary Psychology, 3(1), 381-391.

Shahabi, M., \& Etaf, V. (2014). A phenomenological study on the male concern about female objectification and self-objectification. Iranian Journal of Sociology, 15(2), 126-152.

Shaheed, F. (1986). The cultural articulation of patriarchy: legal systems, Islam and women. South Asia Bulletin, 6(1), 38-44.

Sharpsteen, D. J. (1993). Romantic jealousy as an emotion concept: A prototype analysis. Journal of Social and Personal Relationships, 10, 69-82.

Shaver, P., Schwartz, J., Kirson, D., \& O'Connor, C. (1987). Emotion knowledge: Further exploration of a prototype approach. Journal of Personality and Social Psychology, 52, $1061-1086$.

Shier, A., \& Shor, E. (2016). "Shades of Foreign Evil" "Honor Killings" and "Family Murders" in the Canadian Press. Violence Against Women, 22(10), 1163-1188.

Shirazi, N. M. (2004). Akhlagh dar Ghor'an. [Ethics in the Qur'an]. Qom, Iran: Imam Ali Pub.

Shokri, O., Goudarzi, A., \& Sharifi, M. (2014). Testing for factorial invariance of the Farsi version of the Positive and Negative Affect Schedule across sexes. Contemporary Psychology, Biannual Journal of the Iranian Psychological Association, 9(1), 83-94.

Shweder, R. A. (1982). Beyond self-constructed knowledge: The study of culture and morality. Merrill-Palmer Quarterly, 41-69.

Tabatabaei, F. (n.d.). Signs of gheirat in men, gheirat or a kind of problem?. Honareh Zendegi. https://honarehzendegi.com/fa/Signs-of-zeal-in-men

Smaldino, P. E., Lukaszewski, A., von Rueden, C., \& Gurven, M. (2019). Niche diversity can 
explain cross-cultural differences in personality structure. Nature Human Behaviour, 3(12), 1276-1283.

Snarey, J. R. (1985). Cross-cultural universality of social-moral development: a critical review of Kohlbergian research. Psychological Bulletin, 97(2), 202.

Soto, C. J., \& John, O. P. (2017). The next Big Five Inventory (BFI-2): Developing and assessing a hierarchical model with 15 facets to enhance bandwidth, fidelity, and predictive power. Journal of Personality and Social Psychology, 113(1), 117-143.

Smith, P. B., Easterbrook, M. J., Koc, Y., Lun, V. M. C., Papastylianou, D., Grigoryan, L., ... \& Chobthamkit, P. (2021). Is an emphasis on dignity, honor and face more an attribute of individuals or of cultural groups? Cross-Cultural Research, 55(2-3), 95-126.

Sommers, T. (2018). Why honor matters. New York: Basic Books.

Srivastava, S. (2018). Sound Inference in Complicated Research: A MultiStrategy Approach. PsyArXiv. https://doi.org/10.31234/osf.io/bwr48

Srivastava, S. (2020). Personality structure: Who cares? European Journal of Personality, 34(4), $550-551$.

Stausberg, M., Vevaina, Y. S.-D., \& Tessmann, A. (Eds.). (2015). The wiley Blackwell companion to Zoroastrianism, John Wiley \& Sons, Ltd.

Tabatabaei, M. H. (1976). Tafsir al-Mizan. [The balance in interpretation of Quran] 3rd ed., Tehran, Iran: House of Islamic Books.

Tackman, A. M., \& Srivastava, S. (2016). Social responses to expressive suppression: The role of personality judgments. Journal of Personality and Social Psychology, 110(4), 574591.

Takahashi, K. (1990). Are the key assumptions of the "strange situation" procedure universal? A 
view from Japanese research. Human Development, 33(1), 23-30.

Tashakkori, A., \& Teddlie, C. (1998). Mixed methodology: Combining qualitative and quantitative approaches (Vol. 46). Thousand Oaks, CA: Sage.

Tavakoli-Targhi, M. (1999). Contested Memories of Pre-Islamic Iran. The Medieval History Journal, 2(2), 245-275.

Tawasil, A. (2015). Towards the Ideal Revolutionary Shi'i Woman: The Howzevi (Seminarian), the Requisites of Marriage and Islamic Education in Iran, Hawwa, 13(1), 99-126.

Taylor, A. (2016, May 25). In Switzerland, Muslim schoolchildren who refuse to shake their teacher's hand may be fined \$5,000. The Washington Post. https://www.washingtonpost.com/news/worldviews/wp/2016/05/25/in-switzerlandmuslim-schoolchildren-who-refuse-to-shake-their-teachers-hand-may-be-fined-5000/ Terracciano, A., Abdel-Khalek, A. M., Adam, N., Adamovová, L., Ahn, C. K., Ahn, H. N., ... \& Avia, M. D. (2005). National character does not reflect mean personality trait levels in 49 cultures. Science, 310(5745), 96-100.

Thalmayer, A. G., Job, S., Shino, E. N., Robinson, S. L., \& Saucier, G. (2021). ‡Ūsigu: A mixedmethod lexical study of character description in Khoekhoegowab. Journal of Personality and Social Psychology, 121(6), 1258-1283.

The APA Presidential Task Force on Immigration. (2013). Crossroads: The psychology of immigration in the new century. Journal of Latina/o Psychology, 1(3), 133-148.

"The gheirat and courage of the Iranian nation won the war". (2013, September, 22). Iqna. https://iqna.ir/005PzS

"The martyr Fahmideh moved for the country's security and namoos". (2021, October, 29). DefaPress. https://defapress.ir/486137 
The World Bank Data (2020). GDP (current US\$) [Data file]. Retrieved from https://api.worldbank.org/v2/en/indicator/NY.GDP.PCAP.CD?downloadformat=csv

Thrasher, J., \& Handfield, T. (2018). Honor and violence. Human Nature, 29(4), 371-389.

Torabifard, H. R. (2020, February 8). "The gheirat of army men won the Islamic revolution". IRNA. https://www.irna.ir/news/83665437/

Tsai, J. L., Knutson, B., \& Fung, H. H. (2006). Cultural variation in affect valuation. Journal of Personality and Social Psychology, 90(2), 288-307.

Uskul, A. K., Cross, S. E., Alözkan, C., Gercek-Swing, B., Ataca, B., Günsoy, C., \& Sunbay, Z. (2014). Emotional responses to honour situations in Turkey and the northern USA. Cognition and Emotion, 28(6), 1057-1075.

Uskul, A. K., Cross, S. E., Günsoy, C., \& Gul, P. (2019). Cultures of honor. In Kitayama, S., \& Cohen, D., eds. (pp. 793-821). Handbook of Cultural Psychology. The Guilford Press, New York.

Uskul, A. K., Cross, S. E., Sunbay, Z., Gercek-Swing, B., \& Ataca, B. (2012). Honor bound: The cultural construction of honor in Turkey and the Northern United States. Journal of Cross-Cultural Psychology, 43(7), 1131-1151.

Vandello, J. A., \& Cohen, D. (2004). When believing is seeing: Sustaining norms of violence in cultures of honor. In M. Schaller \& C. S. Crandall (Eds.), The psychological foundations of culture (pp. 281-304). Lawrence Erlbaum Associates Publishers.

Veillard, N. (2017). WEIRD sampling in cross-cultural psychology, should it not be less WEIRD and more representative? [Unpublished master's thesis]. Leiden University.

Verkuyten, M., \& Zaremba, K. (2005). Interethnic relations in a changing political context. Social Psychology Quarterly, 68, 375-386. 
Wallbot, H., \& Scherer, K. (1986). The antecedents of emotional experiences. In K. Scherer, H. Wallbott, \& A. Summerfield (Eds.), Experiencing emotion: A cross-cultural study (pp. 69-83). Cambridge: Cambridge University Press.

Watson, D., \& Clark, L. A. (1994). The PANAS-X: Manual for the Positive and Negative Affect Schedule-Expanded Form. Department of Psychological \& Brain Sciences Publications, University of Iowa, Iowa City, IA.

White, G. L., \& Mullen, P. E. (1989). Jealousy: Theory, research, and clinical strategies. New York, US: Guilford Press.

White, C. J. M., Muthukrishna, M., Norenzayan, A. (2021). Cultural similarity among coreligionists within and between countries. Proceedings of the National Academy of Science, 118 (37).

Wierzbicka, A. (1986). Human emotions: Universal or culture-specific? American Anthropologist, 88(3), 584-594.

Wierzbicka, A. (1997). Understanding cultures through their key words. New York \& Oxford: Oxford University Press.

Windfuhr, G. L. (1991, December 15). Central Dialects. Encyclopaedia Iranica. Retrieved February 13, 2022, from https://iranicaonline.org/articles/central-dialects

Wu, K., \& Dunning, D. A. (2019, October 17). Hypocognitive mind: How lack of conceptual knowledge confines what people see and remember. https://doi.org/10.31234/osf.io/29ryz 